\title{
Image Segmentation and Co-registration in an Intra Operative Multimodal Imaging System (IMIS)
}

\author{
A Thesis \\ Presented to \\ the faculty of the School of Engineering and Applied Science \\ University of Virginia
}

\author{
In Partial Fulfillment \\ of the requirements for the degree \\ Master of Science in Electrical Engineering \\ By \\ Anoosha Reddy Papireddy
}

August 2013 


\title{
Approval Sheet
}

This thesis is submitted in partial fulfillment of the requirements for the degree of Master of Science in Electrical Engineering

\author{
Anoosha R Papireddy-Author
}

This thesis has been read and approved by the examining Committee:

Mark B Williams - Thesis Advisor

$\underline{\text { Scott T Acton-Committee Chair }}$

Zongli Lin-Committee Member

Accepted for the School of Engineering and Applied Science:

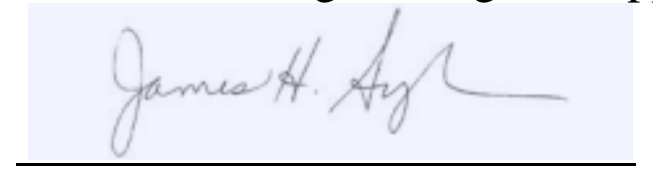

Dean, School of Engineering and Applied Science

August 2013 


\section{ACKNOWLEDGEMENTS}

I would like to acknowledge and thank all those who supported me during the development of this research work. First, I would also like to thank my advisor, Dr Mark Williams for his professional guidance. I am grateful to Dr Acton's encouragement and support throughout the past two years. I would like to thank my colleagues in the VIVA lab and Dr Williams' lab for their assistance and constructive suggestions that made this research work and thesis possible. I am grateful to the support extended by my fellow graduate student Kosta Popovic who has been my mentor. I would also like to thank Dr. Zongli Lin, for serving on my committee and helping to finalize this thesis. Lastly, I would like to thank my family and friends for their unwavering love, support, and trust. 


\begin{abstract}
In this thesis, a new method for segmentation and co-registration of images from multiple modalities is presented. This approach has been tailored to meet the requirements of the Intraoperative Multimodal Imaging System (IMIS) being developed by Dr Mark Williams' lab in the department of Radiology and Medical Imaging at the University of Virginia. The IMIS can be used in a number of applications such as determining the location of primary and secondary carcinomas and sentinel lymph node biopsy. It consists of three imaging modalities- visible, near infrared fluorescence (NIRf), and gamma. The goal of my work is to segment the gamma images and co-register the gamma images with the visible and NIRf images. A series of filtering, binary morphology, edge detection, and active contour based techniques have been used for gamma image segmentation. Image co-registration is achieved with the help of two Attitude and Heading Reference System (AHRS) units.
\end{abstract}

This method has been tested in phantom studies and other laboratory settings and has shown promising results. Plans for small animal imaging and clinical trials in the operation room setting are underway. 


\section{TABLE OF CONTENTS}

ACKNOWLEDGEMENTS ............................................................................... III

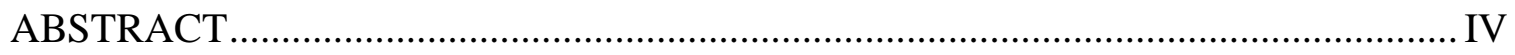

LIST OF ABBREVIATIONS AND SYMBOLS .................................................... X

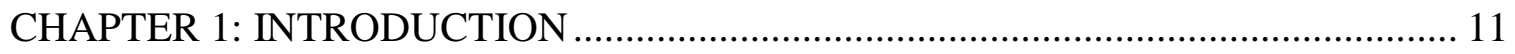

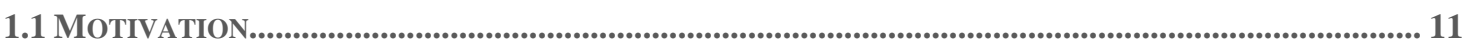

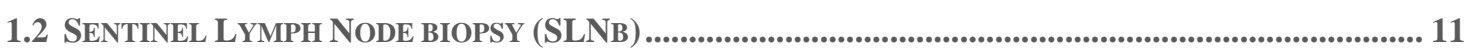

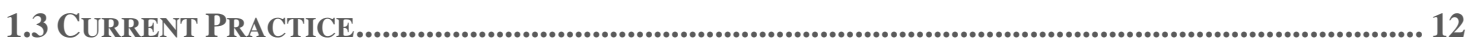

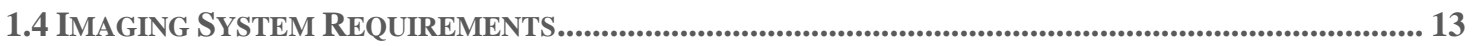

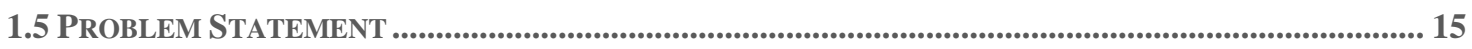

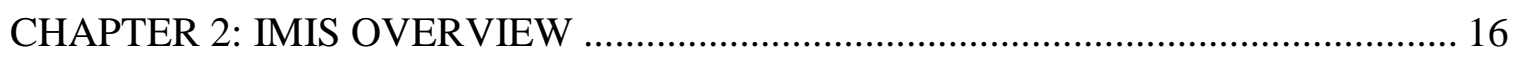

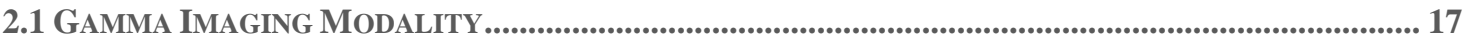

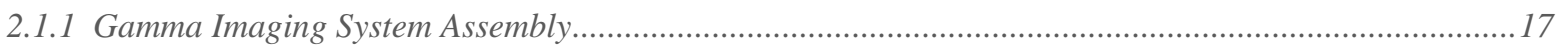

2.1.2 Image Acquisition and Display Software for Gamma Modality ..........................................................19

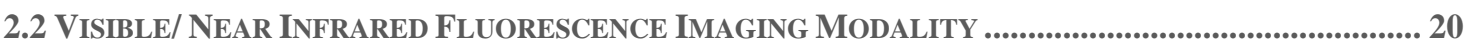

2.2.1 Visible/ Near Infrared Fluorescence Imaging System Assembly ........................................................23

2.2.2 Image Acquisition and Display Software for Visible/NIRf Modalities...............................................25

CHAPTER 3: GAMMA IMAGE SEGMENTATION ................................................ 27

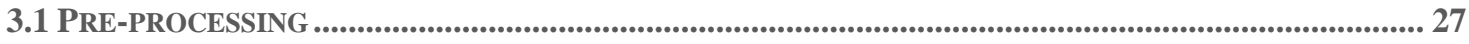

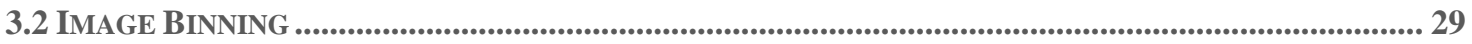

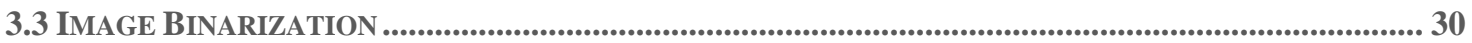

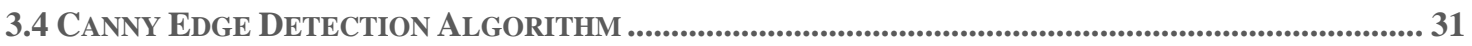

3.5 ACTIVE Contour SEgMENTATION- SNAKES....................................................................................... 33

3.6 Calculating THE NUMber OF GAMMA RAY COUNTS ....................................................................... 35

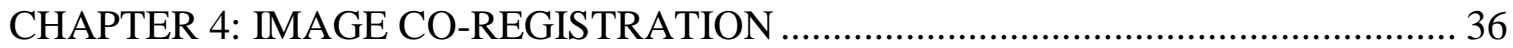

4.1 AligNMENT OF THE TWO CAMERAS .......................................................................................................... 38

4.2 OFFSET CORRECTION.................................................................................................................................... 40

4.3 IMAGE CO-REGISTRATION WALK THOUGH ........................................................................................... 42

CHAPTER 5: EXPERIMENTAL RESULTS ................................................... 45

5.1 REPEATABILITY OF THE GAMMA IMAGE SEGMENTATION ........................................................45

5.2 Calculating THE NUMBer OF COUNTS...................................................................................... 45

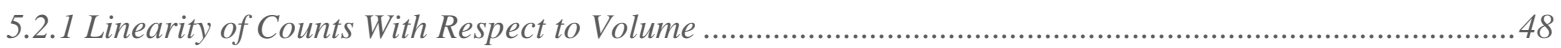

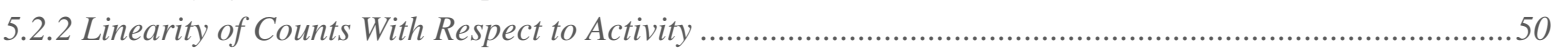

5.3 Repeatability of the Full Process (Segmentation and Co-Registration).................... 51

5.4 COMPARISON OF THE GAMMA IMAgE SEgMENTATION WITH A LOCAL THRESHOLD BASED

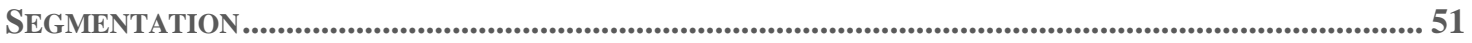

5.5 Sensitivity of the Segmentation Program to Changes in the Target Parameters .... 54 


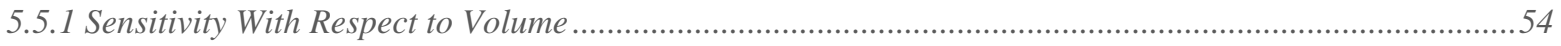

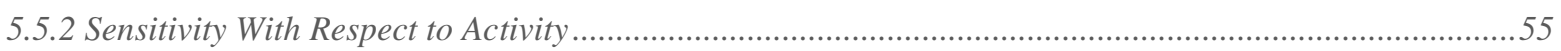

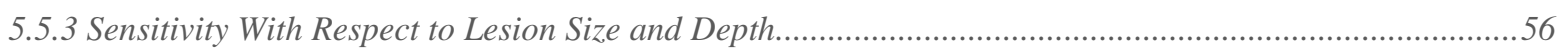

CHAPTER 6: DISCUSSION/CONCLUSION .............................................................. 59

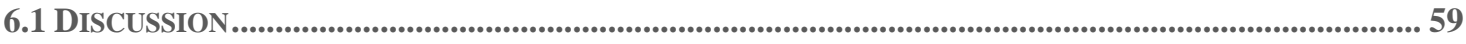

A LITERATURE STUDY WAS DONE TO COMPARE THE RELATIVE ADVANTAGES AND DISADVANTAGES OF USING ONLY NIRF IMAGING AND OF COMBINING GAMMA IMAGING. MOST OF THE CURRENT APPLICATIONS OF NIRF ONLY IMAGING WERE IN FIELDS THAT DO NOT REQUIRE TARGET LOCALIZATION, SUCH AS TISSUE GRAFT QUALITY TESTING AND CERVICAL CANCER SURGERY [18] [19]. ONE OF THE MAIN NIRF SYSTEMS WAS REPORTED BY DEGRAND [20]. IT IS CALLED FLUORESCENCE-ASSISTED RESECTION AND EXPLORATION (FLARE) SYSTEM. THE SYSTEM HAS BEEN USED IN ANIMAL MODEL TRIALS [20, 21, 22, 23, 24] AND HUMAN CLINICAL TRIALS INCLUDING BREAST CANCER SLNB [25, 26], EX-VIVO COLORECTAL SLN MAPPING [24] HEAD AND NECK CANCER SLNB [27] AND CERVICAL CANCER SLN MAPPING [28]. HANDA [29] REPORTED DEVELOPMENT OF A METHOD OF NIRF IMAGING, THAT WAS USED IN INTRAOPERATIVE GRAFT ASSESSMENT AS WELL AS FLUORESCENCE GUIDED SLNB IN ANIMAL MODELS [30]. A COMMERCIAL NIRF IMAGING SYSTEM, NOVADAQ SPY WAS USED IN VARIOUS CLINICAL MEDICAL IMAGING APPLICATIONS, INCLUDING CORONARY AND GRAFT IMAGING [31, 32] ORGAN TRANSPLANTATION [33] AND OTHER CARDIAC FUNCTION ASSESSMENT PROCEDURES [34]. ONE GROUP EXPLORED THE POSSIBILITY OF USING GAMMA AND NIRF IMAGING AS COMPLEMENTARY MODALITIES AND CONCLUDED THAT IT WOULD GIVE BETTER RESULTS THAN NIRF ALONE

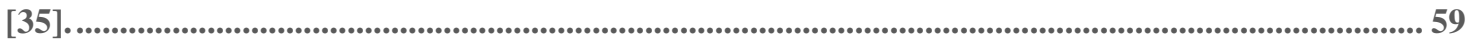

9.2 CONCLUSION........................................................................................................................................................... 63

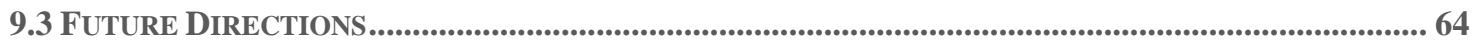




\section{LIST OF TABLES}

TABLE 1: COMPARISON OF THE MEL54 DATA FROM EX-VIVO SLN COUNTS FROM GAMMA CAMERA AND GAMMA PROBE. 48

TABLE 2: COMPARISON OF THE NUMBER OF NODES DETECTED BY EACH SEGMENTATION PROGRAM

TABLE 3: TIME TAKEN BY THE SEGMENTATION PROGRAM …….....................................................6 


\section{LIST OF FIGURES}

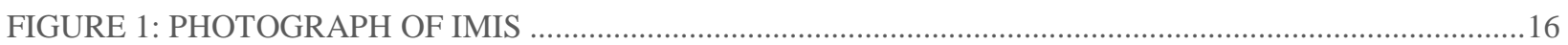

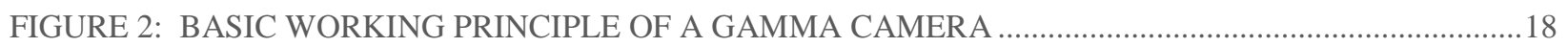

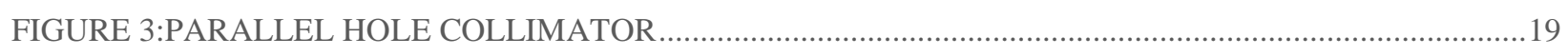

FIGURE 4:BACK OF THE PCB SIPMS INSIDE THE HOUSING WITH CABLES LEADING TO THE

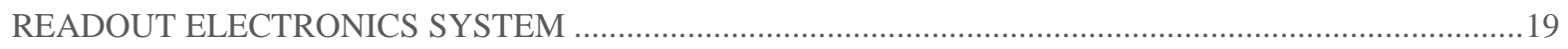

FIGURE 5: SCREENSHOT OF THE GAMMA IMAGE ACQUISITION/DISPLAY SOFTWARE........................20

FIGURE 6: WORKING PRINCIPLE OF FLUORESCENCE..............................................................................21

FIGURE 7: ABSORPTION (BLUE) AND EMISSION (PURPLE) SPECTRA OF INDOCYANINE GREEN (ICG)

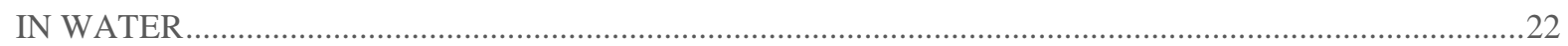

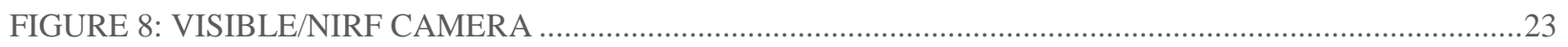

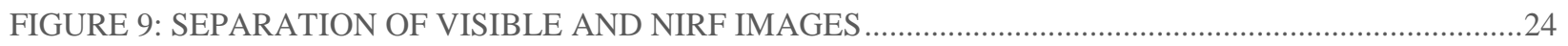

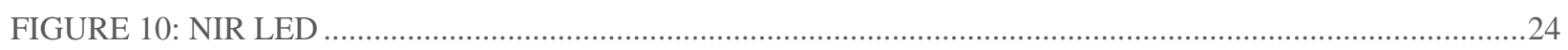

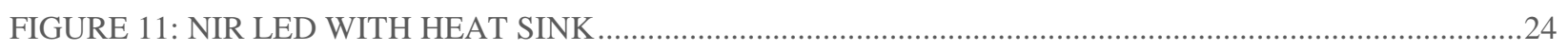

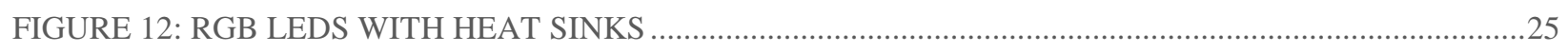

FIGURE 13: SCREENSHOT OF LABVIEW BASED GUI FOR THE ACQUISITION AND DISPLAY OF THE

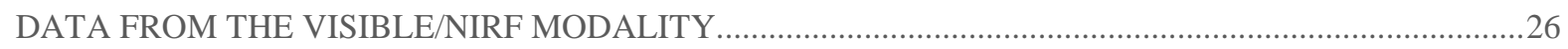

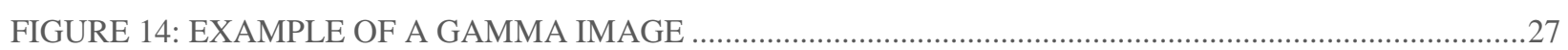

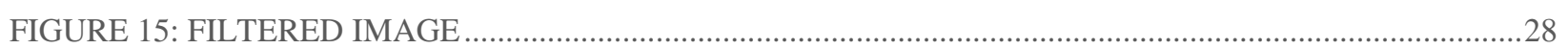

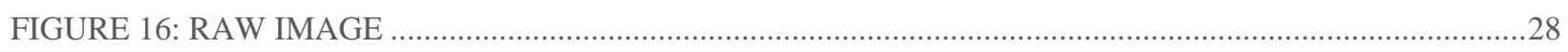

FIGURE 17: EXAMPLES OF BINNING RAW IMAGES (FROM LEFT TO RIGHT 1X, 2X, 3X AND 4X).........29

FIGURE 18: BINNED IMAGE, BINARY IMAGE, BINARY IMAGE- CLEANED (LEFT TO RIGHT) ................30

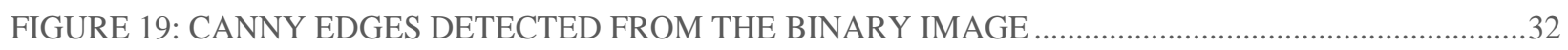

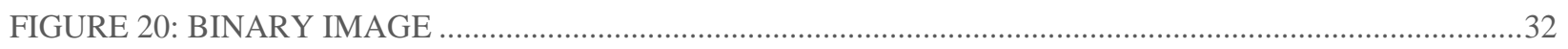

FIGURE 21: FINAL ROI SEGMENTED BY THE SNAKE IS SHOWN IN RED ……………….........................

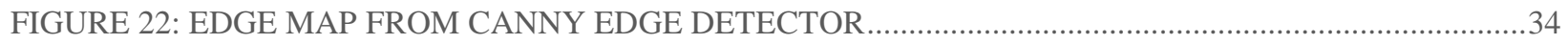

FIGURE 23: IMAGE OF A SEGMENTED NODE WITH THE CORRESPONDING NUMBER OF COUNTS ....35

FIGURE 24: SCREENSHOT OF THE MATLAB GUI DURING SEGMENTATION …..........................................37

FIGURE 25: SCREENSHOT OF THE MATLAB GUI DURING OFFSET CORRECTION ....................................

FIGURE 26:ILLUSTRATION OF THE MISALIGNMENT EFFECT ON THE CO-REGISTRATION ERROR .....38

FIGURE 27: AHRS UNITS MOUNTED ON THE GAMMA CAMERA(LEFT) AND VISIBLE/NIRF

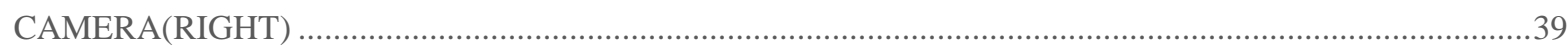

FIGURE 28: GUI SHOWING THE ORIENTATION VECTORS OF THE AHRS UNITS .......................................39

FIGURE 29: OFFSET CORRECTION USING THE LOGO OF THE AHRS UNIT ON THE GAMMA CAMERA

FIGURE 30: PHOTOGRAPH OF THE OPAQUE GELATIN PHANTOM PRIOR TO SURGERY ........................42

FIGURE 31: VIDEO FRAME OF GAMMA CAMERA DURING GAMMA IMAGE ACQUISITION ...................42

FIGURE 32: CONTROL MONITOR SHOWING THE ORIENTATIONS OF THE TWO AHRS UNITS ...............42

FIGURE 33: BINARY IMAGE OF GAMMA CAMERA AHRS LOGO, USED TO CALCULATE ROTATIONAL,

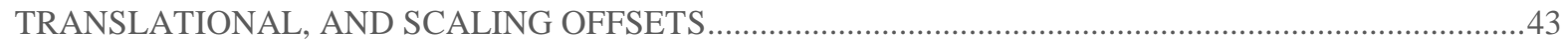

FIGURE 34: ORIGINAL GAMMA IMAGE SHOWING THE TWO TARGETS AND THEIR SEGMENTED

ROIS, BEFORE ROTATIONAL AND TRANSLATIONAL TRANSFORMATION .......................................43

FIGURE 35: VISIBLE FRAME WITH TRANSFORMED ROIS OVERLAID ………………….........................43

FIGURE 36: FUSED VISIBLE/NIRF IMAGE WITH GAMMA ROIS ......................................................................4

FIGURE 37: FLOWCHART OF IMAGE SEGMENTATION AND CO-REGISTRATION PROCESSES...............44

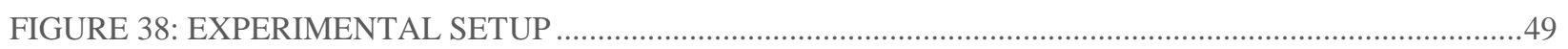


FIGURE 39: GRAPH OF THE NUMBER OF COUNTS WITH RESPECT TO INCREASING VOLUME OF ISOTOPE..... . .49

FIGURE 40: GRAPH OF THE NUMBER OF COUNTS WITH RESPECT TO INCREASING ACTIVITY OF

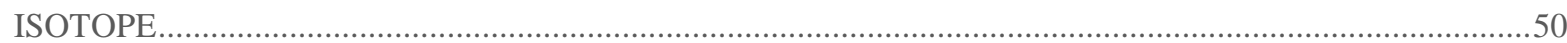

FIGURE 41: LOCAL THRESHOLD BASED SEGMENTATION (LEFT) COMPARED TO CURRENT METHOD

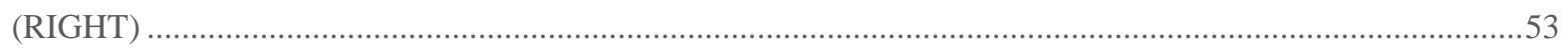

FIGURE 42: GRAPH OF DIAMETER OF TARGETS OF SAME ACTIVITY BUT VARYING VOLUME.........54

FIGURE 43: GRAPH OF THE DIAMETER OF A TARGET WITH RESPECT TO VARYING ACTIVITY.........55

FIGURE 44: SETUP OF THE EXPERIMENT TO MEASURE SENSITIVITY WITH RESPECT TO LESION

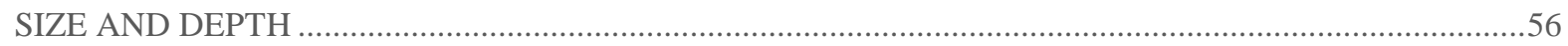

FIGURE 45: GRAPH OF DIAMETER OF LESION IN GAMMA IMAGE WITH RESPECT TO ACTUAL DIAMETER 57

FIGURE 46: GRAPH OF LESION DIAMETER WITH RESPECT TO DEPTH .............................................58

FIGURE 47: TIME TAKEN TO ALIGN THE CAMERAS AND IMAGE BACK OF THE GAMMA CAMERA..60 


\section{LIST OF ABBREVIATIONS AND SYMBOLS}

ADC - Analog to digital converter

APD - Avalanche photodiode

CCD - Charge coupled device

$\mathrm{COG}$ - Center of gravity

DAQ - Data acquisition system

FOV - Field of view

fps - Frames per second

FWHM - Full Width at Half Maximum

GUI - Graphical user interface

HSA - Human serum albumin

ICG - Indocyanine green

$\mathrm{LaBr}_{3}$ - Lanthanum (III) Bromide

LED - Light emitting diodes

MGC - Mobile gamma camera

NIR - Near infrared

NIRf - Near infrared fluorescence

$\mathrm{OR}$ - Operating room

PCB - Printed circuit board

PDE - Photon detection efficiency

PMT - Photomultiplier tube

SBR - Signal to background ratio

SiPM - Silicon photomultiplier

SLN - Sentinel lymph node

SLNB - Sentinel lymph node biopsy

${ }^{99 m}$ Tc - Technetium-99m, a metastable nuclear isomer of Technetium-99, commonly used as a medical radioisotope

${ }^{99 \mathrm{~m}} \mathrm{Tc}-\mathrm{SC}-{ }^{99 \mathrm{~m}} \mathrm{Tc}$ sulfur colloid

WD - Working distance 


\section{CHAPTER 1: INTRODUCTION}

In the past several years, Dr. Mark Williams' laboratory at the University of Virginia has investigated a novel imaging apparatus called the IMIS. Specifically, Dr. Kosta Popovic has endeavored to develop and refine this technology. As such, much of the background relevant to this thesis is derived from his dissertation research (1).

\subsection{Motivation}

Some of the major concerns during the treatment of cancer are the possibility of a local recurrence, false positives, and the need for re-excision of a tumor. It is illustrated by examining the re-excision rates for non-palpable breast lesions, which are reported to be between $20 \%$ and $50 \%$. In melanoma, false negatives are seen in up to $24 \%$ of the cases (2) (3) (4) (5). These occurrences add trauma, cost, and risk to the patient. It is believed that better intra-operative imaging capabilities lower these risks. The goal of this project is to develop one such system.

\subsection{Sentinel Lymph Node biopsy (SLNb)}

A lymph node is a part of the immune system. It is present throughout the body, for example, in the armpit and stomach and linked by lymphatic vessels. Lymph nodes are used to filter or trap foreign particles and play an important role in the proper functioning of the immune system (6). The sentinel lymph node is considered to be the first lymph node, or group of nodes, draining a cancer. It is hypothesized that the sentinel lymph nodes are the target organs which are reached first by metastasizing (spreading from one organ to a non-adjacent organ) cancer cells from 
the tumor (7). Hence, sentinel lymph node biopsy (SLNb) is used to stage certain types of cancer. Similar procedures are used for SLNb and tumor excision. Sometimes they are performed simultaneously. Hence, the surgical guidance system is designed to be useful to SLNb as well as primary carcinoma excision.

\subsection{Current Practice}

SLNb is usually done with the help of blue dye in visual blue dye detection method or with the help of ${ }^{99 \mathrm{~m}} \mathrm{Tc}$ in pre- and intra-operative nuclear imaging. It has been shown nuclear imaging procedures are more successful than the blue dye detection method $(8 ; 9)$. Therefore, this chapter will deal only with the nuclear medicine methods.

There are two major nuclear imaging methods used for SLNb. They are pre-operative planar lymphoscintigraphy and intra-operative gamma probe detection. In Planar lymphoscintigraphy, by injecting ${ }^{99 \mathrm{~m}} \mathrm{Tc}$ and a blue dye is injected into the tumor. Then the location of the sentinel lymph node is determined by recording the path of the blue dye. The pre-operative planar lymphoscintigraphy is sometimes coupled with SPECT/CT to show more accurate information about the exact location of the nodes and lymphatic drainage patterns $(10 ; 11 ; 12)$. This method results in much greater radiation dose to the patient compared to lymphoscintigraphy alone and the equipment is more expensive. Hence is not used regularly and it cannot be used for intra operative imaging.

The intra-operative gamma probe method has been developed to address this need. It is most often used along with the blue dye and with planar pre-operative lymphoscintigraphy. In this procedure, the patient is injected with ${ }^{99 \mathrm{~m}} \mathrm{Tc}$-SC (sulfur colloid). It is done right before the start of the surgery. Then a fixed gamma camera is used to image the patient and the regions of 
interest are marked. This is achieved by recording lymphatic flow and lymph on the images. During the surgery, the surgeon uses a hand-held gamma probe. It is a radiation-detecting device that gives an auditory output. Meter read-outs are additionally displayed to give information about the number of counts detected. A combination of the above information is used to locate ${ }^{99 m}$ Tc-SC within the patient.

There are some major drawbacks associated with this method. First, a fixed gamma camera is needed, which is not feasible to use in the operating room. It also results in loss of post-operative data acquisition to ensure that complete excision cannot be performed. The gamma probe does not provide images to help the surgeon. There are also some practical difficulties for the patient. These include the need for additional scheduling for pre-operative imaging and additional wait time. The following section describes the necessary characteristics of a system to overcome these drawbacks and proposes a novel way of addressing them.

\subsection{Imaging System Requirements}

Surgical guidance is required for both SLNb as described above and for the excision of tumors. A good surgical guidance system should have high sensitivity, good resolution, high accuracy, compact size, and short imaging time. However, there is an inverse relationship between performance in resolution and sensitivity. Imaging time and size vary for different modalities. All these parameters need to be considered in the process of selecting imaging modalities for a particular application. For SLNb or primary carcinoma excision, a surgical guidance system has two main functions: Guiding the surgeon to the location of tumor or SLN and showing the extent of the tissue to be excised. Cancerous tumors can sometimes be non-palpable. Hence, it is important to show the tumor borders accurately and in high resolution to ensure complete 
excision. Any un-excised cancerous tissue could lead to cancer recurrence. Hence, the system should have good sensitivity through several layers of tissue for initially guiding the surgeon to the tumor/SLN and also high resolution once the tumor is in view. It should be able to operate in real time and be compact enough to be used in the operating room.

None of the existing modalities satisfy all of the above requirements. For example, X-ray and MRI modalities provide high sensitivity and resolution but are too bulky. Ultrasound is compact but lacks good resolution. The common drawback of these modalities is that they do not have target specific tracers and cannot provide information on the malignancy of the tumor. Nuclear medicine techniques (PET and SPECT) use targeting agents and offer much better specificity and sensitivity through substantial tissue thickness. They can also be modified for hand-held use in the operating room, but the resolution is poor. Optical imaging techniques using fluorescent agents can provide very good specificity with excellent resolution and compact size, but the sensitivity through thick tissue is poor due to high attenuation.

One way to work around this problem is to combine two or more different modalities, which would together satisfy all the requirements for a particular application. Surgical guidance through intraoperative imaging of lymph nodes and tumors requires imaging through several layers of tissue and targeted imaging. Nuclear imaging satisfies these criteria. However, to compensate for the poor resolution, optical fluorescence imaging is required. The combination of planar gamma scintigraphy and near infrared fluorescence (NIRf) imaging can satisfy all the above imaging requirements. Gamma scintigraphy provides good spatial resolution and high sensitivity through thick biological tissue. This can be used for initial pre-incision tumor or node localization. NIRf modality provides excellent spatial resolution, provided that the target is close to the surface $(1-2 \mathrm{~cm})$. Precise visualization of the node or tumor margins near the surface can 
be achieved once the surgeon is near the target. Both modalities can be used for real-time imaging and can be made compact. The intra-operative multimodal imaging system (IMIS) uses this principle to provide effective surgical guidance.

\subsection{Problem Statement}

The IMIS consists of gamma, visible, and NIRf imaging modalities. In order to maximize the benefits of this system, it is necessary to combine the information from separate modalities into a single image through the process of image co-registration. The surgeon would not only be able to see the hot spots from the gamma image and fluorescence from the NIR image, but also the physical locations of the targets. A combined image would help in precisely determining which tissue has to be excised. However, different modalities inherently have different resolutions and fields of view. In addition, both cameras in the system are free hand in use, which poses significant difficulties. The following chapters describe the process of segmentation and coregistration of different modality images in IMIS in detail. 


\section{CHAPTER 2: IMIS OVERVIEW}

The IMIS combines gamma scintigraphy, near infrared fluorescence (NIRf) and visible modalities to provide both high spatial resolution and high sensitivity through several layers of tissue. The basics of the above modalities and their hardware components have been described in detail in the following sections.

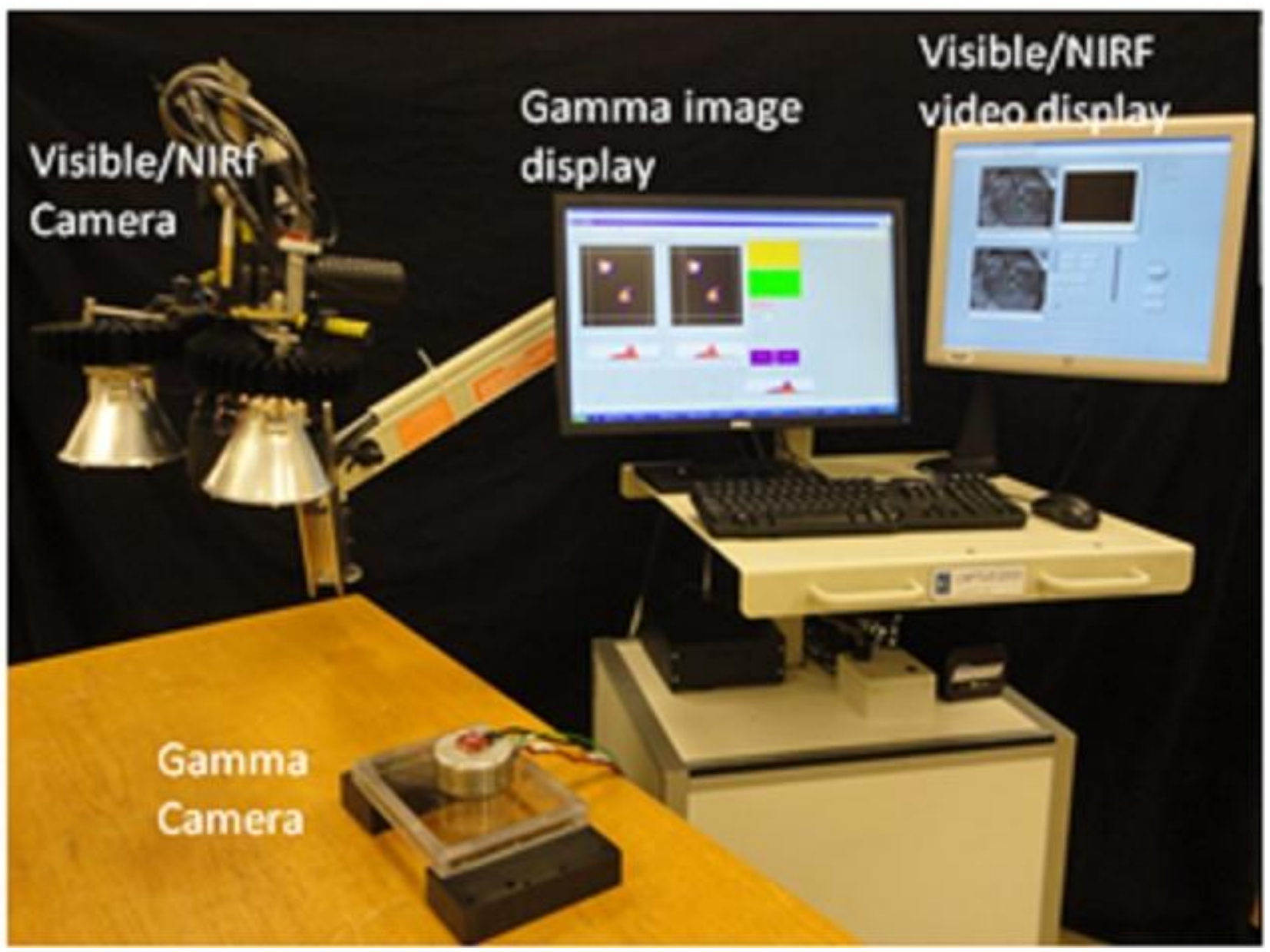

Figure 1: Photograph of IMIS 


\subsection{Gamma Imaging Modality}

Gamma radiation is a type of electromagnetic radiation. It has high frequency and high energy. Gamma rays are usually produced by when atomic nuclei decay from high energy states to lower energy states. When a nucleus emits an $\alpha$ or $\beta$ particle, the daughter nucleus is at a higher energy level. It moves to a lower energy state by emitting energy in the form of a gamma ray. A gamma camera, also called a scintillation camera is used to image gamma radiation to perform

scintigraphy. ${ }^{99 \mathrm{~m}}$ Tc-pertechnetate is used as the targeting agent and is injected into the patient. The distribution of the radiotracer uptake is then imaged by the gamma camera described below.

\subsubsection{Gamma Imaging System Assembly}

The gamma imaging system consists of a camera and a readout system. The camera is capable of detecting gamma rays that are emitted by radioactive tracers injected in the patient. A gamma camera consists of one or more scintillators coupled to an array of photomultiplier tubes that is connected to readout electronics and a computer. The detectors make use of scintillation, a physical process in which the material emits photons as a result of an ionization event in the material. When a gamma ray hits the scintillator, it interacts with and deposits part or all of its energy to an electron, knocking it out from the atom. An electron from the outer shell fills the vacated hole, releasing energy in the form of a characteristic photon that is collected by a photomultiplier tube. When coupled with photomultiplier tubes (PMT), these characteristic photons liberate more electrons through the photoelectric effect. After the amplification process, the magnitude of the charge is sent to the electronic readout equipment. Since the gamma ray emission from a radioactive source is an isotropic process (non-preferential in terms of 
direction), a collimator is required in front of the gamma camera to aid image formation by helping to define the incident direction of the gamma ray and to avoid flooding the gamma camera. The process of detecting emitted photons by a gamma camera is depicted in the figure below.

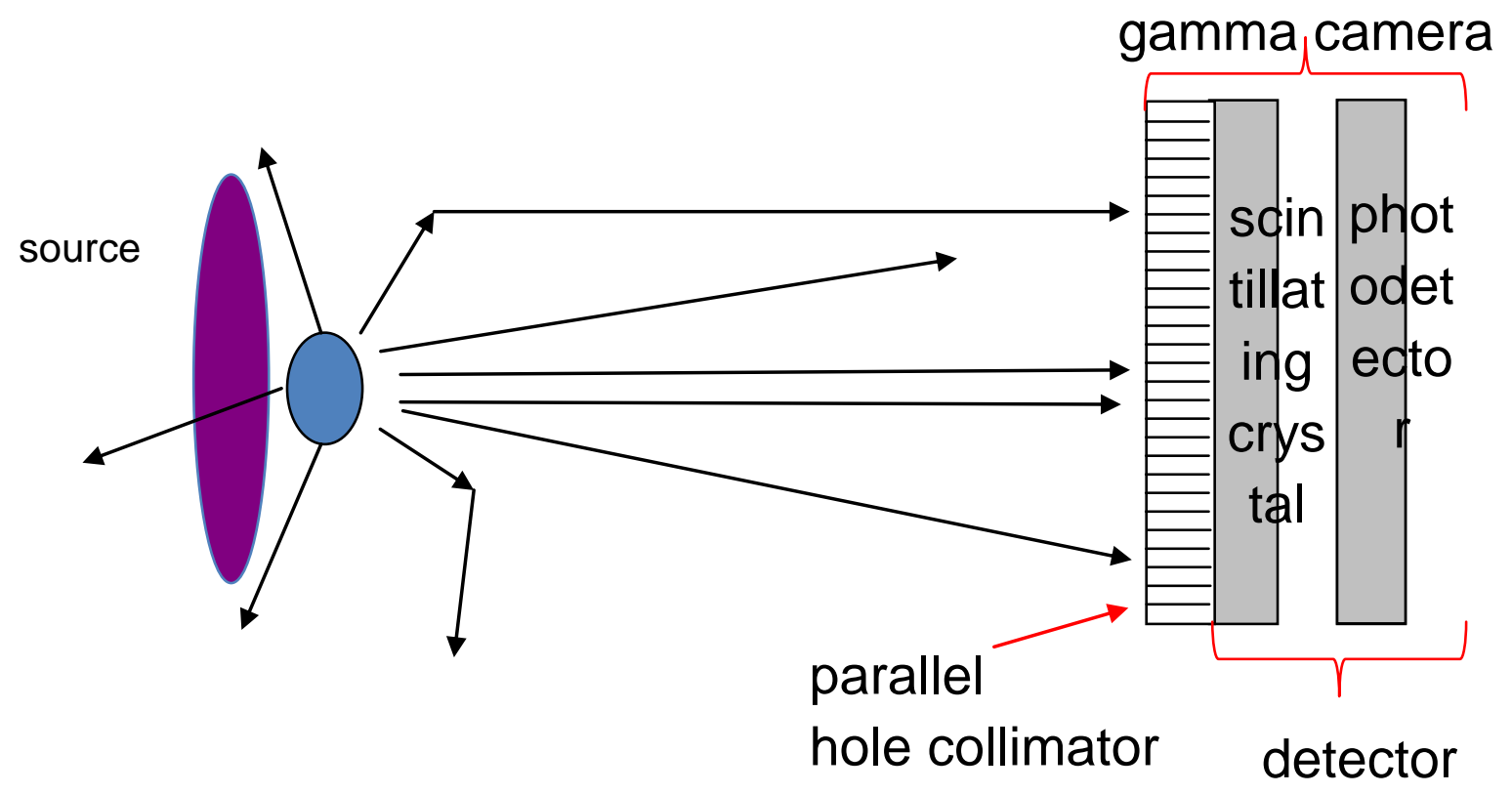

image

Figure 2: BASIC WORKING PRINCIPLE OF A GAMMA CAMERA

For the specific imaging task and considerations in design and size for the gamma camera described in this work, a $\mathrm{LaBr}_{3}: \mathrm{Ce}$ single crystal is used as the scintillator. Silicon Photomultipliers (SiPM) are used instead of conventional PMTs because of their small size and weight. The camera has a custom designed tungsten-polymer composite two-part collimator with an active diameter of $60 \mathrm{~mm}$ and overall diameter of $75 \mathrm{~mm}$. It can be used in either high resolution (two-layer) or high sensitivity (single layer) imaging. 


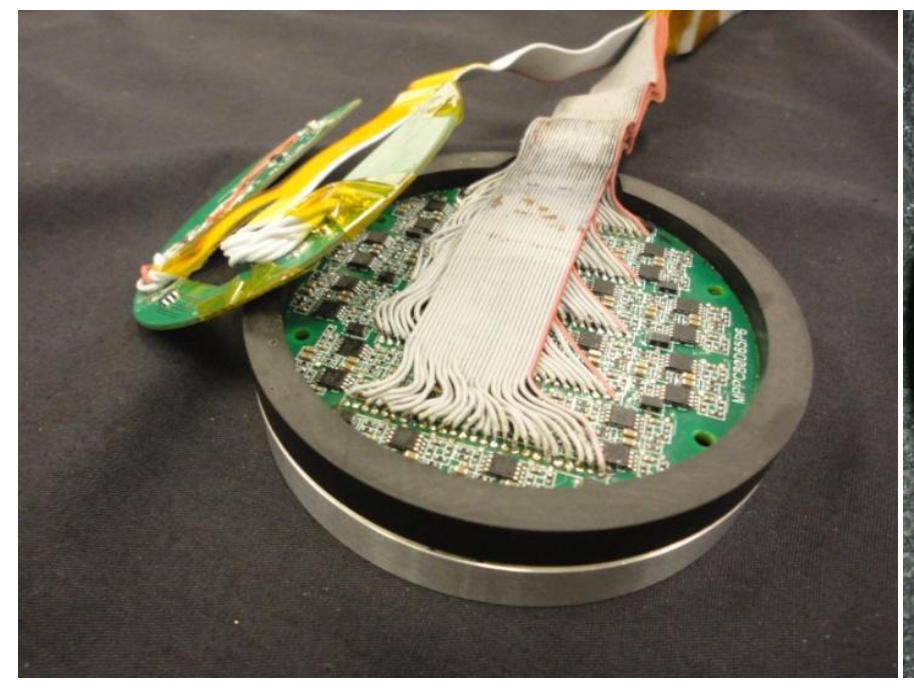

Figure 4:BaCK of the PCB SIPMS Inside The HOUSING WITH CABLES LEADING TO THE READOUT ELECTRONICS SYSTEM

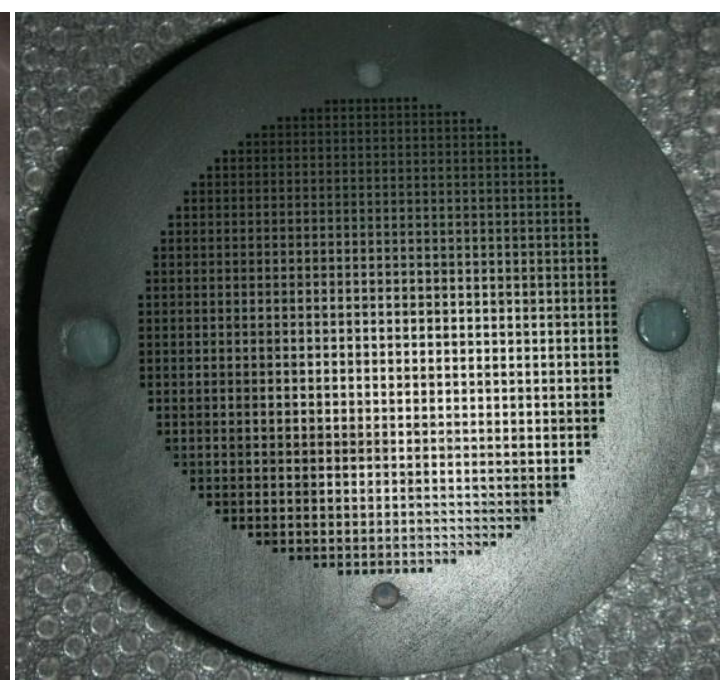

Figure 3:Parallel hole COLLIMATOR

\subsubsection{Image Acquisition and Display Software for Gamma Modality}

The gamma modality is controlled by the data acquisition programming environment KMAX. Figure 4 shows the gamma camera GUI while imaging a patient during a clinical SLNb study. The two imaging windows in the top row show a persistence mode image in the 10x binning mode (left) and a normal acquisition mode image in the 5x binning mode (middle). Operation in persistence mode presents only the regions of highest instantaneous concentration of the radiotracer to the surgeon, enabling an approximate real time imaging capability. Binning is a process where clusters of pixels in an image are combined into a single pixel, resulting in a smaller image of higher intensity values. The concept of image binning is explained in detail in chapter 3. The buttons on the right in Figure 4 allow the user to start/stop the acquisition, scale the display, and clear the imaging windows. In the bottom row, the energy spectra for both images are shown, and the limits on the energies of the accepted events can be adjusted to reject scattered events. Gamma rays being emitted from the tracer undergo reflection in the medium of 
travel (such as tissue and blood) resulting in scatter. The raw image is shown in the "Control Panel" tab, but this feature was deemed less desirable by the surgeons during the clinical study. When compared to the binned images, the raw images lack of clarity caused by the low acquisition statistics in a typical acquisition time of one minute. The matrix size of the raw images is $270 \times 270$ pixels for the FOV of $60 \mathrm{~mm}$, resulting in an effective pixel size of 0.24 $\mathrm{mm}$.

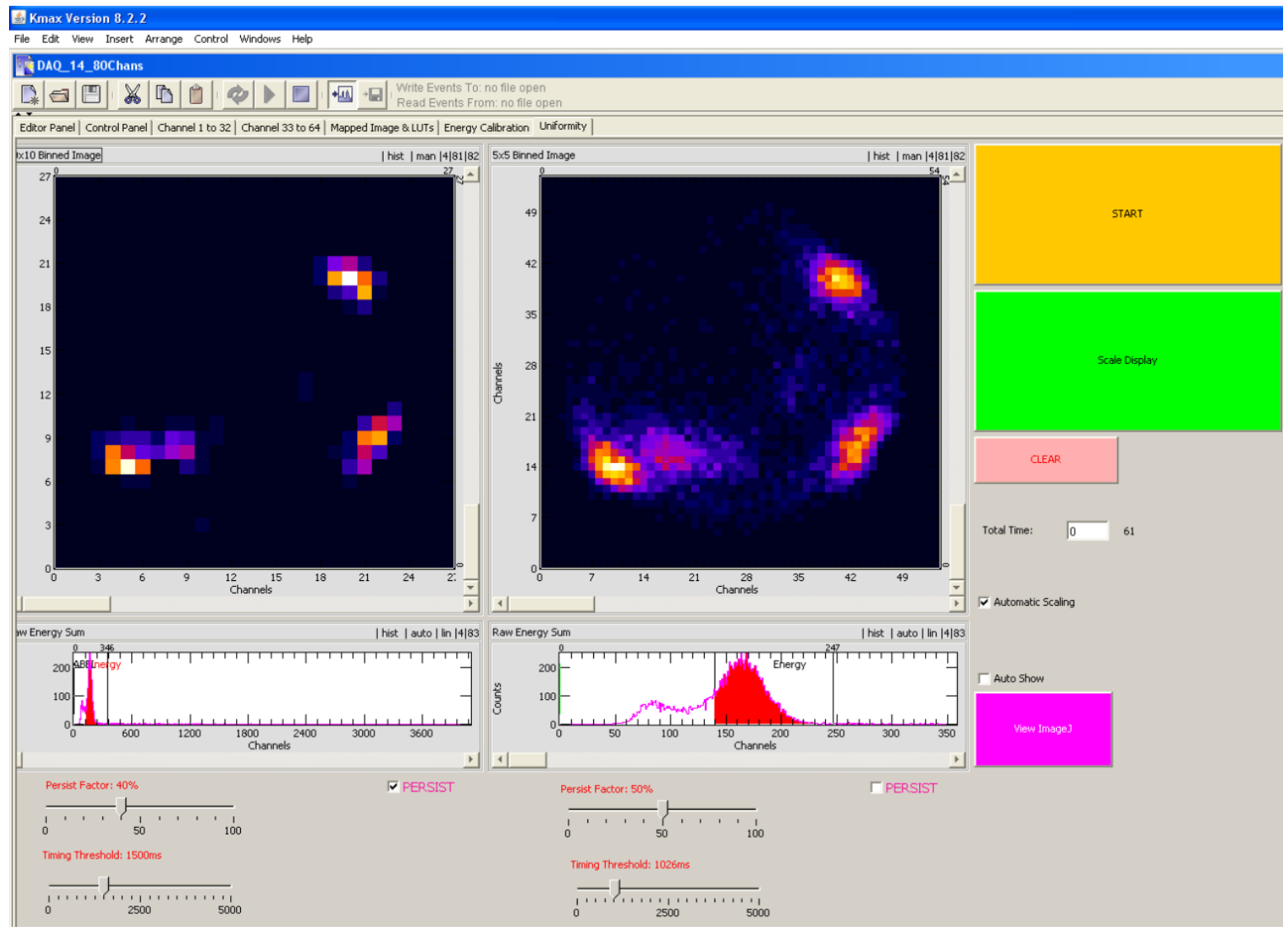

FIgURE 5: SCREENSHOT OF THE GAMMA IMAGE ACQUISITION/DISPLAY SOFTWARE

\subsection{Visible/ Near Infrared Fluorescence Imaging Modality}

Fluorescence is a process of light emission by certain molecules (fluorophores) after they have absorbed light or other form of energy. It is a form of luminescence and is usually a result of a 
three-stage process illustrated by the electronic state diagram in figure 5 below. Initially, the fluorophore is in its ground state $S_{0}$ and is supplied energy from an external source, usually in the form of light. With the energy being absorbed by the fluorophore, the electrons from the ground state are excited into one of the vibrational levels of an excited singlet state. This excited state exists for a short amount of time (on the order of $10 \mathrm{~ns}$ ), during which the electron is subject to losing some energy due to interactions with its environment, among other processes, that can depopulate $S_{1}$. The photon is then emitted spontaneously when the fluorophore returns from the lowest vibrational level of the excited singlet state $S_{1}$ to the ground state $S_{0}$. Due to the energy losses mentioned above, the energy of the photon emitted is lower (and wavelength longer) compared to the photon that was absorbed.

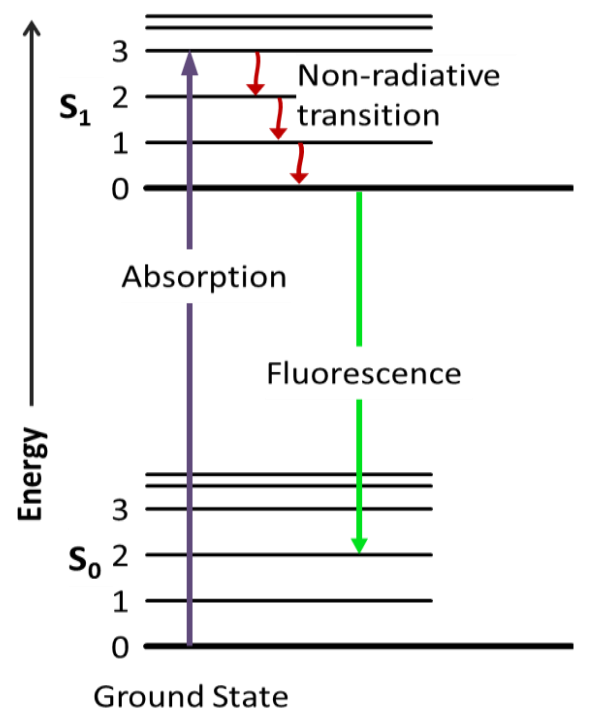

Figure 6: WORKING PRINCIPLE OF FLUORESCENCE

Fluorescence imaging uses a method called planar epi-illumination imaging. It is done by collecting the fluorescence emitted towards the camera by fluorescent objects that were 
illuminated by a plane wave. In this method, the illumination light of appropriate wavelength reaches the surface of the object being imaged and can propagate for several millimeters under the surface to excite shallow and sub-surface fluorophores. The fluorescence light is emitted in an isotropic fashion, and a part of it can be captured by an appropriate detector. Some of the important characteristics of fluorophores are the excitation (absorption) and emission spectra, quantum yield, and quenching properties and limits. The spectrum of the illumination/excitation light should be as close to the peak and shape of the absorption spectrum of the fluorophore to allow for optimal excitation. For this purpose, indocyanine green (ICG) is used as the fluorophore.

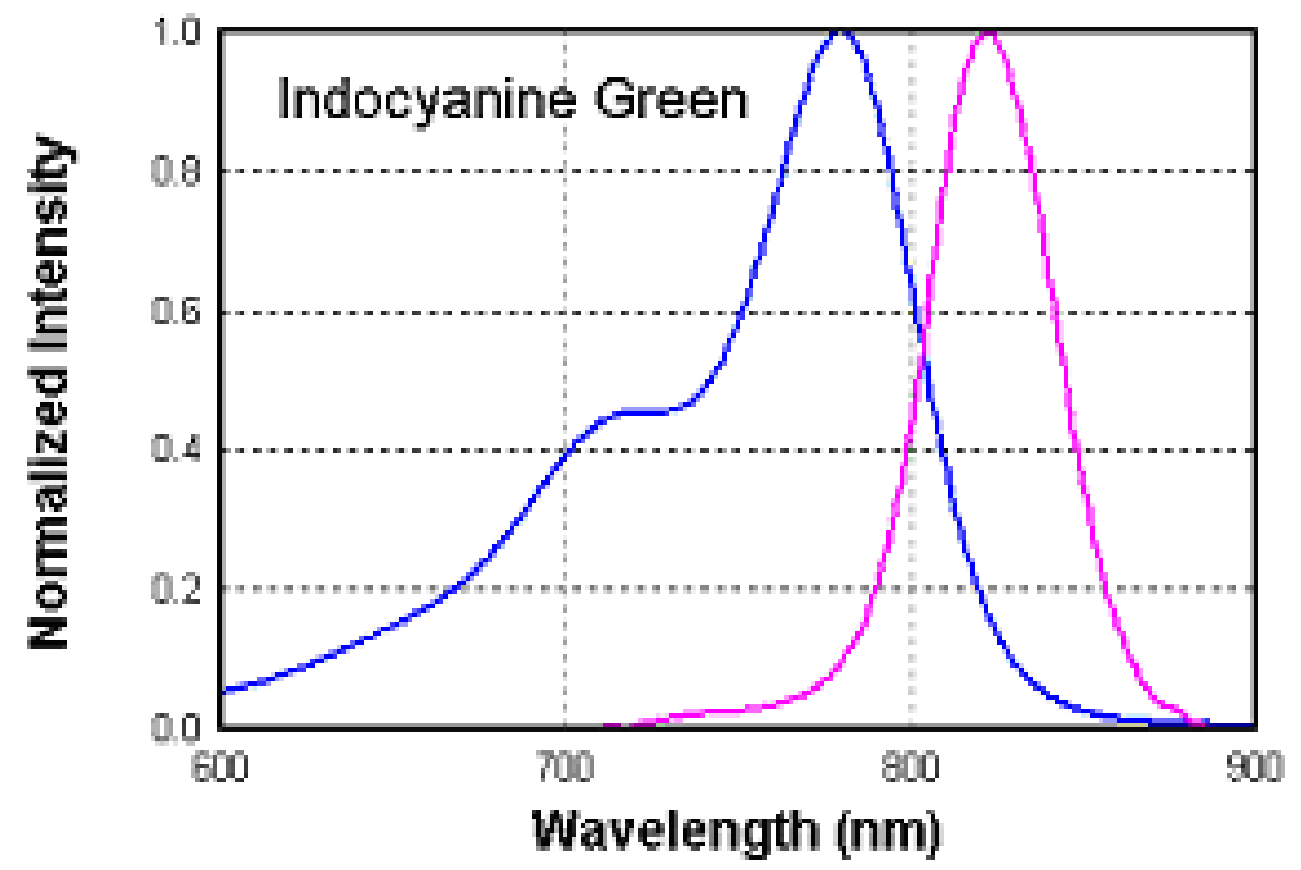

Figure 7: Absorption (blue) ANd EMission (PURPLE) SPECTRA OF INDOCYANine green (ICG) IN WATER 


\subsubsection{Visible/ Near Infrared Fluorescence Imaging System Assembly}

The visible/NIRf imaging system consists of a camera which can image within both visible and NIR spectral ranges, a set of LEDs in the NIR range to excite the fluorophore, and a readout system. Regular lights in the operation room emit a significant amount of radiation in the NIR, which can give false data. Therefore, the system also has a set of LED lights that emit visible light to be used instead of the operation room lights.

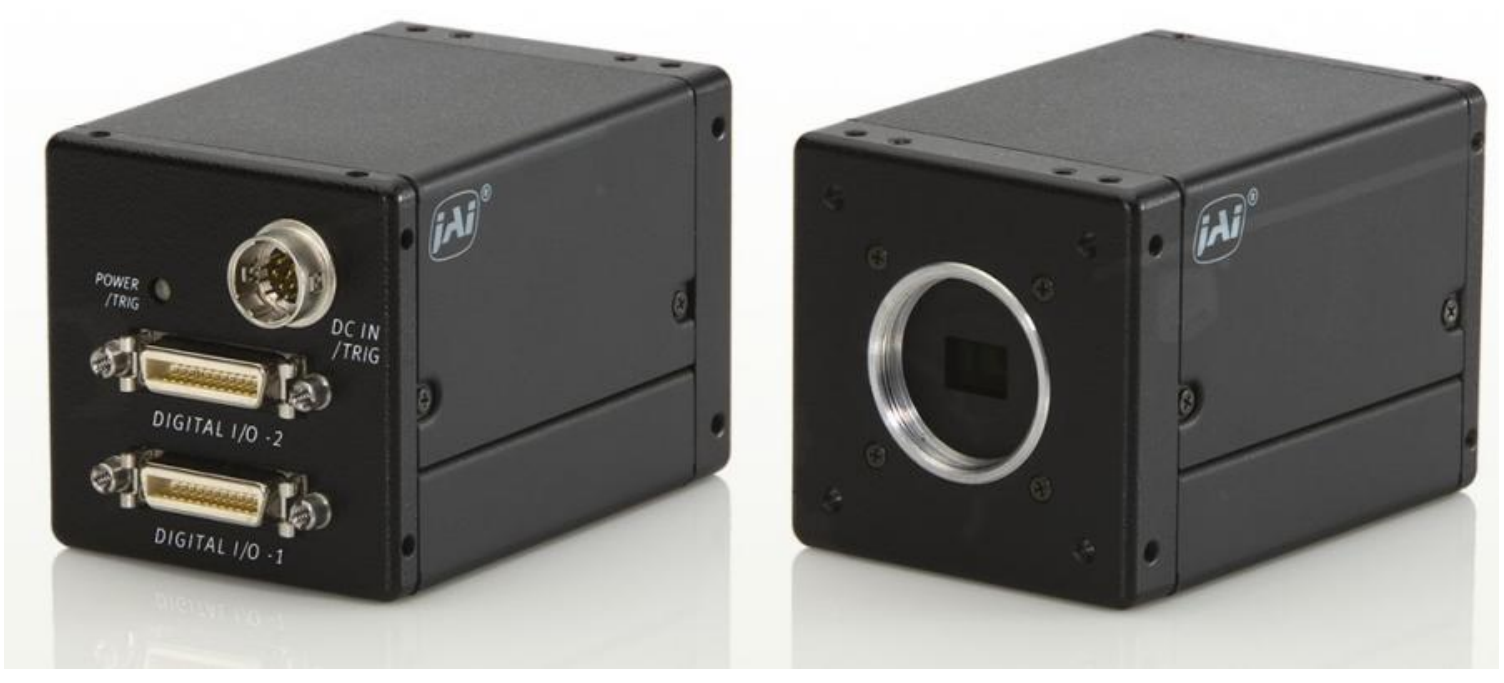

Figure 8: Visible/NIRF CAMERA

A charge coupled device (CCD) is a silicon-based circuit, consisting of densely packed photodiodes that convert light energy into electronic charge. This camera uses two CCDs to image the visible and NIR light. A shared optical path and a 1/3" dichroic prism allow for precise separation of the visible (400-700 nm) and near infrared (700-1000 nm) spectra. In order to focus the image properly onto the CCD and collect the fluorescent light emitted from the imaging field efficiently, a lens is placed in front of the camera. The camera has an image matrix size of 1024 (h) $x 768(w)$ with a pixel size of $4.65 \mu \mathrm{m}$. The temporal resolution of the camera is up to 30 
frames per second (fps), with several options for a shortened exposure time or faster frame rate up to $60 \mathrm{fps}$ in partial scans. The camera is generally operated in a continuous (free-run) mode.
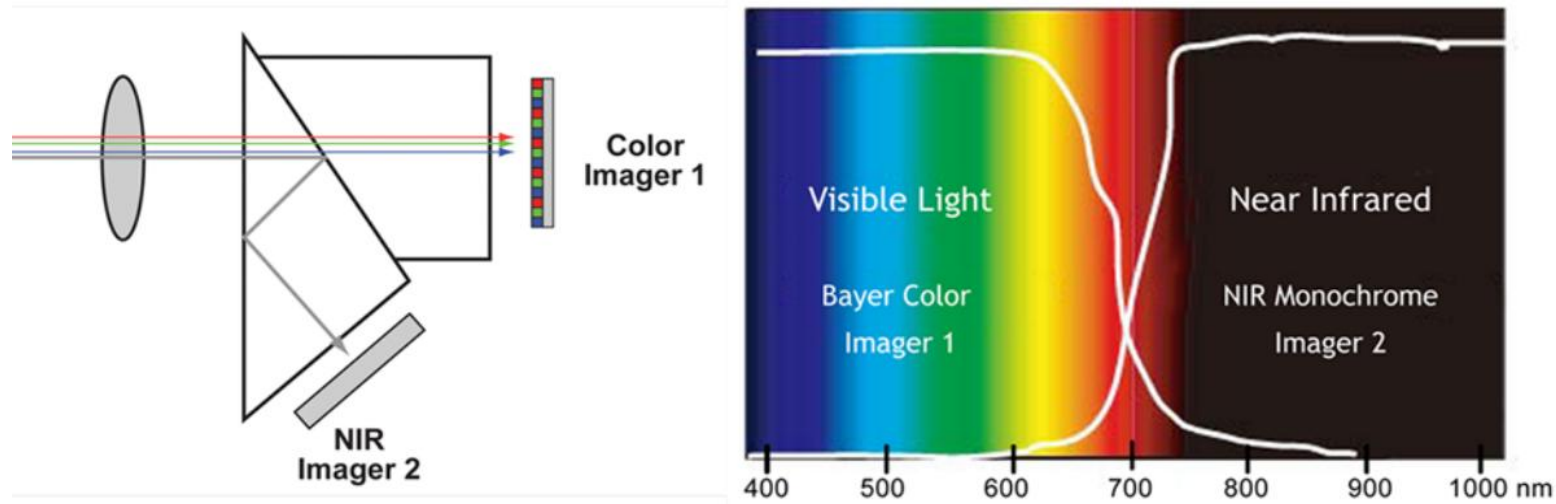

Figure 9: SEPARATION OF VISIBLE AND NIRF IMAGES

The LED spectrum has to match the absorption spectrum of the fluorophore (ICG), which is currently the only NIR fluorophore approved by the U.S. Food and Drug Administration (FDA) for use in human studies. Thus, four high power NIR LED modules with a peak wavelength of the emission spectrum at $760 \mathrm{~nm}$ with the full width at half max (FWHM) of $30 \mathrm{~nm}$ are used. To operate the LED within the safe temperature range required by the manufacturer $\left(30^{\circ} \mathrm{C}\right.$ to 80 ${ }^{\circ} \mathrm{C}$ ), a heat sink is utilized to dissipate the heat away from the LEDs during continuous operation.

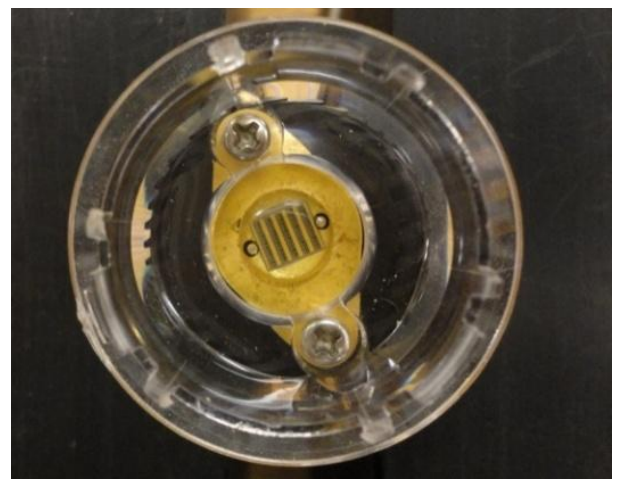

FIGURE 10: NIR LED

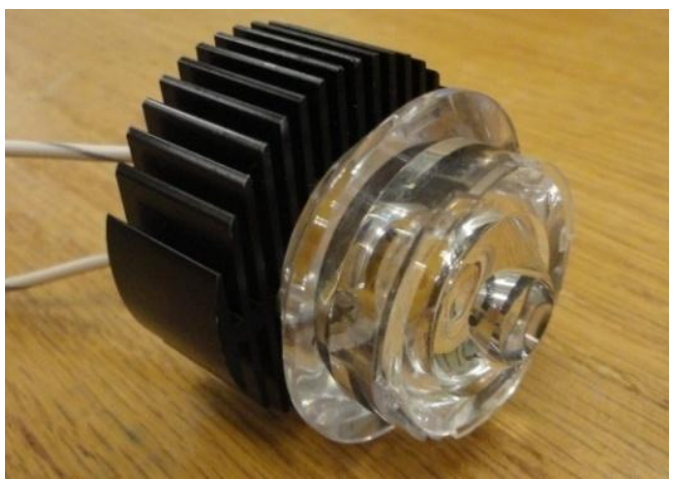

FIGURE 11: NIR LED WITH HEAT SINK 
The room and overhead lights in the operating room have a strong NIR component that saturates the NIR CCD. Hence, three RGB LEDs are used in the IMIS in the place of the room and overhead lights, with each LED providing 418, 599 and 169 lumens, respectively of red, green and blue light and have large heat sinks.

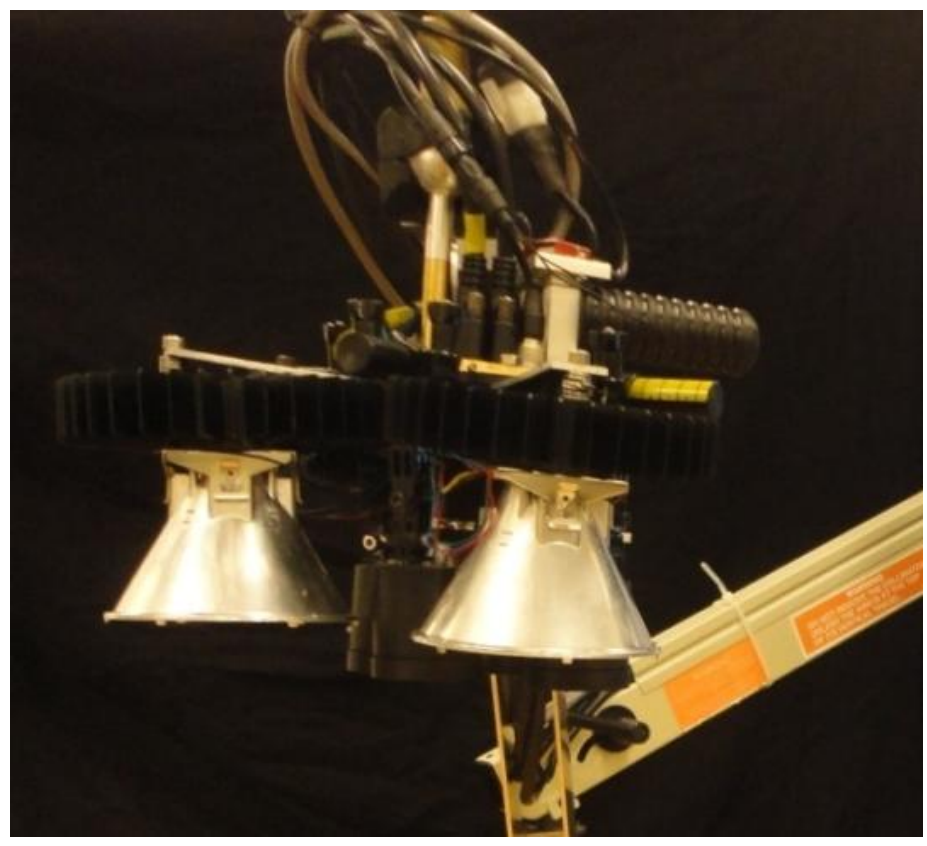

Figure 12: RGB LEDS WITH HEAT SINKS

\subsubsection{Image Acquisition and Display Software for Visible/NIRf Modalities}

The acquisition and the display for the visible/NIRf modality are carried out through LabVIEW. A screen shot of the graphical user interface (GUI) of the visible/NIRf sub-system is shown below in figure 12. The top left window in this figure shows a visible only image (grayscale), and the top right window shows the NIRf only image. The combined gamma, visible and NIRf image is shown in the bottom left window with the fluorescence signal shown in green and the gamma outline (not enabled by the user in this example) colored red. The controls in the bottom 
right area allow the user to perform a range of visual adjustments to the combined visible/NIRf image, including changing the transparency of the NIRf image and adjusting the window and level of the NIRf image that is shown in the fused image. The user can also choose to record the video, display the gamma image ROI if desired, and save a snapshot of the images.

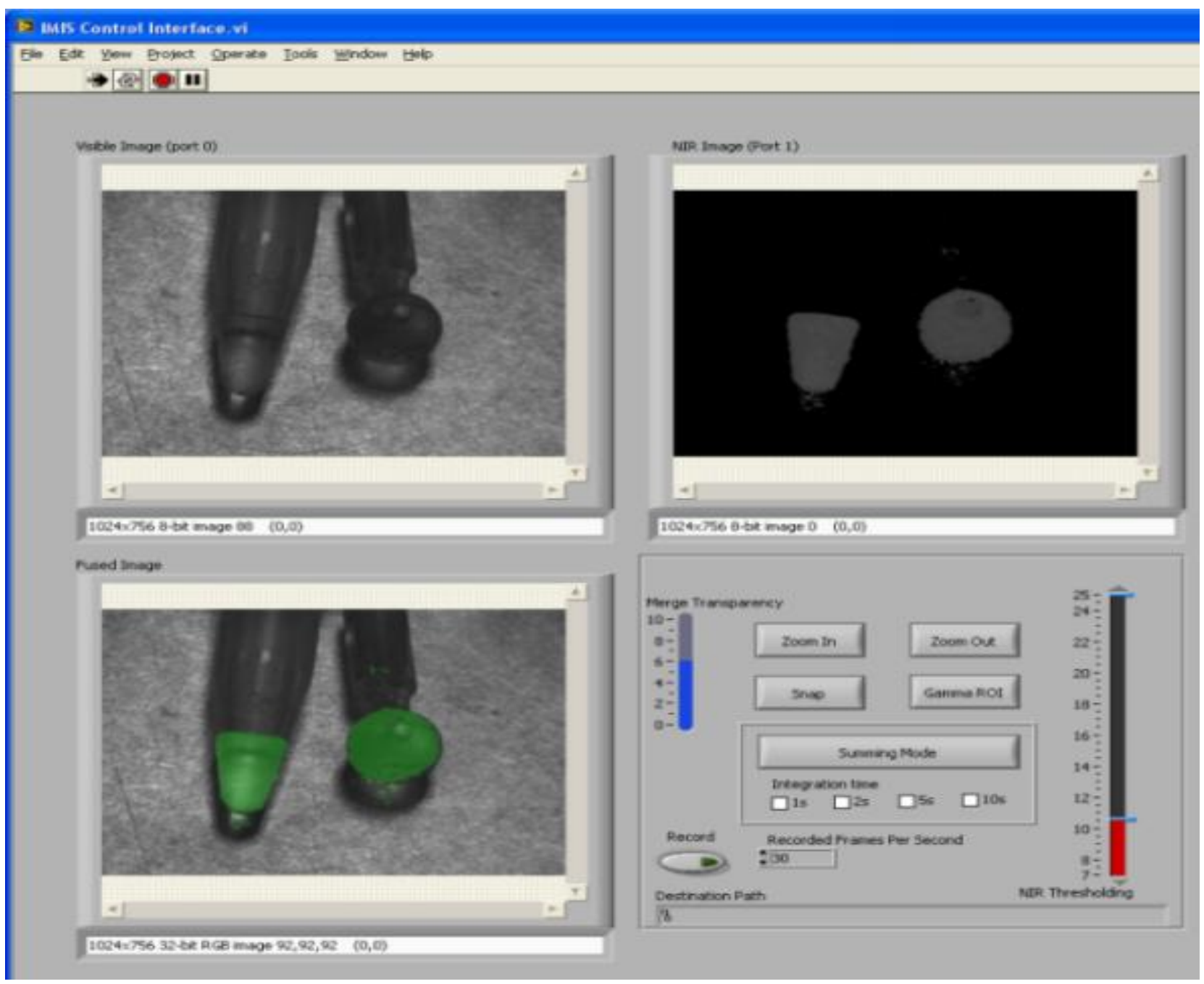

Figure 13: SCREENSHOT OF LABVIEW BASED GUI FOR THE ACQUISITION AND DiSPLAY OF THE DATA FROM THE VISIBLE/NIRF MODALITY 


\section{CHAPTER 3: GAMMA IMAGE SEGMENTATION}

IMIS aims to combine the gamma and visible/NIRf images to provide more complete information about the location and extent of the targets of interest. Gamma images are acquired by imaging a tracer $\left({ }^{99 \mathrm{~m}} \mathrm{Tc}\right.$-pertechnetate $)$ injected into the patient. They are saved through a program called KMAX. A gamma image is a collection of discrete points which gives only functional information (the amount of radioactivity) in the target. It would obscure the visible image if overlaid directly. Hence, the image is segmented and the outline of the region of interest (ROI) is overlaid on the visible/NIRf image. The entire gamma image segmentation process is done in MATLAB.

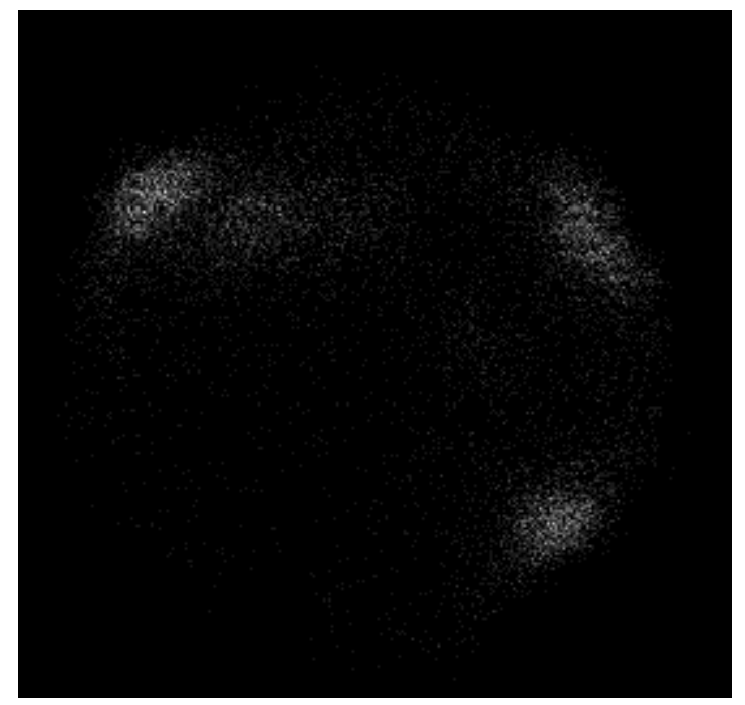

Figure 14: EXAMPle of a gamma image

\subsection{Pre-processing}

The data in a gamma image is saved in a text file as an array of numbers where each number represents the scaled intensity value detected by a given pixel. This intensity is proportional to 
the number disintegrations of the radioisotope. KMAX, by default, stores images as a $1400 \mathrm{x}$ 1400 array whereas the actual image is much smaller $(270 \times 270)$. This padding results in a significant amount of unnecessary blank data points. The file also has a header which is not useful for this application. Hence, the first step is to read the file, remove the unnecessary data, and convert it to a grayscale image.

Gamma images inherently have background noise, and the data points are usually sparse and discrete. These factors could potentially affect the segmentation process. In order to reduce the noise, a standard Gaussian filter is used. A Gaussian function with real constants a, b, and c is of the form

$$
f(x)=a e^{-\frac{(x-b)^{2}}{2 c^{2}}}
$$

which is a symmetric "bell curve" shape. Mathematically, a Gaussian filter modifies the input signal by convolution with a Gaussian function to smooth any small regions of high intensity gradient, reducing the possibility of detecting false edges.

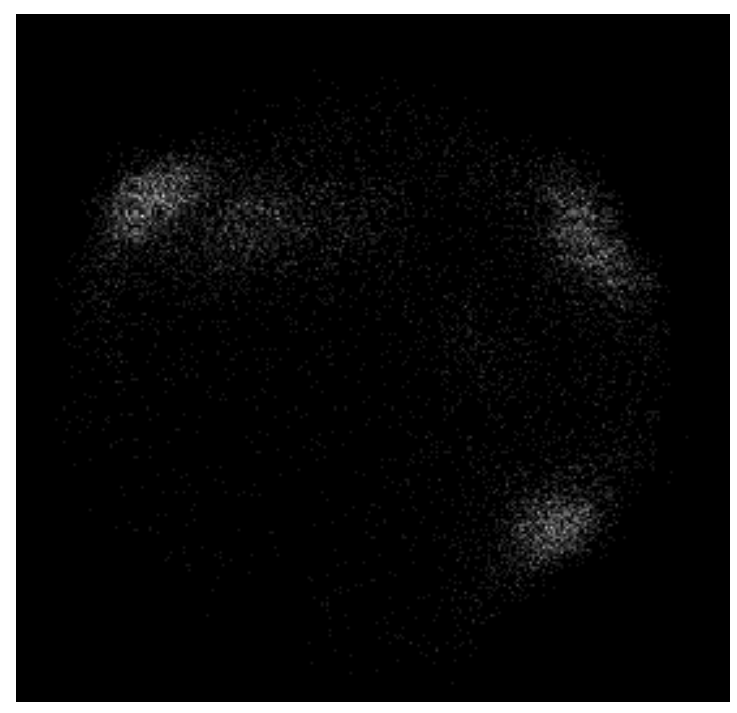

FIgURE 16: RAW IMAGE

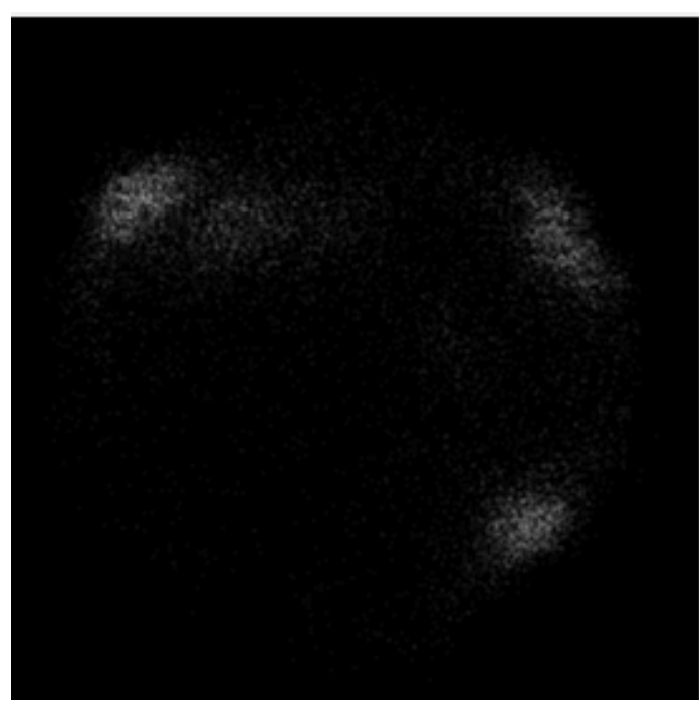

Figure 15: Filtered image 


\subsection{Image Binning}

To reduce the effects of minor observation errors, a method called data binning is used. For each data point location which is the central location, all the values in its neighborhood are considered to be in a bin. The value at the central location is replaced by a resultant of all the values in the bin. It is a form of quantization. In image processing, binning is the procedure of combining a cluster of pixels into a single pixel. In $\mathrm{nXn}$ binning, an array of $\mathrm{n}^{2}$ pixels becomes a single larger pixel, reducing the overall number of pixels. This aggregation, reducing the number of data, facilitates the analysis. It gives a lower resolution image with reduced noise. (13).

For the current application, binning is used to club the sparse data together to form denser images. The value of the resultant pixel intensity is calculated as the sum of the individual intensities of all the pixels in the bin. The resultant image is smaller, denser, and easier to segment. The effect of binning on an image is shown in figure 16. The leftmost image is the original raw image. Going from left to right, the images are $2 \mathrm{x}, 3 \mathrm{x}$, and $4 \mathrm{x}$ binned, respectively. $2 \mathrm{x}$ binning works best for most images but the program has been written such that it can be easily changed. The lower the imager statistics, the higher the binning that is to be used.

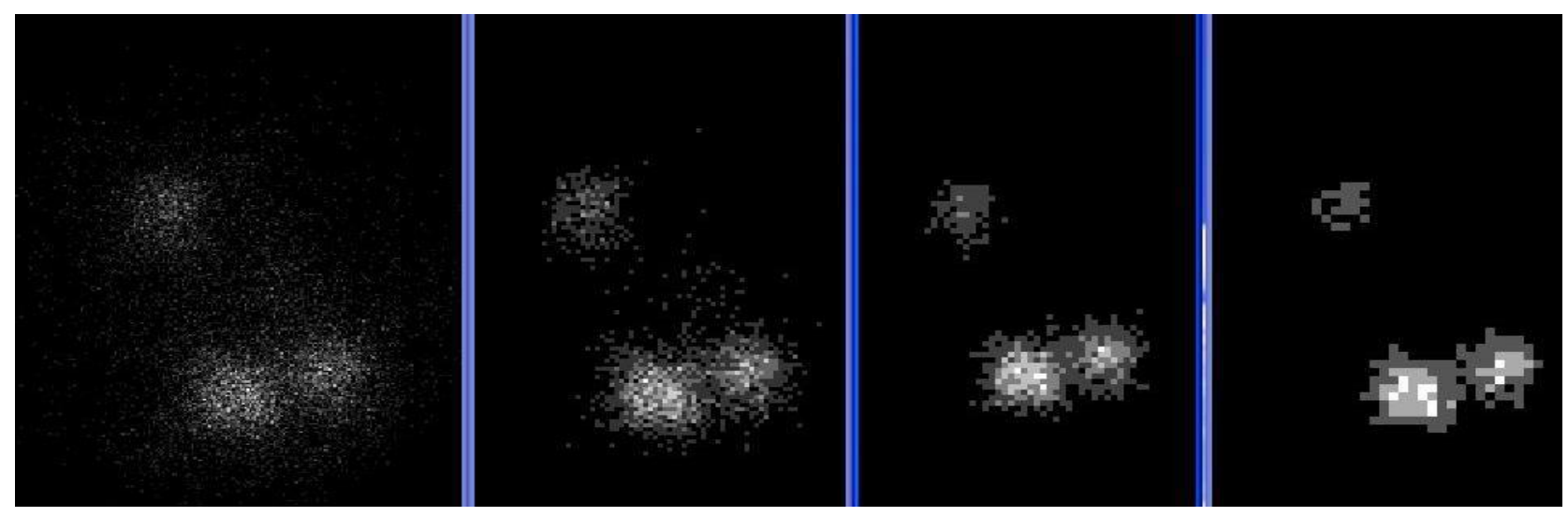

Figure 17: EXAMPLES OF BINNING RAW IMAGES (FROM LEFT TO RIGHT 1X, 2X, 3X AND 4X) 


\subsection{Image Binarization}

Various edge detection algorithms work best on binary images. As such, the gamma images are converted to binary format. A binary image is a digital image that has only two possible values for each pixel. Binary images are also called bi-level or two-level, meaning each pixel is stored as a single bit (0 or 1) (14). To convert a grayscale image to a binary image, thresholding is used. All pixels with intensities above the threshold are converted to a binary 1 (white) and those below are converted to binary 0 (black). From our previous clinical study data, $30 \%$ of the maximum intensity in a particular image was calculated as the ideal threshold value.

The binary image would have small regions of 0 s or $1 \mathrm{~s}$ as a result of noise or other image imperfections. The next step is to fill these small holes (clusters of 0s) and remove small islands (clusters of 1s) to get a clean binary image. This step is performed by selecting a limiting value for the minimum cluster size. Any cluster of pixels of value 0 whose size is less than the limiting value would be converted to 1 and vice-versa. An example of image binarization is shown in figure 17.
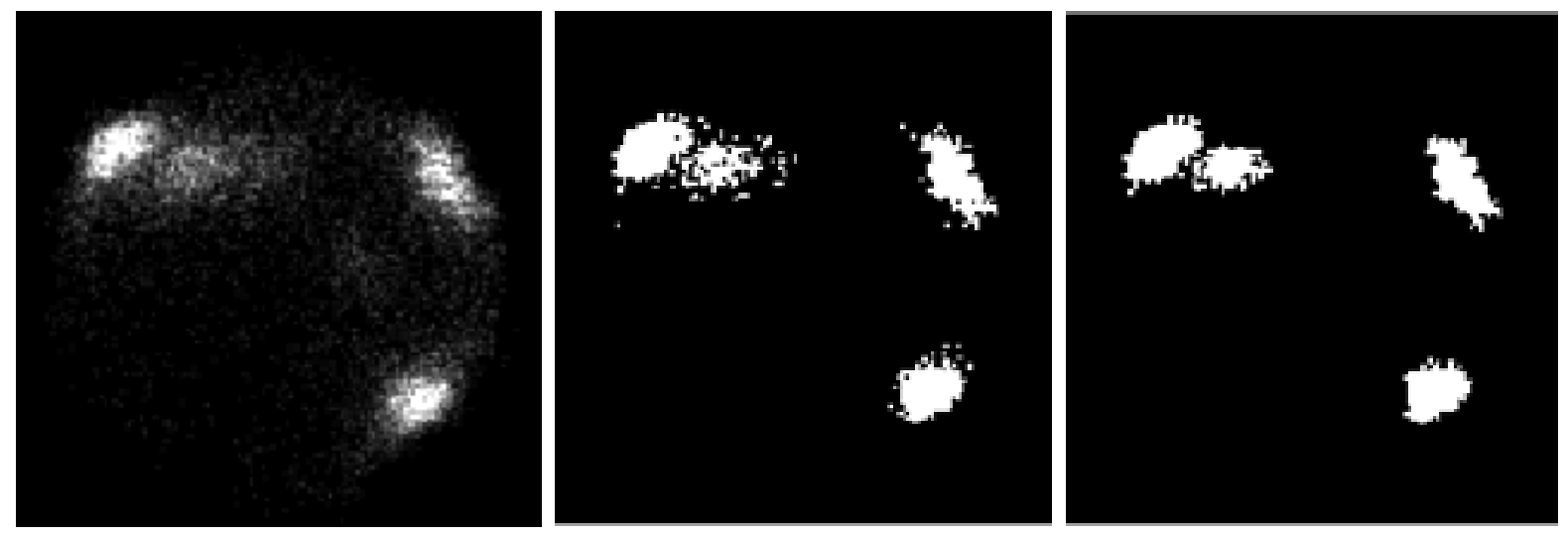

Figure 18: Binned IMAge, Binary IMAge, BinARy IMAge- Cleaned (LeFt To RIGHT) 


\subsection{Canny Edge Detection Algorithm}

The Canny edge detector is an edge detection operator that uses a multi-stage algorithm to detect a wide range of edges in images. Because the Canny edge detector is susceptible to noise present in raw unprocessed image data, it uses a filter based on a Gaussian. The result is a slightly blurred version of the original image, which is not affected by a single noisy pixel to any significant degree. An edge in an image may point in a variety of directions, so the Canny algorithm uses four filters to detect horizontal, vertical and diagonal edges in the blurred image. The edge detection operator returns a value for the first derivative in the horizontal direction $\left(G_{x}\right)$ and the vertical direction $\left(\mathrm{G}_{\mathrm{y}}\right)$, from which the edge gradient and direction can be determined:

$$
\begin{gathered}
\mathbf{G}=\sqrt{\mathbf{G}_{x}{ }^{2}+\mathbf{G}_{y}{ }^{2}} \\
\Theta=\arctan \left(\frac{\mathbf{G}_{y}}{\mathbf{G}_{x}}\right) .
\end{gathered}
$$

The edge direction angle is rounded to one of four angles representing vertical, horizontal and two diagonal directions ( $0,45,90$ and 135 degrees for example). Given estimates of the image gradients, a search is then carried out to determine if the gradient magnitude assumes a local maximum in the gradient direction. From this stage, a set of edge points, in the form of a binary image, is obtained. These are sometimes referred to as "thin edges".

Large intensity gradients are more likely to correspond to edges than small intensity gradients. It is in most cases impossible to specify a threshold at which a given intensity gradient switches from corresponding to an edge into another feature. Therefore, Canny uses thresholding with hysteresis. Thresholding with hysteresis requires two thresholds - high and low. Making the assumption that important edges should be along continuous curves in the image allows us to 
follow a faint section of a given line and to discard a few noisy pixels that do not constitute a line but have produced large gradients. Therefore, high threshold is first applied. This process marks out the edges that are more likely to be genuine. Starting from these locations, using the directional information derived earlier, edges can be traced through the image. While tracing an edge, the lower threshold is applied, allowing faint sections of the edges to be traced as long as it has a starting point. Once this process is complete, a binary image is obtained where each pixel is marked as either an edge pixel or a non-edge pixel (15). The main advantage of the Canny edge detector is that it gives fairly continuous edges. The use of hysteresis thresholding ensures that even edges of smaller intensity gradients are detected while the noise is suppressed. A high threshold of 0.7 and a low threshold of 0.28 have been used in this algorithm. An example of the output of the Canny edge detection algorithm is shown figure 19.

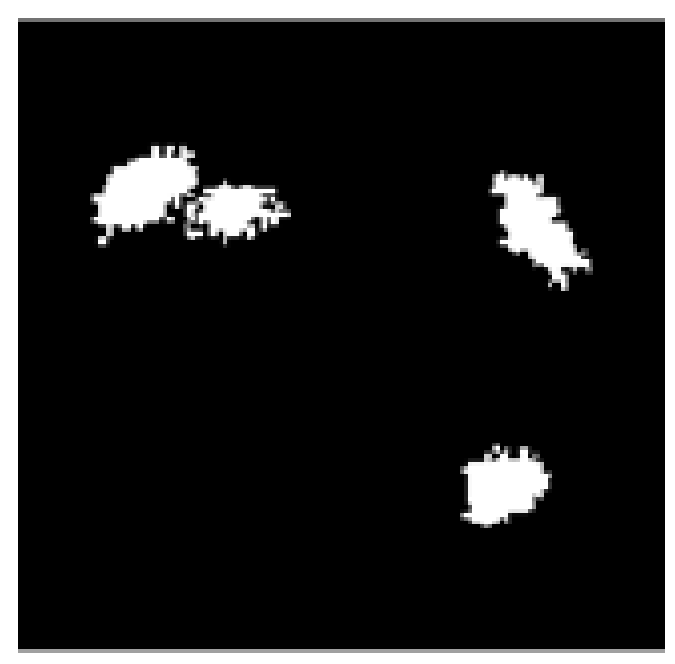

FIgURE 20: BINARY IMAGE

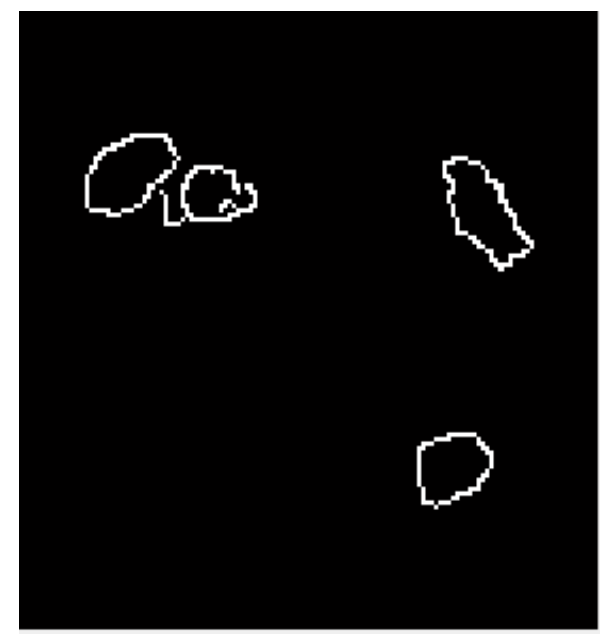

Figure 19: CANny edges deteCted FROM THE BINARY IMAGE 


\subsection{Active Contour Segmentation- Snakes}

A snake is an energy-minimizing spline guided by external constraint forces and influenced by image forces that pull it toward features such as lines and edges. Snakes are active contour models: they lock onto nearby edges, localizing them accurately (16). Snakes have been widely used for image segmentation. The active models deform on the image domain and capture a desired feature by minimizing an energy functional subject to certain constraints. The energy functional usually contains two terms: an internal energy, which constrains the smoothness and tautness of the model, and an external energy, which attracts the elastic model to the features of interest (FOI) (17).

In this application, vector field convolution (VFC) based external energy is used. This external force is calculated by convolving a vector field with the edge map derived from the image. The external energy algorithm was developed by Dr Scott Acton and Bing Li at the University of Virginia. Snakes are used for semi-automatic segmentation. A seed point is initialized and the contour grows until it reaches a point of minimum energy, which marks the boundary of the region. In the current application, snakes are used on the edge map obtained from Canny edge detection. The purpose of using snakes for segmentation is two-fold:

1) In images that have multiple ROIs, the surgeon may want to see only one or more specific regions on the final fused image. Snakes gives the surgeon the capability of selecting which ROIs they want to see on the final visible image.

2) The edge map obtained from Canny edge detection might be discontinuous and may contain some edges detected because of noise. Snakes always give closed contours and are not affected by noise, so they are used to obtain smooth and closed edges. 
An example of the application of snakes is shown in figure 20. In this example, the gamma image has four hot spots. Canny edge detection has been applied to get boundaries of the four regions shown in figure 21 . The user decides to have only one of these boundaries on the final image by initializing a snake in the centre of the required boundary. The snake deforms to segment only that boundary region. If the user wants to segment all the ROIs detected by Canny, it can be done in two ways.

1) By clicking on the center of each ROI such that a snake is initialized at each location

2) By choosing the automated option. The center of gravity of each ROI is calculated and passed as an initialization point to a snake. This option can pose difficulties in images which have significant noise, resulting in too many seed points.

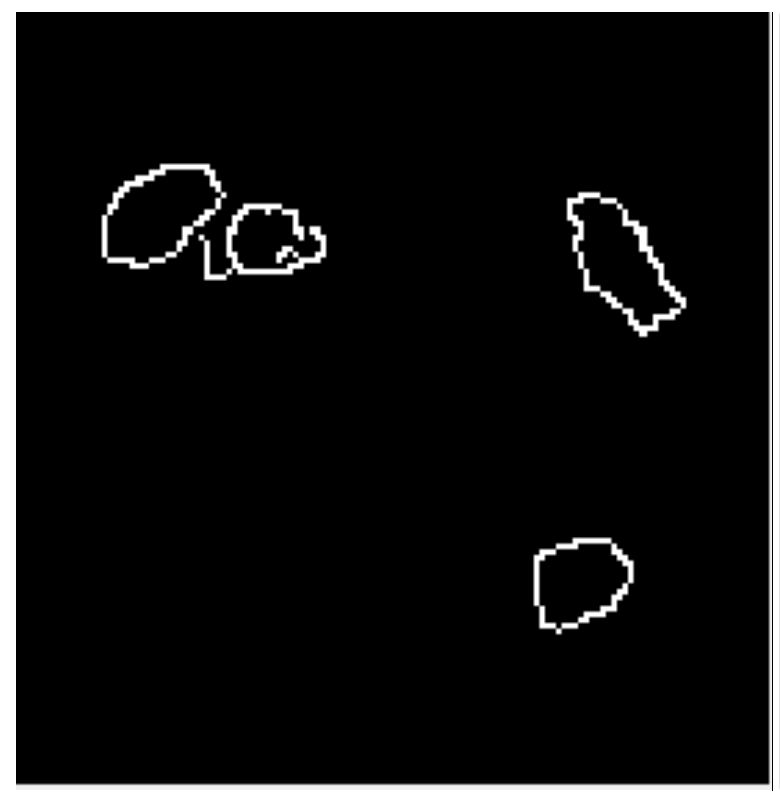

FigURE 22: EDGE MAP FROM CANNY EDGE DETECTOR

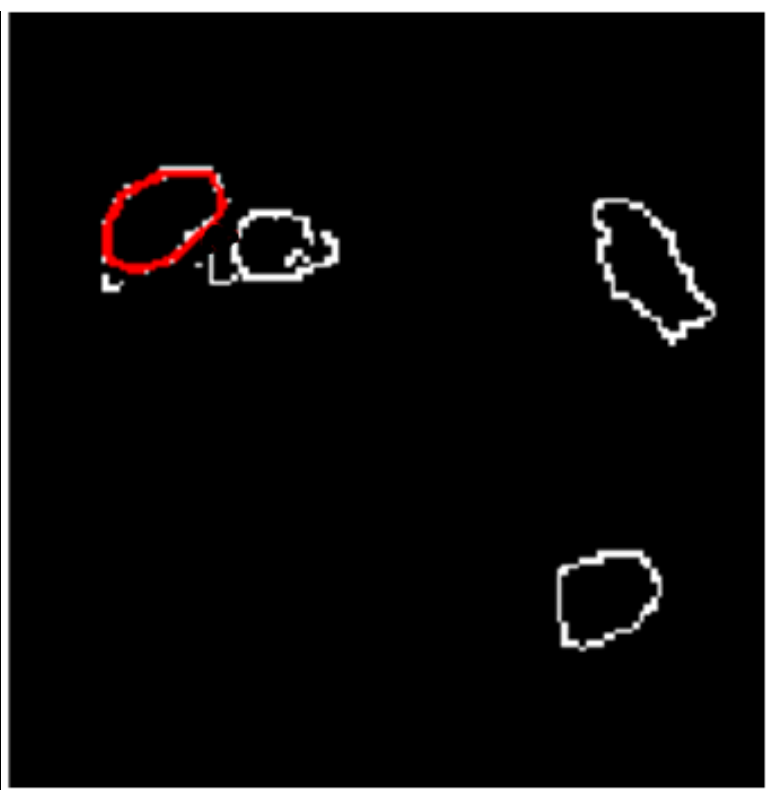

Figure 21: Final ROI SEgmented BY THE SNAKE IS SHOWN IN RED 


\subsection{Calculating the Number of Gamma Ray Counts}

In addition to providing a boundary of ROIs to aid surgical incisions, the segmentation algorithm can also calculate of the number of gamma ray counts. The standard for lymph node mapping is that the surgeon removes all lymph nodes from the patient whose count rate in-vivo exceeds one tenth of the count rate of the hottest lymph node ex-vivo. For this purpose, count rate measurements are usually done with a non-imaging surgical probe. Due to the properties of this probe, it leads to inherent errors in the process and likely operator bias. These issues can be resolved by using the gamma camera and the segmentation algorithm.

After the desired ROIs are selected and segmented using snakes, a new image is created, which contains only the final ROIs. This image is converted into a mask and overlaid on the original gamma image. The number of counts within that boundary is calculated by adding all the individual pixel intensity values. This sum can be used to estimate the activity in the selected region. Radioactivity is measured in units of Becquerels $(\mathrm{Bq})$, defined as the number of disintegrations per second (counts per second). The activity in Bq within the ROI can be calculated by dividing the number of counts by the acquisition time and sensitivity of the gamma camera.

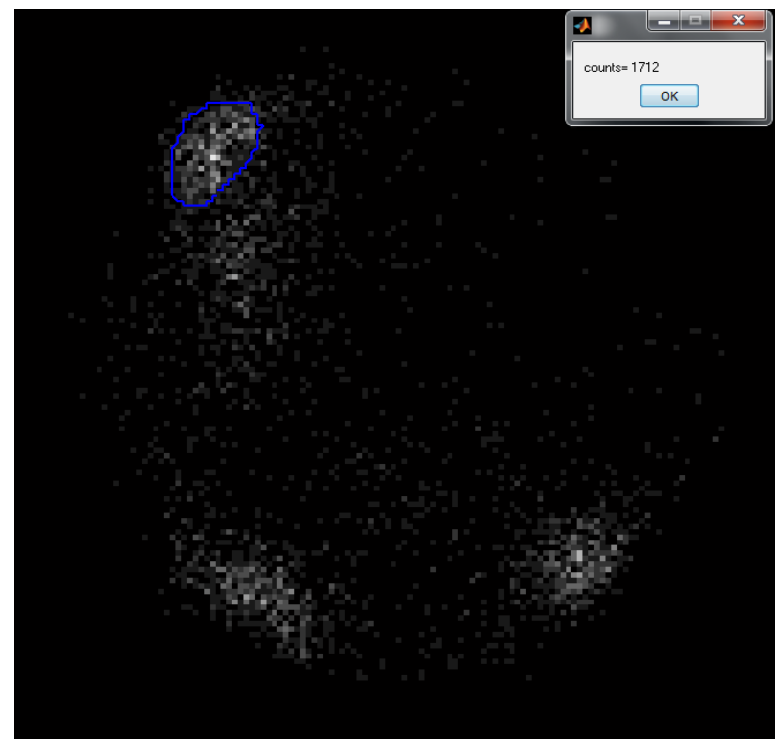

Figure 23: IMAGe of A SEGMENTED NODE WITH THE CORRESPONDING NUMBER OF COUNTS 


\section{CHAPTER 4: IMAGE CO-REGISTRATION}

Image co-registration in IMIS has two steps: co-registration of visible and NIRf images and coregistration of gamma and visible/NIRf fused image. Co-registration of visible and NIRf images is an important, but straightforward, step because the same camera is acquiring both images. Coregistration of the gamma image with the visible/NIRf image is a non-trivial task because both cameras have different resolutions and fields of view (FOVs). Moreover, the fact that the two cameras are free hand in use poses significant difficulty.

The goal of co-registration is to enable the information from the most recently acquired gamma image to be available during the visible/NIRf image acquisition to provide guidance to surgeons until the surgical targets are near the surface. The idea is to show the boundary outlines from radioactive targets in the gamma image on top of the visible/NIRf video, giving the surgeon an idea about the location and extent of targets prior to the incision. Screenshots of the MATLAB GUI developed for performing segmentation and offset correction are shown in figure 23 .

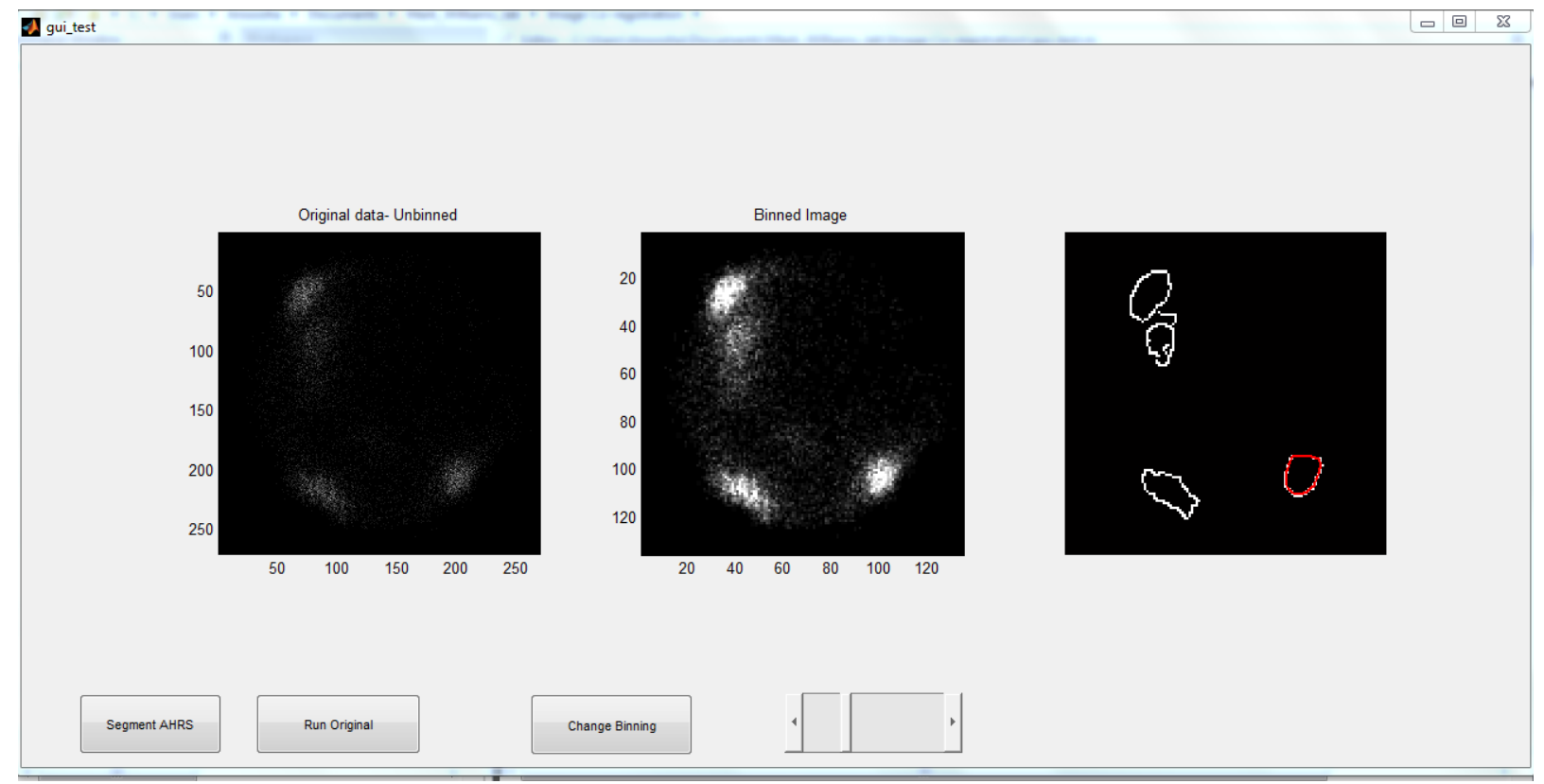


This GUI can be used for both gamma image segmentation and offset correction using the VectorNav logo. In the above figure, if "Run Original" is selected, the program calls the last saved gamma image and performs image segmentation as described in the previous chapters. If the data is too sparse, or the binning has to be changed for any reason, it can be done by selecting "Change Binning". A window pops up and the desired binning value can be entered.

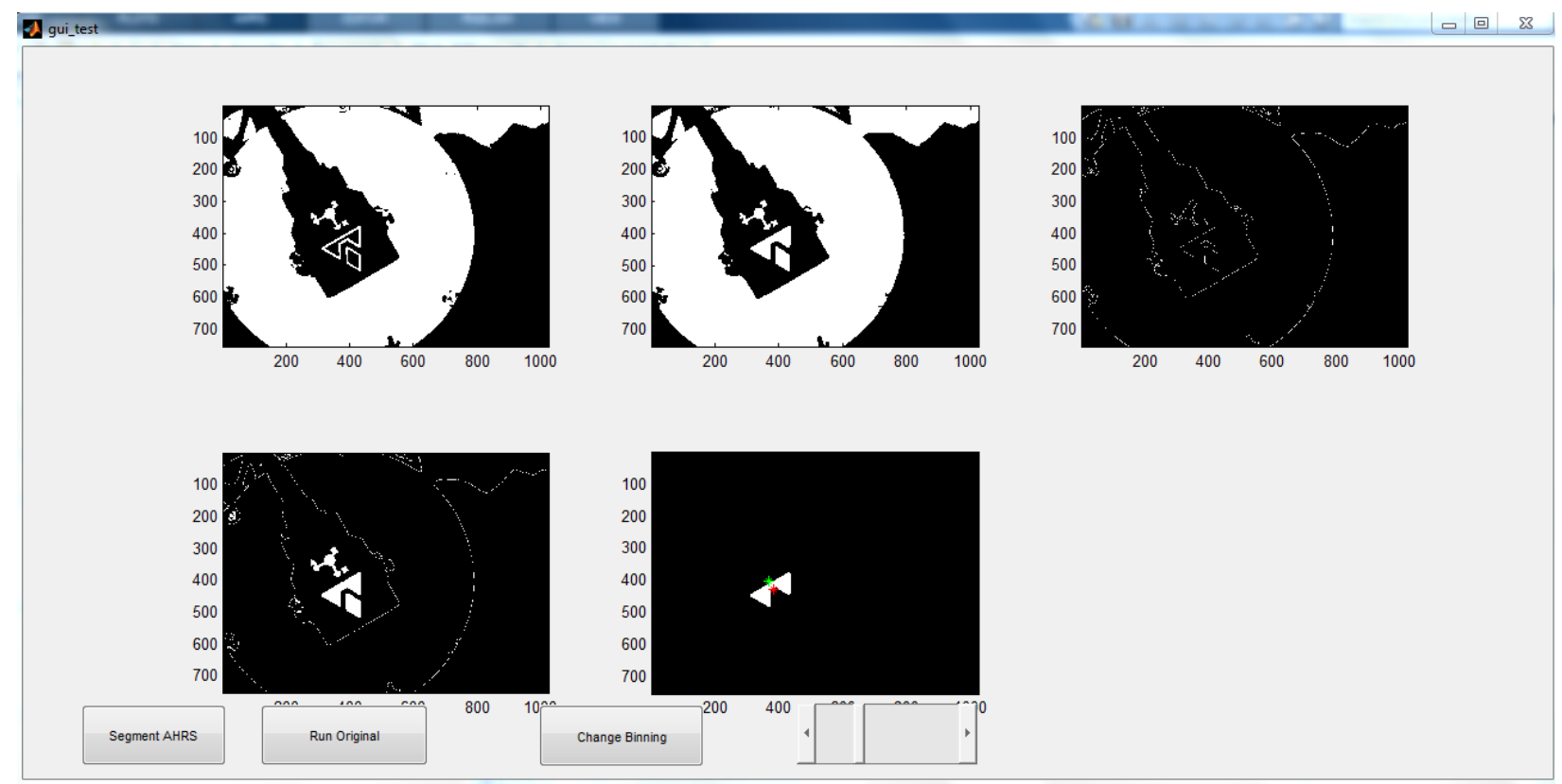

Figure 25: SCREENSHOt OF THE MATLAB GUI DURING OFFSET CORRECTION

To perform offset correction, the logo on the back of the AHRS unit has to be segmented by selecting the "Segment AHRS" button. Care has to be taken to ensure that only the required shape, shown in the bottom right window in figure 24 , is segmented. If more or less area is segmented (shown in the bottom left window), it can be changed by altering the threshold value by moving the slider. 


\subsection{Alignment of the Two Cameras}

A minor misalignment of the gamma camera can cause large errors in the co-registration. These errors are due to the fact that the gamma camera can image targets at considerable depths in the patient, while the visible/NIRf camera, at most, only looks at subcutaneous information as illustrated in the figure 25 . The gamma camera is directly below the visible/NIRf camera, but slightly misaligned in the $\mathrm{z}$ direction compared to it. Due to this misalignment, the two cameras are not looking at the same FOV, and co-registration errors can occur.

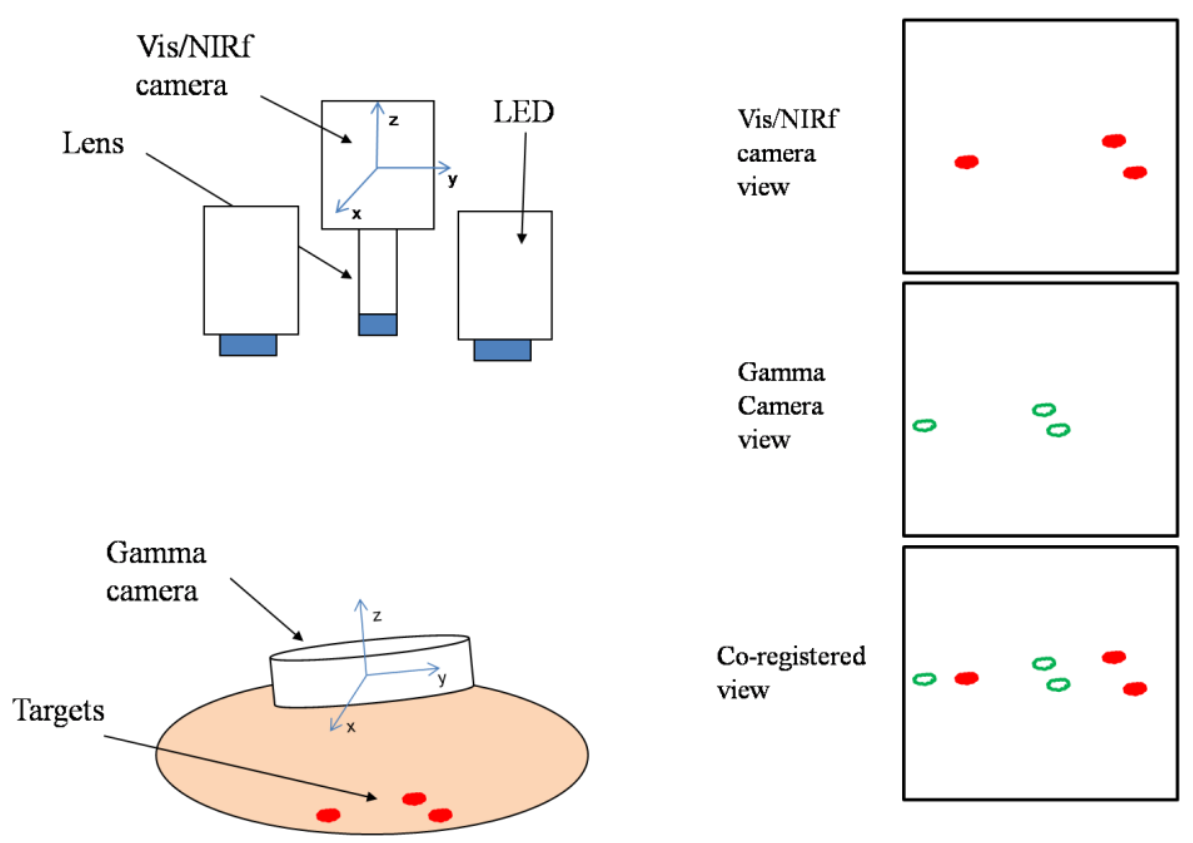

Figure 26:ILlUSTRATION OF THE MISALIGNMENT EFFECT ON THE CO-REGISTRATION ERROR

Alignment of the cameras is achieved with the help of two Attitude and Heading Reference System (AHRS) units. The AHRS units consist of a set of sensors that provide the pitch, roll and yaw (Euler angles) information. The units are mounted with custom adapters on the back of the gamma camera and on top of the visible/NIRf camera head as seen in figure 26. 

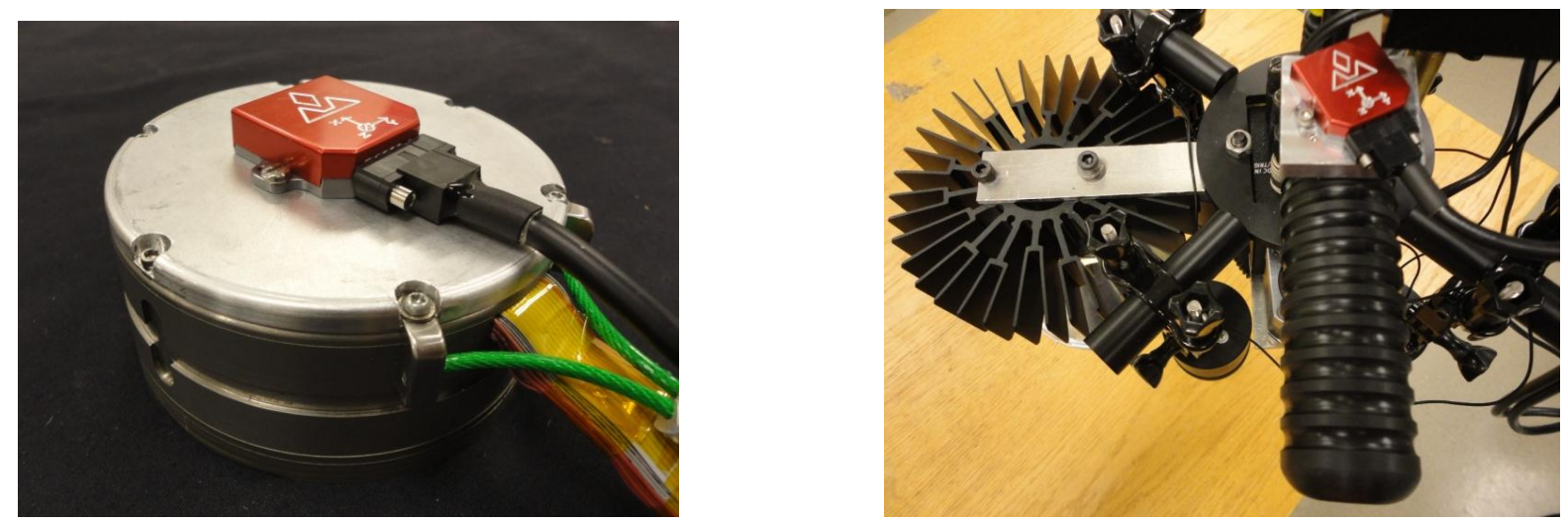

Figure 27: AHRS Units MOUNTED ON THE GAMMA CAMERA(LEFT) AND ViSIBLE/NIRF CAMERA(RIGHT)

The GUI for the AHRS units is a software package provided by the manufacturer VectorNav called Sensor Explorer. A screenshot of the GUI is shown in figure 27. The program shows the coordinate systems of both AHRS units on the screen at the same time and notifies the user when the Euler angles are aligned to within a user defined tolerance value. When the cameras are aligned rotationally, the red "Out of Tolerance" square seen in the figure changes to a green "In Tolerance" display. This software is used to align the two cameras while the gamma camera is acquiring the image.

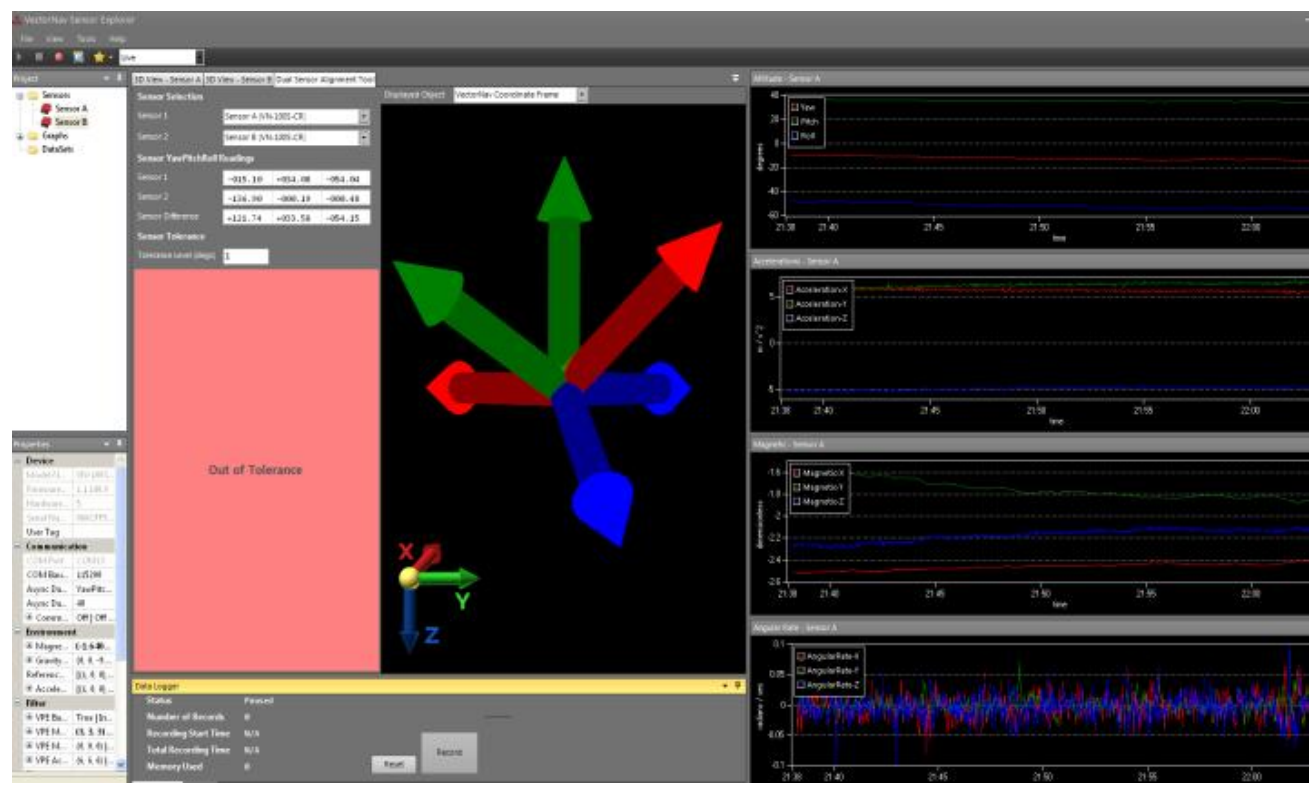

Figure 28: GUI ShOWING THE ORIENTATION VECTORS OF THE AHRS UNitS 


\subsection{Offset Correction}

All of the sub-functions of the image co-registration process are done in MATLAB. Four major differences exist between the gamma and visible/NIRf images: 1) Linear offset between the centers, 2) Resolution (scaling), 3) Rotational offset and 4) Image size. All four are corrected by imaging the back of the gamma camera along with the AHRS unit with the visible camera.

The FOV of the visible camera is bigger than the FOV of the gamma camera. The cameras are positioned such that the entire gamma camera is in the FOV of the visible camera. This placement ensures that both cameras are imaging the same region. Gamma image acquisition takes about $30-60$ seconds. While the gamma camera is acquiring the image, the visible camera is aligned with it and the back of the gamma camera is imaged.

Due to the positioning of the AHRS unit on the visible camera system, there is a rotational offset between the two images, even when the cameras are aligned according to the software. This is a constant angular value as long as the cameras are aligned with respect to the Euler angles given by the AHRS units. The final gamma image containing the outlines of the ROIs is first rotated by this constant angle.

The VectorNav logo on the AHRS unit, which is mounted on the gamma camera, is segmented. The centroid of this logo gives the center of the gamma camera with respect to the visible image. The center of the gamma image is always the center of the gamma camera. Hence, these two locations can be used to find the relative offset between the centers of the gamma and visible images. 


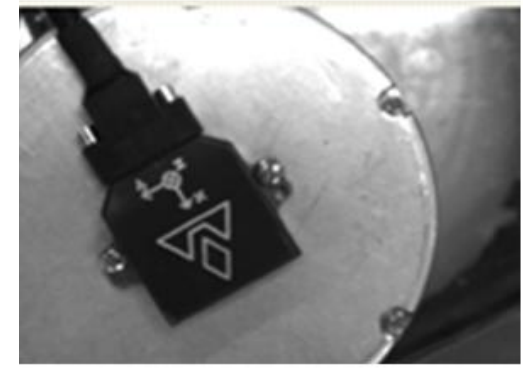

Step 1: Image target with gamma camera while imaging the top of the gamma camera with visible camera

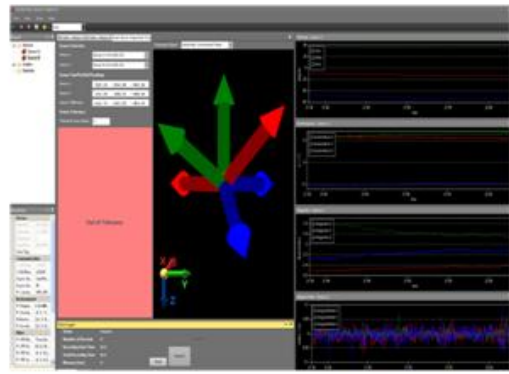

Step 2: Using the information from the AHRS units, align the two cameras

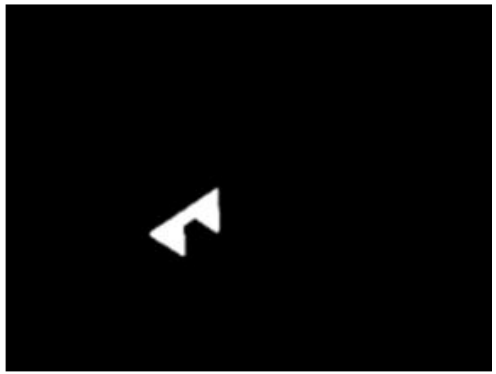

Step 3: Segment the Vector Nav logo and find its center and area

Figure 29: OffSet Correction USing the logo of the AHRS Unit on the gamma CAMERA

The gamma camera has a parallel hole collimator. It images incident rays that are parallel to the collimator walls, resulting in a constant pixel density in terms of pixels $/ \mathrm{mm}^{2}$. The pixel density value for the visible camera depends on the working distance i.e., the distance between the target and the lens of the camera. So the relative scaling factor between the two images is a function of the working distance. The area of the segmented AHRS logo is calculated in pixels. Its area in $\mathrm{mm}^{2}$ is a constant known value. These can be used to find the pixel density (pixels/ $\mathrm{mm}^{2}$ ) of the visible image. Dividing this by the pixel density of the gamma image gives the relative scaling factor.

The final gamma image containing only the selected outlines of the ROIs is rotated, and the translational and scaling offsets are corrected., As a result, both images have the same FOV, scaling and are centered at the same pixel location. However, the size of the gamma image is 270 $\mathrm{x} 270$ whereas the size of the visible image is $756 \times 1024$. To correct for the difference in sizes, the images are first centered, and then zero padding is performed on the gamma image. Finally, 
the gamma image is automatically saved in a pre-specified location. LabVIEW reads the gamma from this location and overlays it on the visible/ NIRf image.

\subsection{Image Co-registration Walk Though}

The complete process of gamma image segmentation and co-registration with the visible/NIRf image is performed in a gelatin phantom experiment. The process is explained step by step in the following section.

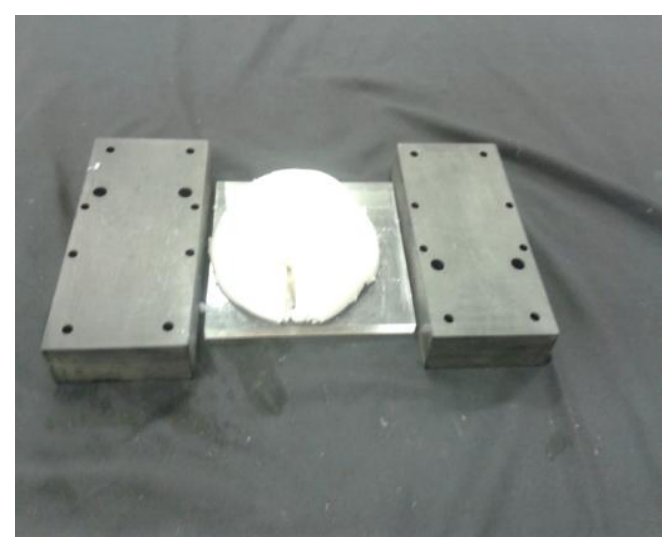

Step 1: An opaque gelatin phantom containing two simulated $8.3 \mathrm{~mm}$ diameter targets containing solutions of ${ }^{99 \mathrm{~m}} \mathrm{Tc}$ and Indocyanine green (ICG) was created.

Figure 30: Photograph of the opaQue gelatin PHANTOM PRIOR TO SURGERY

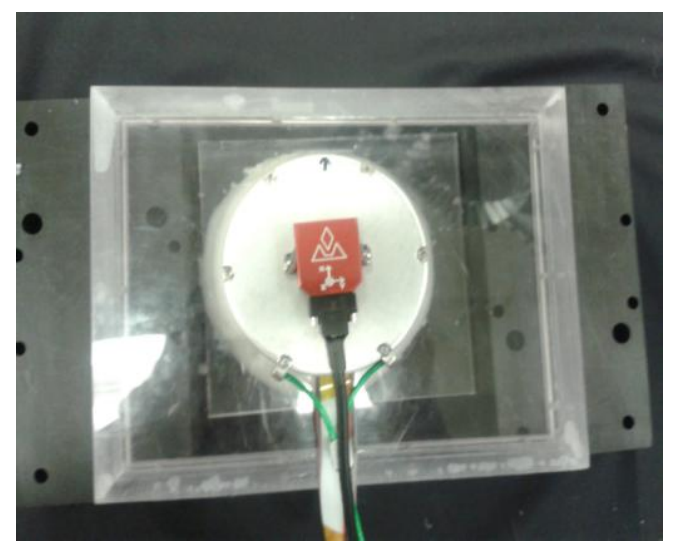

Step 2: The phantom is imaged with the gamma camera for 30-60 seconds

FIgURE 31: VIDEO FRAME OF GAMMA CAMERA DURING GAMMA IMAGE ACQUISITION

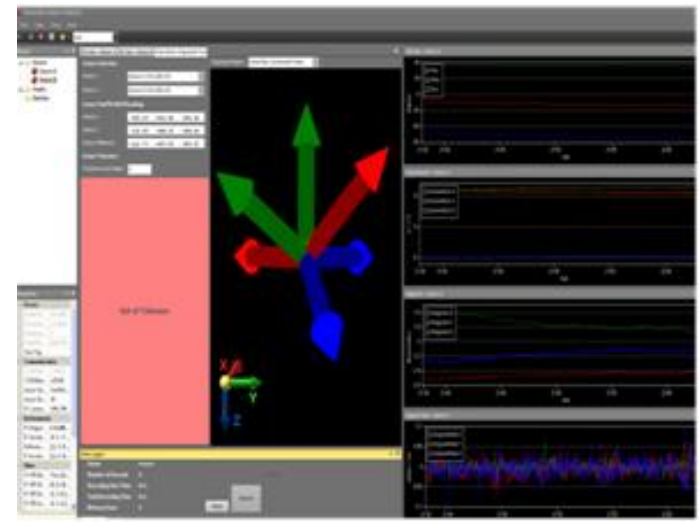

Step 3: While the gamma camera is acquiring the image, align the two cameras using the VectorNav software. Figure 32: CONTROL MONITOR SHOWING THE ORIENTATIONS OF THE TWO AHRS UNITS 


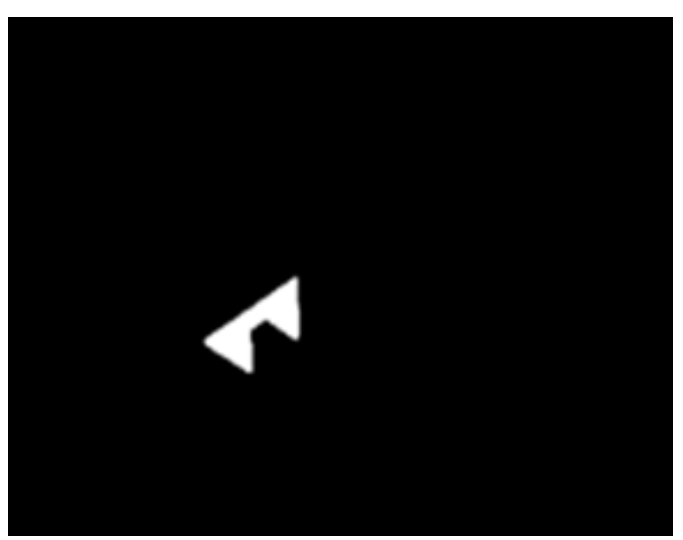

Step 4: Segment the VectorNav logo on the AHRS unit to give the offset corrections

Figure 33: BINARY IMAGE OF GAMMA CAMERA AHRS LOGO, USED TO CALCULATE ROTATIONAL, TRANSLATIONAL, AND SCALING OFFSETS

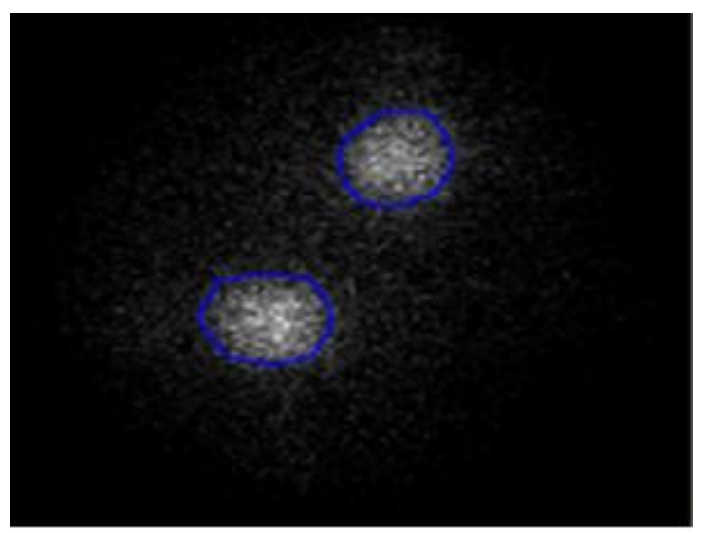

Step 5: Segment the gamma image and select the desired ROIs. Then perform the offset corrections.

Figure 34: ORIGINAL GAMMA IMAGE SHOWING THE TWO TARGETS AND THEIR SEGMENTED ROIS, BEFORE ROTATIONAL AND TRANSLATIONAL TRANSFORMATION

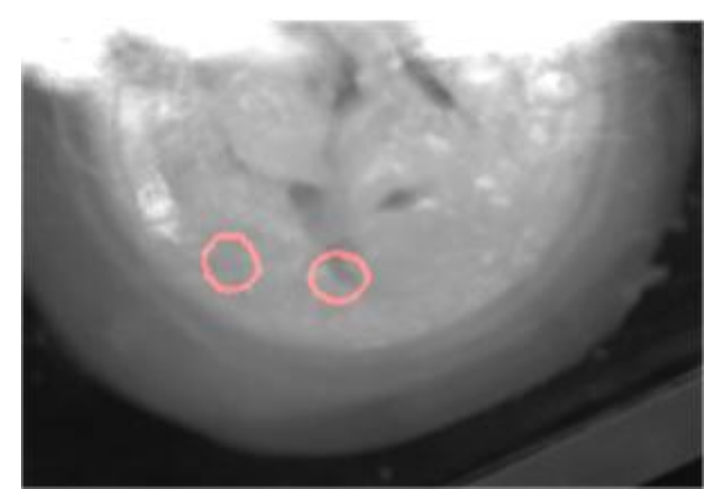

Step 6: Overlay the corrected gamma outlines on the visible/NIRf image to initially guide the incision

FiguRE 35: VISIBLE FRAME WITH TRANSFORMED ROIS OVERLAID

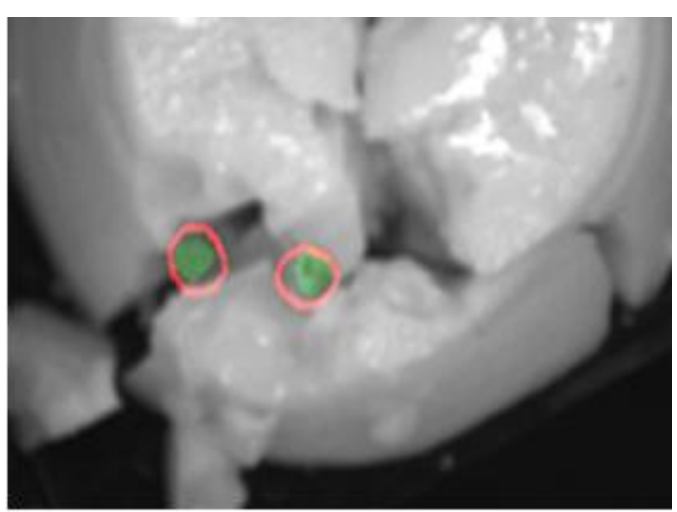

Step 7: Perform the incision until the NIR fluorescence is visible. Then use NIRf for precise visualization of tumor margins.

Figure 36: Fused visible/NIRf image with gamma ROIs 
The method used for image segmentation and co-registration is summarized in the following flow chart:
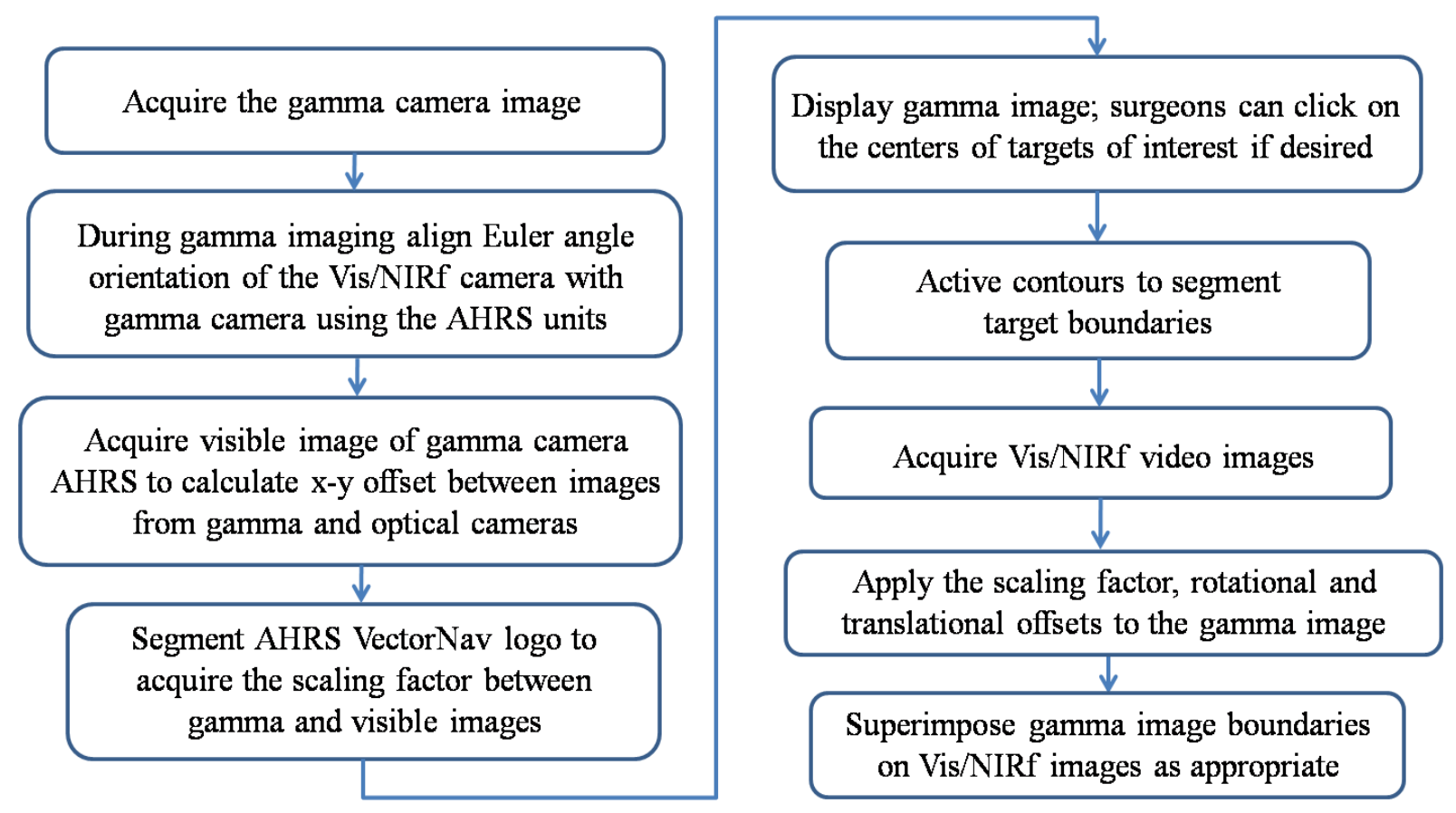

Figure 37: Flowchart of Image Segmentation and Co-registration processes 


\section{CHAPTER 5: EXPERIMENTAL RESULTS}

The IMIS must be capable of accurately localizing the target initially, calculating the number of counts in each ROI and co-registering the images. A series of tests were performed to evaluate the accuracy of the program for each of these tasks. Additionally, tests were conducted to estimate the repeatability of the program.

\subsection{Repeatability of the Gamma Image Segmentation}

The repeatability of the gamma image segmentation program was measured using a set of three hollow spherical targets. The lesions had inner diameters of $6.1,8.3$ and $12 \mathrm{~mm}$, filled with a mixture of $18.9 \mu \mathrm{mol} / \mathrm{L} \mathrm{ICG}$ solution and ${ }^{99 \mathrm{~m}} \mathrm{Tc}$-pertechnetate resulting in activities of $0.63,0.81$ and 1.5 MBq, respectively. Five consecutive images of the targets were obtained without repositioning the cameras or the targets. The pixel location of the center of each lesion was calculated. Determination of the location of the centers of the ROIs was repeatable with a mean error of 0.5 pixels. The number of gamma counts in each ROI was repeatable with a standard deviation of $4 \%$ from the mean. The same set of lesions was imaged by removing and repositioning the camera before each acquisition. Then, the distance between any two lesion centers was calculated. It was reproducible with a mean error of 0.8 pixels.

\subsection{Calculating the Number of Counts}

The standard guideline in SLNb dictates that the surgeon removes all lymph nodes from the patient whose gamma probe count rate in-vivo exceeds one tenth of the count rate of the hottest lymph node ex-vivo. These count rate measurements are done with a non-imaging surgical probe that generally has a wide viewing angle. The combination of the large size of excised lymph 
nodes compared to the size of the pinhole aperture in non-imaging probe collimators, and the strong dependence of non-imaging probe sensitivity with changing probe-to-source separation means that the excised node activity measured using the probe can be inaccurate. Furthermore, operator dependence was observed during a clinical study (MEL54) when comparing a surgeon's and a resident's gamma measured probe count rates of the same nodes (1). This problem is resolved by using the gamma camera and the segmentation algorithm to have reliable and consistent real-time count rate information in the OR. The following table shows the count data for the ex-vivo nodes from the MEL54 study acquired by using the gamma probe compared to the segmentation algorithm and the gamma camera (second and third columns). To account for the difference in acquisition times and sensitivity, the results are reported in terms of calculated activity.

\begin{tabular}{|c|c|c|c|c|}
\hline Patient & Gamma probe count & Gamma camera & Gamma probe & Gamma camera \\
number & $(10$ seconds $)$ & count $(60$ seconds $)$ & activity [kBq] & activity [kBq] \\
\hline 4 & 21315 & 4869 & 92.7 & 184.3 \\
\hline 4 & 3292 & 495 & 14.3 & 18.7 \\
\hline 6 & 23965 & 4545 & 104.2 & 172.0 \\
\hline 7 & 1459 & 297 & 6.3 & 11.2 \\
\hline 7 & 2768 & 405 & 12.0 & 38.2 \\
\hline 8 & 5255 & 1008 & 22.8 & 34.4 \\
\hline 8 & 3911 & 909 & 17.0 & 10.2 \\
\hline 8 & 2131 & 270 & 9.3 & 5.5 \\
\hline 8 & 1402 & 144 & 6.1 & 6.8 \\
\hline 8 & 1359 & 216 & 5.9 & 7.5 \\
\hline 8 & 1721 & 180 & & \\
\hline
\end{tabular}




\begin{tabular}{|c|c|c|c|c|}
\hline 9 & 19573 & 4518 & 85.1 & 171.0 \\
\hline 9 & 2872 & 1350 & 12.5 & 51.1 \\
\hline 9 & 3919 & 2061 & 17.0 & 78.0 \\
\hline 9 & 3646 & 1890 & 15.9 & 71.5 \\
\hline 10 & 8152 & 3627 & 35.4 & 137.3 \\
\hline 11 & 15133 & 2358 & 65.8 & 89.3 \\
\hline 11 & 17794 & 2835 & 77.4 & 107.3 \\
\hline 11 & 2583 & 162 & 11.2 & 6.1 \\
\hline 11 & 8283 & 783 & 36.0 & 29.6 \\
\hline 12 & 2496 & 297 & 10.9 & 11.2 \\
\hline 12 & 6416 & 1242 & 27.9 & 47.0 \\
\hline 12 & 1922 & 171 & 8.4 & 6.5 \\
\hline 13 & 7702 & 1251 & 33.5 & 47.4 \\
\hline 14 & 4387 & 3168 & 19.1 & 119.9 \\
\hline 14 & 29269 & 3960 & 127.3 & 149.9 \\
\hline 15 & 3734 & 450 & 16.2 & 17.0 \\
\hline 15 & 19978 & 4410 & 86.9 & 166.9 \\
\hline 15 & 10756 & 1620 & 46.8 & 61.3 \\
\hline 17 & 5146 & 1431 & 22.4 & 54.2 \\
\hline 17 & 5443 & 1323 & 23.7 & 50.1 \\
\hline 17 & 2422 & 216 & 10.5 & 8.2 \\
\hline 17 & 1209 & 63 & 5.3 & 2.4 \\
\hline 18 & 11474 & 6516 & 49.9 & 246.7 \\
\hline 18 & 2526 & 414 & 11.0 & 15.7 \\
\hline 18 & 2689 & 360 & 11.7 & 13.6 \\
\hline
\end{tabular}




\begin{tabular}{|c|c|c|c|c|}
\hline 18 & 4695 & 495 & 20.4 & 18.7 \\
\hline 19 & 1217 & 36 & 5.3 & 1.4 \\
\hline 19 & 4806 & 783 & 20.9 & 29.6 \\
\hline 19 & 19049 & 2889 & 82.8 & 109.4 \\
\hline 19 & 5319 & 1017 & 23.1 & 38.5 \\
\hline 21 & 54170 & 9315 & 235.5 & 352.6 \\
\hline 21 & 32093 & 5013 & 139.5 & 189.8 \\
\hline 21 & 17063 & 765 & 74.2 & 29.0 \\
\hline 22 & 52727 & 7749 & 229.2 & 293.3 \\
\hline 22 & 43902 & 5490 & 190.9 & 207.8 \\
\hline
\end{tabular}

TABle 1: Comparison of The MEL54 data From EX-Vivo SLN COUNTS From GAMMA CAMERA AND GAMMA PROBE

It is observed that the activity measured by the gamma probe and the gamma camera do not follow the same trend. To verify the claim that the gamma camera reduces the uncertainties associated with the non-imaging probe, the linearity of the number of counts calculated with respect to varying activity and volume was tested. The experimental setup is shown below.

\subsubsection{Linearity of Counts With Respect to Volume}

For this test, three well plates with 96 wells each were used. A well plate is shown in Figure 38 a) below. Eight of those wells were chosen such that the distance between any two wells is large enough to have only one well in the FOV of the gamma camera for each acquisition. A solution of ${ }^{99 \mathrm{~m}} \mathrm{Tc}$ with activity of $122 \mu \mathrm{Ci}$ in $1.5 \mathrm{~mL}$ was distributed in varying volumes in each of the chosen wells. The volume was measured using a standard micropipette show in Figure 38b). 
The volume range was $40-200 \mu \mathrm{L}$. The concentration of activity $(\mathrm{kBq} / \mathrm{mL})$ was kept constant for all the samples, and hence, the total activity increased linearly with volume. Therefore, the number of counts is expected to increase linearly with volume. From the graph shown below in figure 37 , it is observed that the values follow the expected trend. A fairly linear graph is obtained in the given range. The activity was measured with a mean error of $23.2 \pm 7.1 \%$.

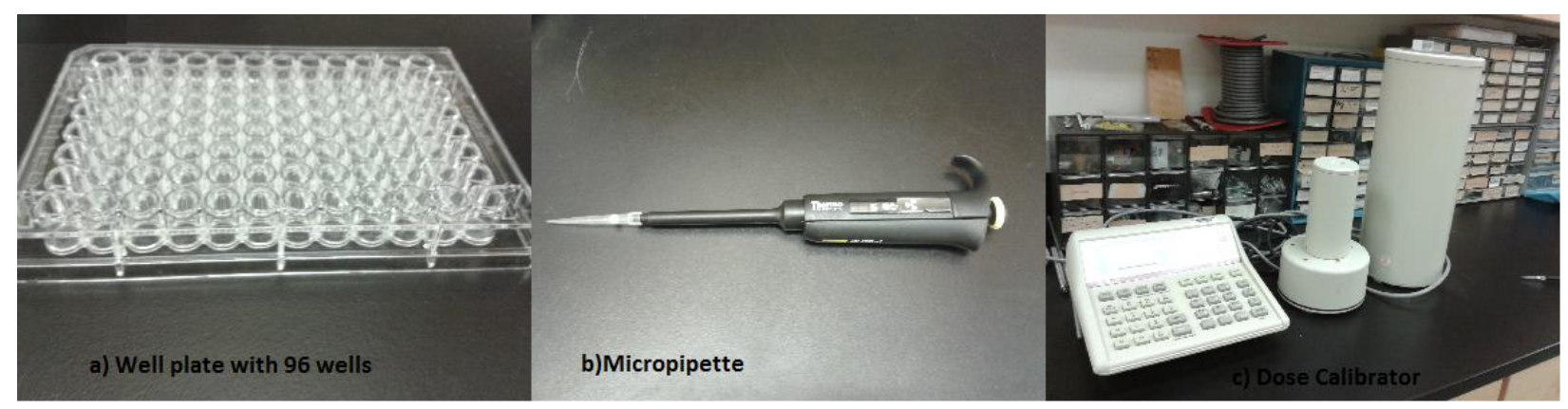

Figure 38: Experimental Setup

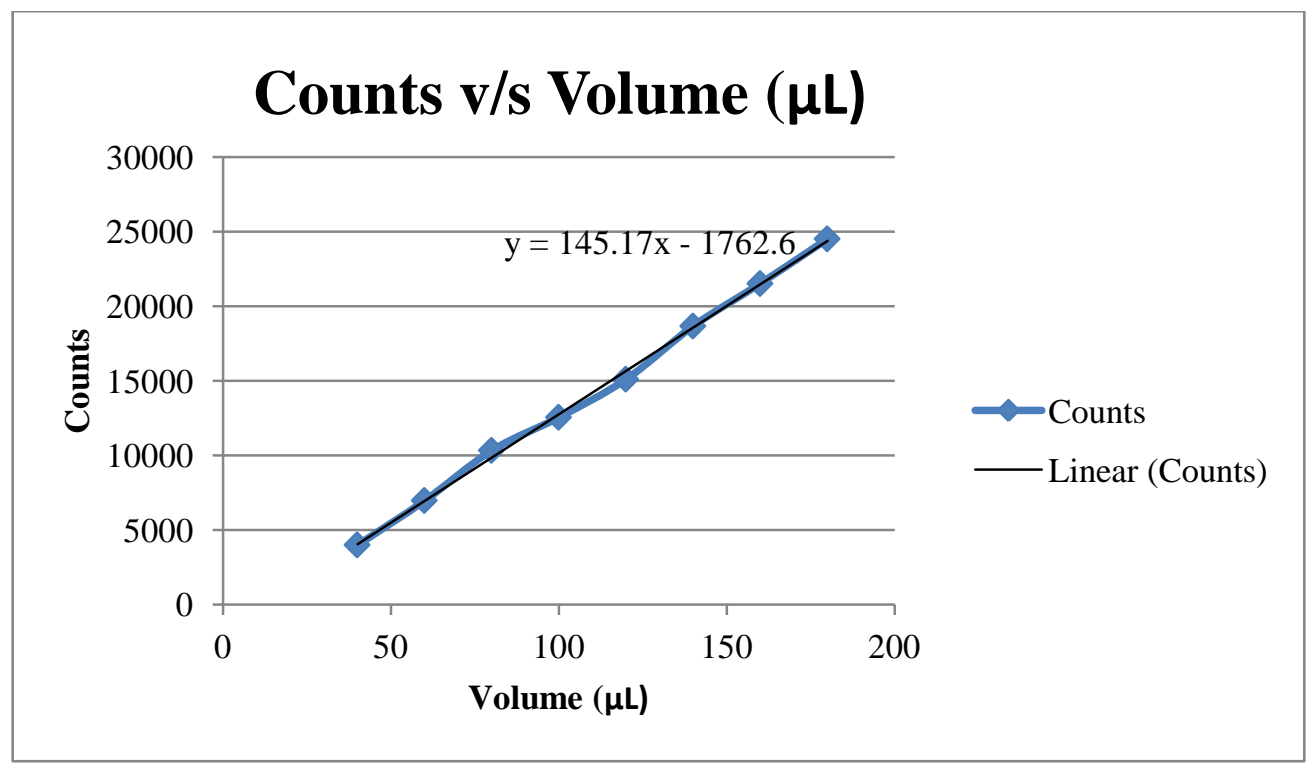

Figure 39: GRAPH OF THE NUMBER OF COUNTS WITH RESPECT TO INCREASING VOLUME OF ISOTOPE 


\subsubsection{Linearity of Counts With Respect to Activity}

The set up is similar to the one described above for measuring the linearity of the count rate measurement. Three well plates with 96 wells each were used. Twelve of those wells were chosen such that the distance between any two wells is large enough to have only one well in the FOV of the gamma camera for each acquisition. The wells were filled with $250 \mu \mathrm{L}{ }^{99 \mathrm{~m}} \mathrm{Tc}$ of varying activity. The volume was kept constant for all the samples but the activity per volume was varied. The activity was in the range of $0.98 \mu \mathrm{Ci}$ to $24 \mu \mathrm{Ci}$, which covers the entire range of values seen in the OR. The activity was measured using a dose calibrator shown in Figure $38 \mathrm{c}$ ). The activity in the micropipette was measured before and after squirting the solution into the well. The difference is calculated as the activity of the isotope in the well. Again, the number of counts is expected to vary linearly with activity. From the graph shown below in Figure 38, it can be seen that the values follow the expected trend. A fairly linear graph is obtained. The activity was measured with a mean error of $27.23 \pm 3.9 \%$. From these tests, it can be concluded that the gamma camera is capable of giving reliable information for the relative number of counts between the hottest excised node and the un-excised nodes.

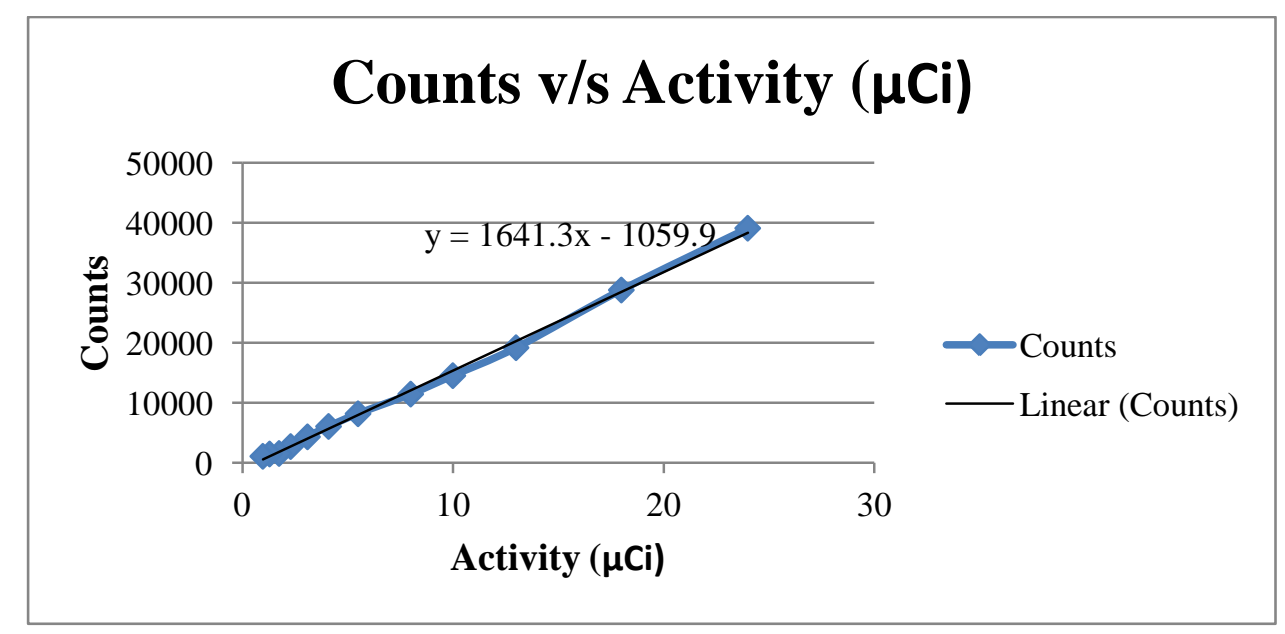

FIgURE 40: GRAPH OF THE NUMBER OF COUNTS WITH RESPECT TO INCREASING ACTIVITY OF ISOTOPE 


\subsection{Repeatability of the Full Process (Segmentation and Co-registration)}

The overall repeatability program was measured using the same set of three hollow spherical targets. The lesions had inner diameters of $6.1,8.3$ and $12 \mathrm{~mm}$ and were filled with a mixture of $18.9 \mu \mathrm{mol} / \mathrm{L}$ of ICG solution and ${ }^{99 \mathrm{~m}} \mathrm{Tc}-$ pertechnetate resulting in activities of $0.63,0.81$ and 1.5 MBq, respectively. First, the lesions were imaged with the gamma camera. Then the back of the gamma was imaged. Then the gamma camera was removed from the FOV and the visible/NIRf image was obtained. Then the gamma camera was placed back in approximately the same position, and the process was repeated five times. This experiment aims to determine how well the algorithm was able to consistently and accurately determine the centers of the lesions and coregister the gamma and visible/NIRf images. The pixel location of the center of each lesion is calculated both in the final corrected gamma image and in the fused visible/NIRf image. The distance between the center of an ROI in the gamma image and the corresponding center in the NIRf image was repeatable with a mean error of $1.2 \pm 0.58 \mathrm{~mm}$. The distance between the centers of any two ROIs in the gamma image and the corresponding distance in the NIRf image was repeatable with standard deviation of $7.64 \%$ from the mean. The number of gamma counts in each ROI was repeatable with a mean error of $10.73 \pm 5.4 \%$.

\subsection{Comparison of the Gamma Image Segmentation with a Local Threshold Based Segmentation}

One of the main concerns with the current segmentation algorithm was that it uses a global threshold for binarization. Small regions of local high contrast (as in the case of deep lesions) could potentially be undetected with global thresholding. To validate this algorithm, its 
performance has been compared to that of a local threshold based segmentation program. This program uses two thresholds, high and low. Initially, the high threshold is applied and all the regions with a high intensity gradient are detected. Then, this image (with high gradients) is subtracted from the original image and a low threshold is applied to detect any small areas of high local contrast that could have been obscured by the large areas of high global contrast.

Both programs were used on the clinical data from Mel54 study. The current gamma image segmentation algorithm was discovered to be perform better than the local thresholding algorithm because:

a) Both the global and local thresholds have to be set for each case. It goes against the goal of minimizing user interaction.

b) The best results are seen for a local threshold of 0 which is the same as using a single global threshold.

c) It lacks the flexibility of letting the surgeon choose which ROIs to see on the final image

d) Since the IMIS is an intraoperative system and not a diagnostic one, it is not necessary to be able to clearly identify all the nodes at once. Once the hottest nodes are identified and excised, the patient is re-imaged. The deep set nodes (small regions of local high contrast) would then become visible.

e) The main drawback of the local threshold based program is that it gives a significant number of false positives with the setting that is required to view deep set nodes. An example is shown below in figure 39 . 


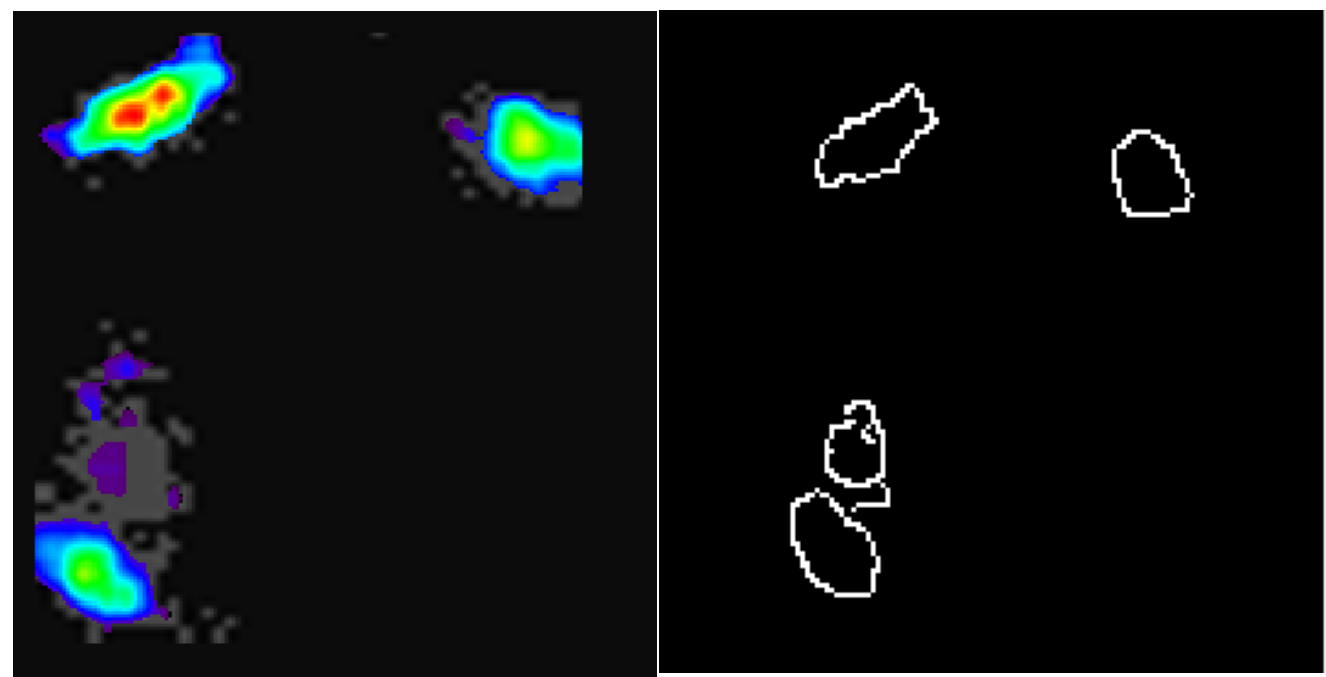

FIGURE 41: LOCAL THRESHOLD BASED SEGMENTATION (LEFT) COMPARED TO CURRENT METHOD (RIGHT)

Both the algorithms were tested on 5 random images from the MEL 54 study. The number of nodes is considered to be metric of interest. The number of nodes detected by each program is compared to the actual number of nodes. The current program is called program 1 and the local threshold program is called program 2. We consider two cases for program 2- number of nodes detected by default threshold values and number of nodes detected by a local threshold of 0 . It can be concluded that program 1 and program 2 with a local threshold of 0 show comparable performance. Program 2 with the default threshold values gives a high number of false positives.

\begin{tabular}{lllll}
\hline Patient & $\begin{array}{l}\text { Actual } \\
\text { Number of } \\
\text { Nodes }\end{array}$ & $\begin{array}{l}\text { Number of nodes } \\
\text { detected by } \\
\text { program } 1\end{array}$ & $\begin{array}{l}\text { Number of nodes } \\
\text { detected by local } \\
\text { threshold program for } \\
\text { default threshold values }\end{array}$ & $\begin{array}{l}\text { Number of nodes } \\
\text { detected by program } \\
\text { threshold=0 }\end{array}$
\end{tabular}

\begin{tabular}{lllll}
\hline $\mathbf{4}$ & 1 & 1 & 1 & 1 \\
$\mathbf{6}$ & 1 & 1 & 1 & 1 \\
$\mathbf{1 0}$ & 5 & 4 & 8 & 4 \\
$\mathbf{1 3}$ & 2 & 2 & 4 & 3 \\
$\mathbf{1 5}$ & 2 & 2 & 5 & 2 \\
\hline
\end{tabular}

TABLE 2: COMPARISON OF THE NUMBER OF NODES DETECTED BY EACH SEGMENTATION PROGRAM 


\subsection{Sensitivity of the Segmentation Program to Changes in the Target Parameters}

In the present context, sensitivity is defined as the change in a test parameter with respect to a change in some property of the target. To test for the sensitivity of the segmentation program, four target parameters are considered- lesion size, depth, activity and volume. Diameter of the lesion is taken as the test parameter.

\subsubsection{Sensitivity With Respect to Volume}

The experimental set up is similar to the one described in sections 5.2.1 and 5.2.2. A well plate with 96 wells is taken. Eight of the wells were chosen such that only one of them is in the FOV of the gamma camera for each image acquisition. A solution of ${ }^{99 \mathrm{~m}} \mathrm{Tc}$ with activity of $122 \mu \mathrm{Ci}$ in $1.5 \mathrm{~mL}$ was prepared and distributed in varying volumes in each of the chosen wells. The volume range was $40-200 \mu \mathrm{L}$. Since the concentration of activity $(\mu \mathrm{Ci} / \mu \mathrm{L})$ is constant, the total activity in the well depends on the volume of the solution present in it.

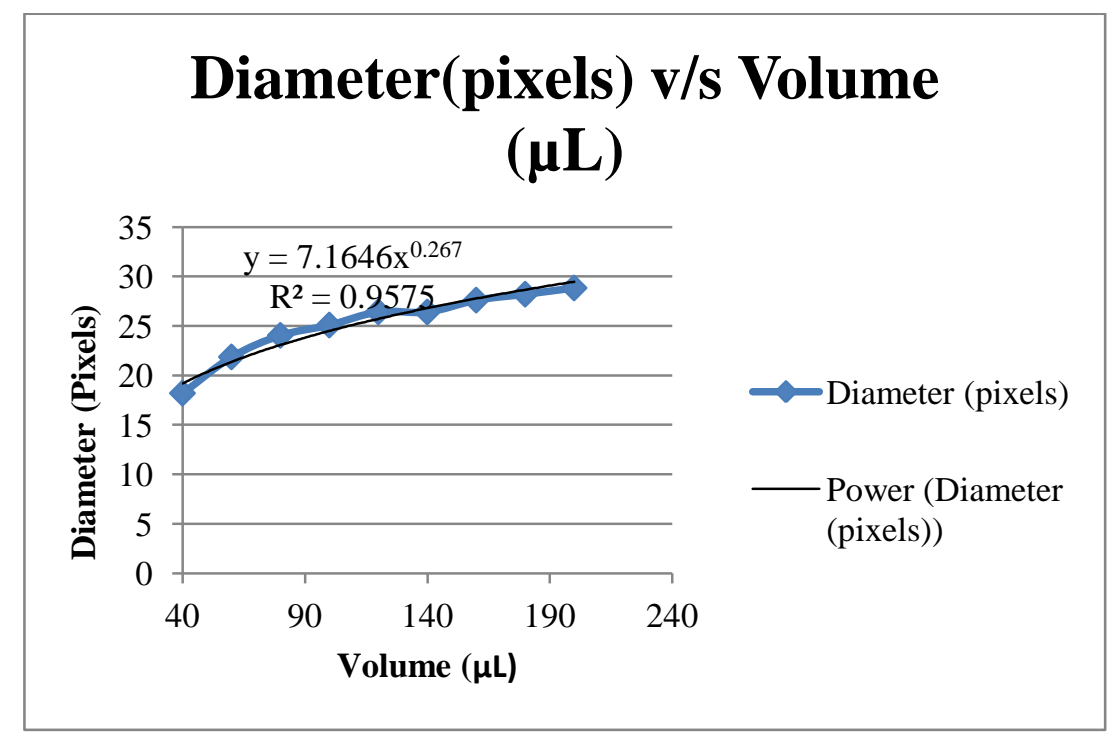

Figure 42: GRAPH OF DIAMETER OF TARGETS OF SAME ACTIVITY BUT VARYING VOLUME 
An image of each of the 8 chosen wells is acquired and the diameter of the target calculated in pixels. The above graph shows the diameter of the target in the gamma image measured in pixels with respect to the volume. A trend line with a power fit is also shown. It has to be noted that the area of cross section of all the targets is the same. Hence, it can be concluded that the diameter of the target in the gamma image increases proportionally to an increase in volume even if the cross sectional area remains constant.

\subsubsection{Sensitivity With Respect to Activity}

In a similar well plate with 96 wells, twelve wells were filled with the same volume $(250 \mu \mathrm{L})$ of ${ }^{99 \mathrm{~m}} \mathrm{Tc}$ with varying activity. The activity was in the range of $0.98 \mu \mathrm{Ci}$ to $24 \mu \mathrm{Ci}$. The cross sectional area of the wells is constant.

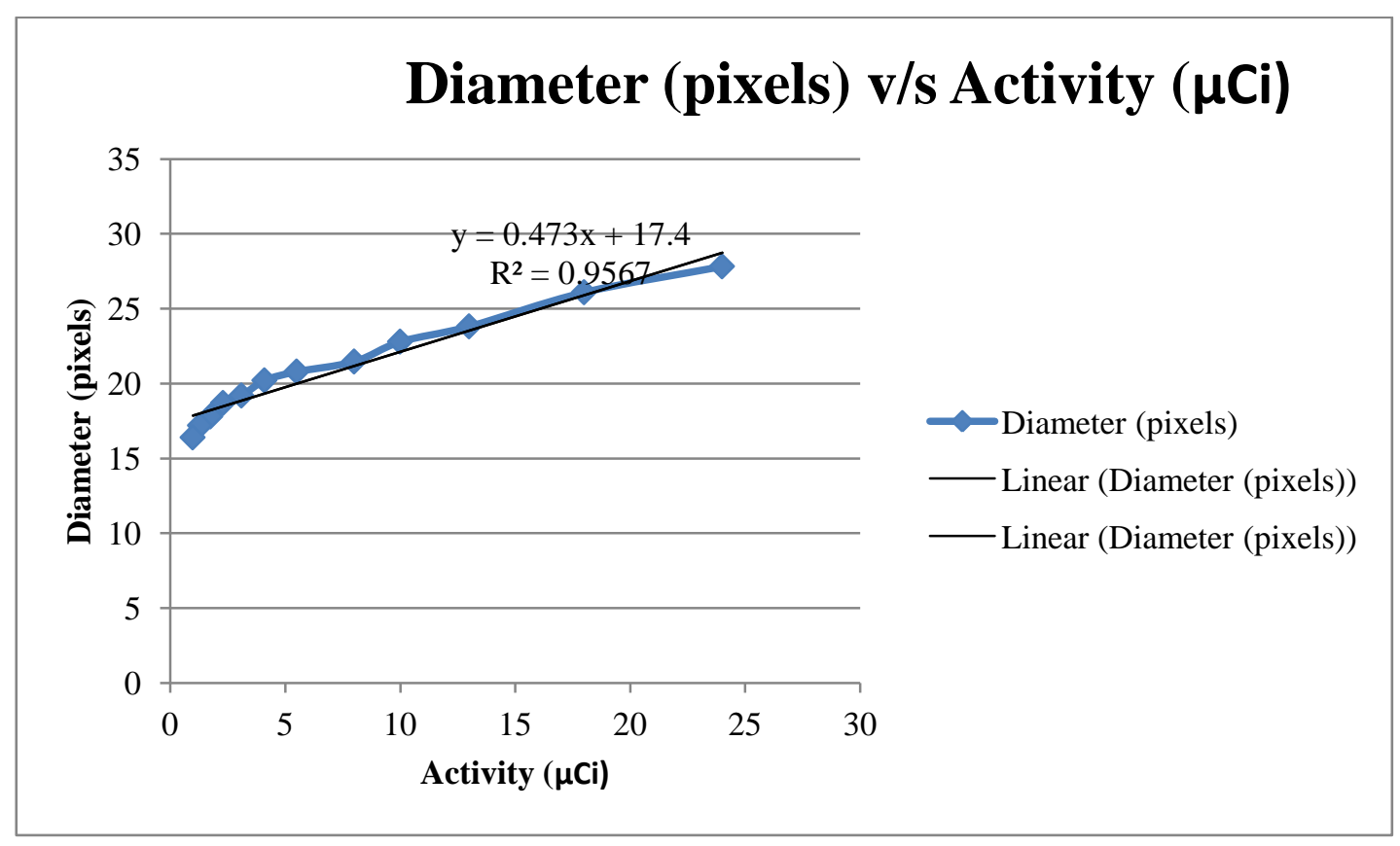

Figure 43: GRAPH OF THE DIAMETER OF A TARGET WITH RESPECT TO VARYING ACTIVITY 
An image with the gamma camera was acquired for each of the wells. The diameter of each well was calculated. The above graph shows the diameter of the lesion in pixels with respect to varying activity. It can be seen that the diameter of the target in the gamma image varies linearly with the activity of the target even if the cross sectional area remains constant.

\subsubsection{Sensitivity With Respect to Lesion Size and Depth}

To calculate the sensitivity of the program with respect to lesion size and depth, five lesions of diameters $6 \mathrm{~mm}, 6.9 \mathrm{~mm}, 8.1 \mathrm{~mm}, 9.8 \mathrm{~mm}$ and $12.9 \mathrm{~mm}$ were filled with same activity concentration (activity/unit volume) of ${ }^{99 \mathrm{~m}} \mathrm{Tc}$. Each one was placed at varying depths, in steps of $2 \mathrm{~cm}$, in a water-filled box phantom.

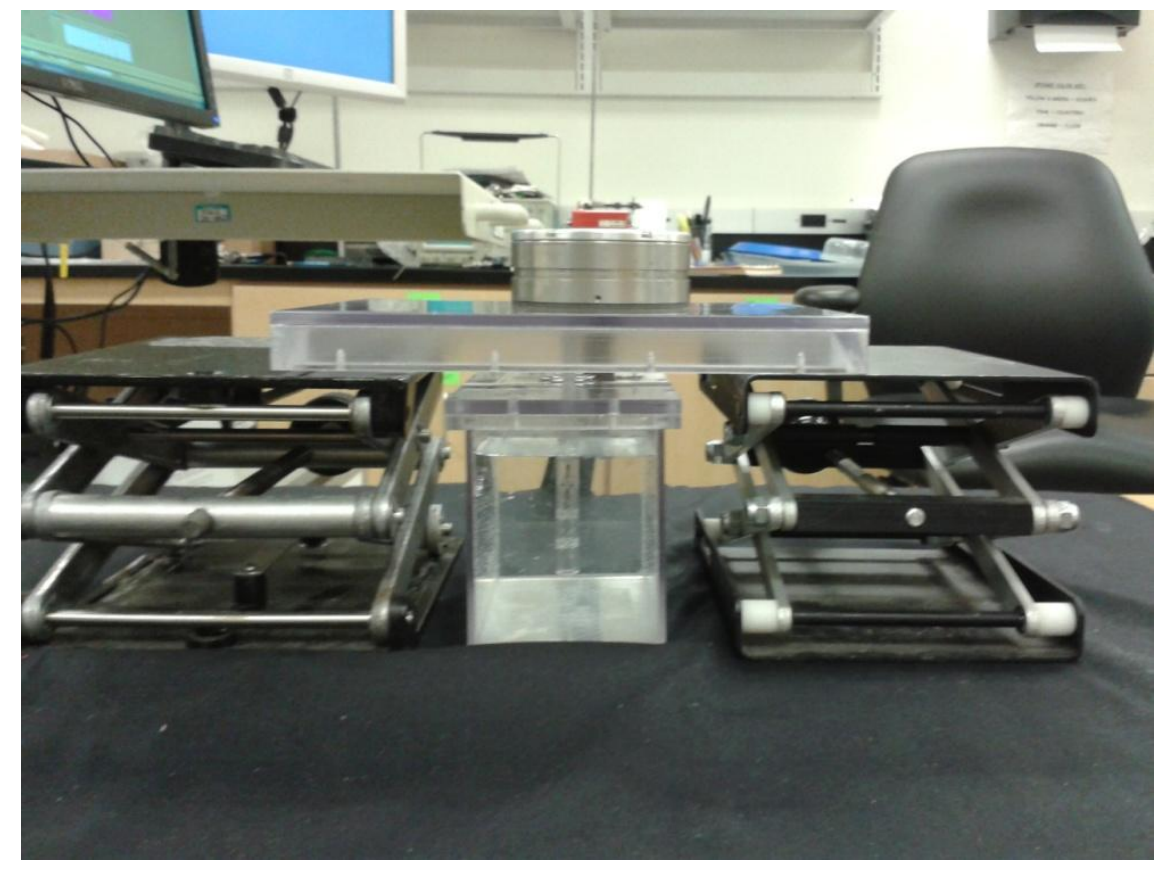

Figure 44: SETUP OF THE EXPERIMENT TO MEASURE SENSITIVITY WITH RESPECT TO LESION SIZE AND DEPTH 
This setup simulated the effect of lesions being at different depths inside the body with the gamma camera placed close to the surface. The gamma radiation being emitted from deeper nodes has to pass through a longer distance in the medium, adding scatter and attenuation. In the first step, the diameter of the lesion was measured in the gamma image in pixels and was plotted against the actual diameter in millimeters. Figure 43 shows the linear relationship.

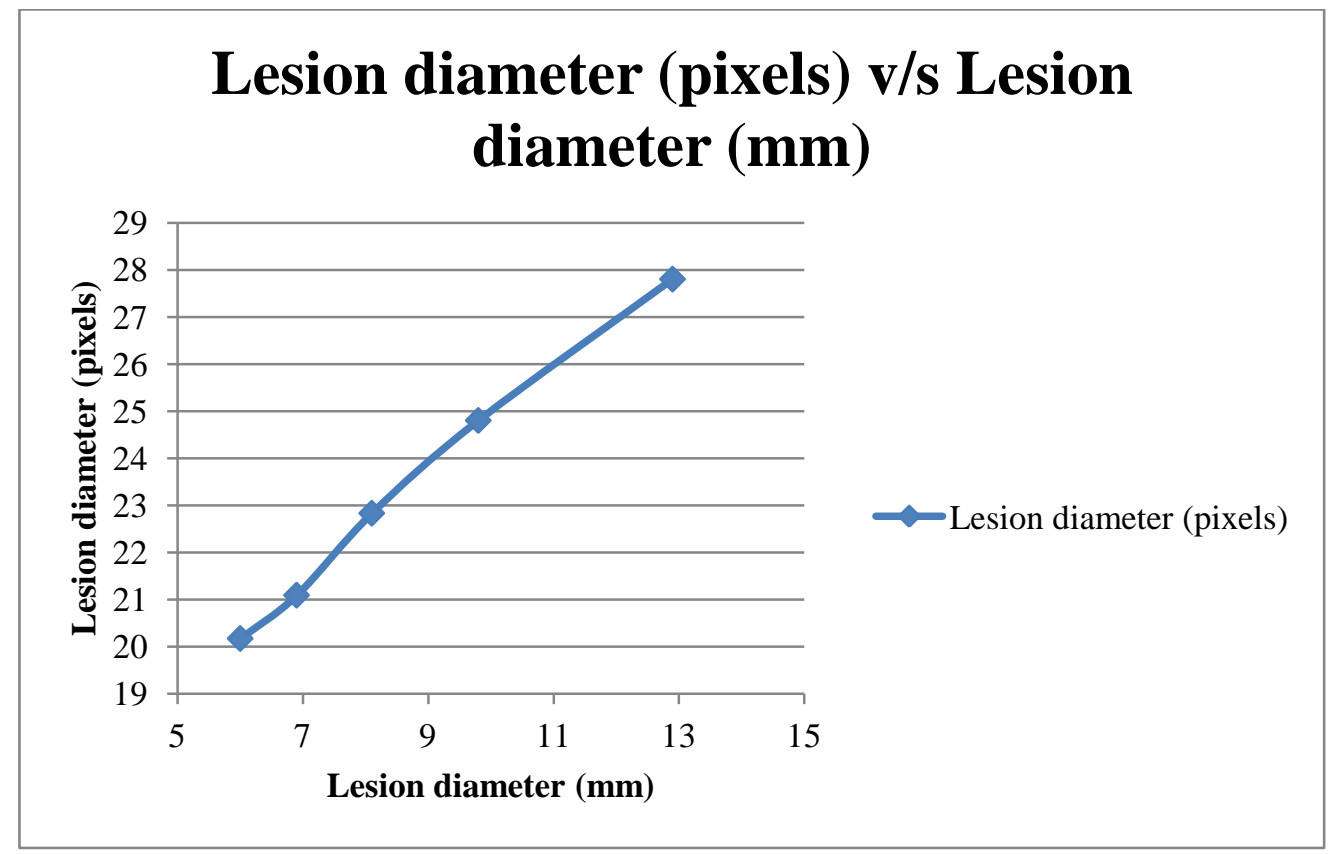

Figure 45: GraPh OF DiAMETER OF LESION IN GAMMA IMAGE WiTH RESPECT TO ACTUAL DiAMETER

In the next step, the diameter measured in the gamma image is plotted with respect to the depth of the scattering material (water). This set of data is more variable compared to the other data sets because an increase in depth decreases the quality of the images due to attenuation and scatter. Hence the images become more difficult to segment. The plots for all the lesion sizes follow the same trend as seen in figure 44. As the depth increases, the diameters of the lesions in 
the images increases (image blurring/distortion). Eventually, the blur became large enough to obscure the image boundaries. This point was reached at lesser depths for smaller lesions(lesions 1,2 and 3) and at larger depths for larger lesions(lesions 4 and 5).

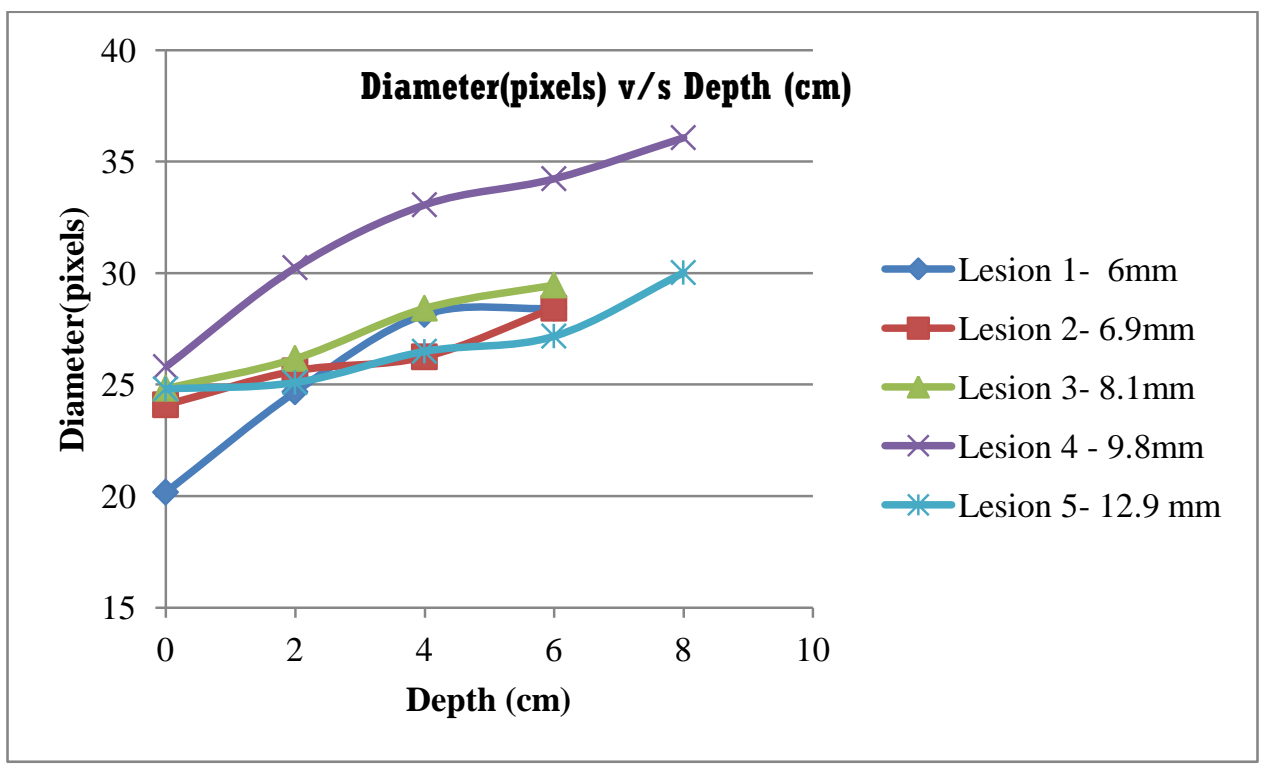

FIgURE 46: GRAPH OF LESION DIAMETER WITH RESPECT TO DEPTH 


\section{CHAPTER 6: DISCUSSION/CONCLUSION}

\subsection{Discussion}

A literature study was done to compare the relative advantages and disadvantages of using only NIRf imaging and of combining gamma imaging. Most of the current applications of NIRf only imaging were in fields that do not require target localization, such as tissue graft quality testing and cervical cancer surgery (18) (19). One of the main NIRf systems was reported by DeGrand (20). It is called fluorescence-assisted resection and exploration (FLARE) system. The system has been used in animal model trials $(20 ; 21 ; 22 ; 23 ; 24)$ and human clinical trials including breast cancer SLNB (25; 26), ex-vivo colorectal SLN mapping (24) head and neck cancer SLNB (27) and cervical cancer SLN mapping (28). Handa (29) reported development of a method of NIRF imaging, that was used in intraoperative graft assessment as well as fluorescence guided SLNB in animal models (30). A commercial NIRF imaging system, Novadaq Spy was used in various clinical medical imaging applications, including coronary and graft imaging $(\mathbf{3 1} ; \mathbf{3 2})$ organ transplantation (33) and other cardiac function assessment procedures (34). One group explored the possibility of using Gamma and NIRf imaging as complementary modalities and concluded that it would give better results than NIRf alone (35).

Gamma image acquisition usually takes $30-60$ seconds. The hypothesis is that it would be possible to align the two cameras and image the back of the gamma camera within this time. To verify this claim, seven volunteers were asked to perform the alignment and imaging of the back of the gamma camera and they were timed and the procedure was repeated 5 times. In most cases, the process was completed in less than 30 seconds. The time taken did not exceed the limit 
of 60 seconds, even for the outliers. These results are promising and illustrate that even without extensive training, surgeons would be able to successfully align the camera during the gamma image acquisition.

The segmentation of the logo on the AHRS unit and the gamma image segmentation were both timed. To measure what would realistically be obtained, all the programs which are usually used in the OR (KMAX, LAbVIEW, VectorNav) were running simultaneously with the segmentation algorithm.

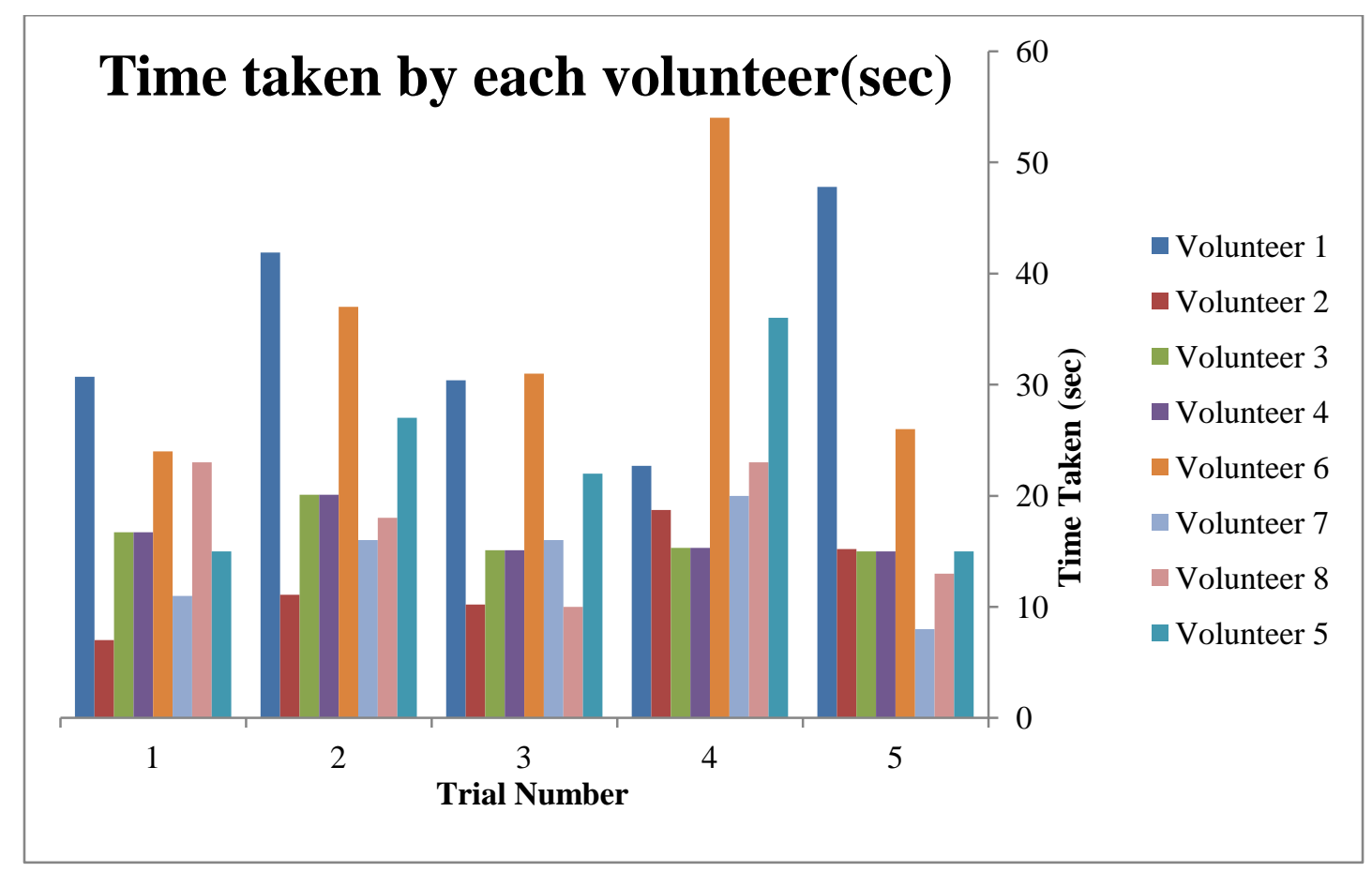

Figure 47: Time TAKen to ALIGN THE CAMERAS AND IMAgE BACK OF THE GAMMA CAMERA

The segmentation initializes and runs each snake sequentially. It can be modified to do parallel processing. However, given the fact that the IMIS only has a dual core processor, and three other computationally intensive programs are running at the same time, serial processing would work best. 


\begin{tabular}{lll}
\hline Time taken for AHRS logo & Time taken for one node & Time taken for two node \\
\hline segmentation (secs) & segmentation (secs) & segmentation (secs) \\
\hline $\mathbf{0 . 8 2 4 0 6}$ & 3.013876 & 4.208339 \\
\hline $\mathbf{0 . 7 0 4 3 0 3}$ & 3.704303 & 4.283719 \\
\hline $\mathbf{0 . 7 1 0 3 1 2}$ & 3.710312 & 3.954132 \\
\hline $\mathbf{0 . 7 0 3 7 4 4}$ & 3.176413 & 4.913012 \\
\hline $\mathbf{0 . 7 0 3 2 7 1}$ & 3.311317 & 4.664562 \\
\hline $\mathbf{0 . 6 9 6 6 4 9}$ & 3.226145 & 4.390718 \\
\hline $\mathbf{0 . 7 1 6 5 3 8}$ & 3.080468 & 4.34579 \\
\hline $\mathbf{0 . 6 9 2 3 5 1}$ & 2.811527 & 4.243371 \\
\hline $\mathbf{0 . 7 0 0 1 7 9}$ & 2.753473 & 4.735619 \\
\hline $\mathbf{0 . 7 0 5 2 5 9}$ & 3.286948 & 4.516644 \\
\hline
\end{tabular}

TABLE 3: TIME TAKEN BY THE SEGMENTATION PROGRAM

The theoretical feasibility of using the program has been verified. The next step is to verify that the system can practically be used in an OR setting. One of our collaborators Dr Traci Hedrick performed a phantom surgery to check if there were any issues she may run into during a surgery. She first went through the software process, which includes the gamma image acquisition, image segmentation and overlay. Using the information from the gamma image for initial target localization, she incised the phantom until the lesion came close to the surface and NIRf emission was visible. Overall, she felt that the system is easy to use and the programs are 
user friendly. One of our concerns was whether having to keep the AHRS unit in the FOV of the visible camera would be difficult. Dr. Hedrick confirmed that it did not add any difficulty to the image acquisition process. She was able to align the cameras and image the back of the gamma camera in less than 30 seconds.

There were two concerns raised by Dr Hedrick. Presently, only the gamma camera is placed in a sterile drape. The visible/NIRf camera is not sterilized as it was far enough from the patients in the case of melanoma surgery. But, for colo-rectal surgery, it could be closer. So, the camera head has to be placed in a sterile plastic drape. This could potentially affect the segmentation process of the AHRS logo. An experiment was conducted where both the cameras were placed in sterile drapes and the back of the gamma camera was imaged with the visible camera. It was observed that as long as the plastic drape was held taut against the imaging part of the camera, it did not affect the segmentation.

Use of the plastic drapes also causes attenuation of light. Experiments were conducted to quantify the attenuation and identify potential problems. Three types of plastic drapes were used and the percentage drop in light intensity was measured with respect to intensity measured without the drape. The results are summarized below. It can be observed that the attenuation is in the allowable range.

\begin{tabular}{llll}
\hline Type of Light & $\begin{array}{l}\text { Percentage Drop in } \\
\text { Intensity- Cover } \mathbf{1}\end{array}$ & $\begin{array}{l}\text { Percentage Drop in } \\
\text { Intensity - Cover 2 }\end{array}$ & $\begin{array}{l}\text { Percentage Drop in } \\
\text { Intensity- Cover 3 }\end{array}$ \\
\hline Excitation Intensity & $5.2 \pm 0.53$ & $3.12 \pm 0.93$ & $4.3 \pm 1.14$ \\
\hline Emission Intensity & $2.4 \pm 0.8$ & $2.6 \pm 0.6$ & $2.72 \pm 0.95$ \\
\hline RGB Light Intensity & 3.36 & 1.38 & 2.37 \\
\hline
\end{tabular}


The second concern raised by Dr Hedrick was that holding the gamma camera still for a period of 60 seconds might be difficult if the image is to be acquired in a non-horizontal plane. To solve this problem, the possibility of using a mechanical arm which can hold the camera in any direction is being explored.

Overall, it was concluded that the IMIS and the image segmentation and co-registration programs look promising to be used in an OR setting.

\subsection{Conclusion}

A novel method for image segmentation and co-registration of different modalities is discussed in this thesis. The IMIS consists of gamma, NIRf and visible imaging modalities. Gamma rays are detected by a small hand held gamma camera. NIR and visible images are captured by a single dual CCD video camera. Two AHRS units are used to align the two cameras. The logo on the AHRS unit on the back of the gamma camera is used to calculate the offset values between the two images for co-registration. Gamma image segmentation is performed by using filtering, binning, binarization, and Canny edge detection. Finally, snakes are used to give the surgeon the flexibility of choosing particular nodes to be segmented in the final image. One major application that has been explored of the segmentation process is the calculation of the number of gamma ray counts found within a segmented lesion. It has been experimentally shown that the gamma camera, with the segmentation algorithm, gives better performance and more reliable results than the non- imaging gamma probe. The working of the algorithm has been tested in bench top and other phantom experiments. It is shown to give good results for repeatability and sensitivity with respect to target parameters. 


\subsection{Future Directions}

The initial tests for IMIS and the image segmentation and co-registration algorithms have been successfully completed in a laboratory environment. It must now be used in human subjects in clinical trials and any problems that may turn up have to be fixed. A good spatial non-uniformity correction algorithm has to be developed for the gamma camera to minimize the barrel effect that is currently seen in the gamma images. This uniformity correction would give better results in calculating the radioactivity in a given target. A mechanical arm to hold the gamma camera has to be developed to facilitate easy imaging. 


\section{BIBLIOGRAPHY}

1. Popovic, Kosta. Intraoperative Multimodal Imaging System. PhD Dissertation, Department of Physics, University of Virginia, Charlottesville. May, 2013.

2. Positive margins following surgical resection of breast carcinoma: Analysis of pathologic correlates. Miller, Alexander R. 3, 2004 йил 1-June, Journal of Surgical Oncology, Vol. 86, pp. 134-140.

3. Comparison of Mammographically Guided Breast Biopsy Techniques. Velanovich, V. 5, Mау 1999 йил, Annals of Surgery, Vol. 229, pp. 625-630.

4. Patterns of recurrence following a negative sentinel lymph node biopsy in 243 patients with stage I or II melanoma. Gershenwald, Jeffrey E. 6, 1998 йил, Journal of Clinical Oncology, Vol. 16, pp. 2253-2260.

5. The detection and significance of melanoma micrometastases in sentinel nodes. Scolyer, Richard A. 3, 2008 йил September, Surgical Oncology, Vol. 17, pp. 164-175.

6. Lymph Node. Wikipedia. [Online] http://en.wikipedia.org/wiki/Lymph_node.

7. Sentinel Lymph Node. Wikipedia. [Online] http://en.wikipedia.org/wiki/Sentinel_lymph_node.

8. A trend analysis of the relative value of blue dye and isotope localization in 2,000 consecutive cases of sentinel node biopsy for breast cancer. Derossis, A.M., Fey, J., Yeung, H., Yeh, S.D., Heerdt, A.S., Petrek, J., VanZee, K.J., Montgomery, L.L., Borgen, P.I., Cody, H.S. 3rd. 5, 2001 йил November, Journal of the American College of Surgeons, Vol. 193, pp. 473-478.

9. The use of radioisotope combined with isosulfan Blue dye is not superior to radioisotope alone for the identification of sentinel lymph nodes in patients with breast cancer. Bines, S., Kopkash, K., Ali, A., Fogg, L., Wool, N. 4, 2008 йил October, Surgery, Vol. 144, pp. 606-610.

10. SPECT-CT and real-time intraoperative imaging: new tools for sentinel node localization and radioguided surgery? Olmos, R. А. 1, 2009 йил January, European Journal of Nuclear Medicine and Molecular Imaging, Vol. 36, pp. 1-5.

11. Single-Photon Emission Computed Tomography/Computed Tomographyfor Sentinel Node Mapping in Breast Cancer. Husarik, Daniela B. 1, 2007 йил January, Seminars in Nuclear Medicine, Vol. 37, pp. 2933.

12. The hidden sentinel node and SPECT/CT in breast cancer patients. van der Ploeg, М. С. 1, 2009 йил January, European Journal of Nuclear Medicine and Molecular Imaging, Vol. 36, pp. 6-11.

13. [Online] http://en.wikipedia.org/wiki/Data_binning.

14. [Online] http://en.wikipedia.org/wiki/Binary_image.

15. [Online] http://en.wikipedia.org/wiki/Canny_edge_detector.

16. Snakes: Active contour models. MICHAEL. KASS, ANDREW WITKIN, and DEMETRI

TERZOPOULOS. 1988 йил, International Journal of Computer Vision.

17. Active Contour External Force Using Vector. Bing Li, Scott Acton. 8, 2007 йил, IEEE Transactions on Image Processing, Vol. 16. 
18. A new and Simplified Method for Coronary Graft Imaging During CABG. Fraser D Rubens, Marc Ruel, Stephen E Fremes. 2002 йил, The Heart Surgery Forum, Vol. 5.

19. Lucia M A Crane, George Themelis. Intraoperative Multispectral Fluorescence Imaging for the detection of the Sentinel Lymph Node in Cervical Cancer: A Novel Concept. s.1. : Molecular Imaging and Biology, 2010.

20. An Operational Near-Infrared Fluorescence Imaging System Prototype for Large Animal Surgery. De Grand, A.M., Frangioni, J.V. 6, s.l. : Adenine Press, 2003 йил December, Technology in Cancer Research \& Treatment, Vol. 2.

21. Sentinel lymph node mapping of invasive urinary bladder cancer in animal models using invisible light. Knapp, D.W., Adams, L.G., Degrand, A.M., Niles, J.D., Ramos-Vara, J.A., Weil, A.B., O'Donnell, M.A., Lucroy, M.D., Frangioni, J.V. 6, 2007 йил December, European Urology, Vol. 52, pp. 1700-1708.

22. Near-infrared fluorescence imaging of liver metastases in rats using indocyanine green. van der Vorst, J.R., Hutteman, M., Mieog, J.S., de Rooij, K.E., Kaijzel, E.L., Löwik, C.W., Putter, H., Kuppen, P.J., Frangioni, J.V., van de Velde, C.J., Vahrmeijer, A.L. 2, 2012 йил Мау, The Journal of Surgical Research, Vol. 174, pp. 266-271.

23. Image-guided sentinel lymph node mapping and nanotechnology-based nodal treatment in lung cancer using invisible near-infrared fluorescent light. Khullar, O., Frangioni, J.V., Grinstaff, M., Colson, Y.L. 4, 2009 йил, Seminars in Thoracic and Cardiovascular Surgery, Vol. 21, pp. 309-315.

24. Clinical translation of ex vivo sentinel lymph node mapping for colorectal cancer using invisible nearinfrared fluorescence light. Hutteman, M., Choi, H.S., Mieog, J.S., van der Vorst, J.R., Ashitate, Y., Kuppen, P.J., van Groningen, M.C., Löwik, C.W., Smit, V.T., van de Velde, C.J., Frangioni, J.V., Vahrmeijer, A.L. 4, 2011 йил April, Annals of Surgical Oncology, Vol. 18, pp. 1006-1014.

25. The FLARE Intraoperative Near-Infrared Fluorescence Imaging System: A First-in-Human Clinical Trial in Breast Cancer Sentinel Lymph Node Mapping. Troyan, S.L., Kianzad, V.,Gibbs-Strauss, S.L., Gioux, S., Matsui, A., Oketokoun, R., Ngo, L., Khamene, A., Azar, F., Frangioni, J.V. 2009 йил, Annals of Surgical Oncology, Vol. 16, pp. 2943-2952.

26. Toward Optimization of Imaging System and Lymphatic Tracer for Near-Infrared Fluorescent Sentinel Lymph Node Mapping in Breast Cancer. Mieog, J.S.D., Troyan, S.L., Hutteman, M., Donohoe, K.J., van der Vorst, J.R., Stockdale, A., Liefers, G., Choi, H.S., Putter, H., Gioux, S., Kuppen, P.J.K., Ashitate, Y., Löwik, C.W.G.M., Oketokoun, R., Ngo, L.H., Frangioni, J.V., Vahrmeijer, A.L. 9, 2011 йил, Annals of Surgical Oncology, Vol. 18, pp. 2483-2491.

27. Near-infrared fluorescence sentinel lymph node mapping of the oral cavity in head and neck cancer patients. van der Vorst, J.R., Schaafsma, B.E., Verbeek, F.P., Keereweer, S., Jansen, J.C., van der Velden, L.A., Langeveld, A.P., Hutteman, M., Löwik, C.W., van de Velde, C.J., Frangioni, J.V., Vahrmeijer, A.L. 1, 2013 йил January, Oral Oncology, Vol. 49, pp. 15-19.

28. Randomized comparison of near-infrared fluorescence lymphatic tracers for sentinel lymph node mapping of cervical cancer. Schaafsma, B.E., van der Vorst, J.R., Gaarenstroom, K.N., Peters, A.A., Verbeek, F.P., de Kroon, C.D., Trimbos, J.B., van Poelgeest, M.I., Frangioni, J.V., van de Velde, C.J., Vahrmeijer, A.L. 1, 2012 йил October, Gynecologic Oncology, Vol. 127, pp. 126-130. 
29. New device for intraoperative graft assessment: HyperEye charge-coupled device camera system.

Handa, T., Katare, R.G., Nishimori, H., Wariishi, S.,Fukutomi, T., Yamamoto, M., Sasguri, S., Sato, T. 2010 йил, General Thoracic and Cardiovascular Surgery, Vol. 58, pp. 68-77.

30. Feasibility of ICG Fluorescence-Guided Sentinel Node Biopsy in animal Models using the HyperEye Medical System. Yamauchi, K., Nagafuji, H., Nakamura, T., Sato, T., Kohno, N. 2011 йил, Annals of Surgical Oncology, Vol. 18, pp. 2042-2047.

31. A New and Simplified Method for Coronary and Graft Imaging During CABG. Rubens, F.D., Ruel, M., Fremens, S.E. 2, 2002 йил, The Heart Surgery Forum, Vol. 5, pp. 141-144.

32. Novadaq SPY: intraoperative Quality Assessment in Off-Pump Coronary Artery Bypass Grafting. Reuthebuch, O., Häussler, A., Genoni, M., Tavakoli, R., Odavic, D., Kadner, A., Turina, M. 2, 2004 йил February, Chest, Vol. 125, pp. 418-424.

33. An intraoperative fluorescent imaging system in organ transplantation. Sekijima, M., Tojimbara, T., Sato, S., Nakamura, M., Kawase, T., Kai, K., Urashima, Y., Nakajima, I., Fuchinoue, S., Teraoka, S. 7, 2004 йил September, Transplantation Proceedings, Vol. 36, pp. 2188-2190.

34. Novadaq SpyTM Intraoperative Imaging System - Current Status. Vogt, P.R., Bauer, E.P., Graves, K. 2003 йил, The Journal of Thoracic and Cardiovascular Surgery, Vol. 51, pp. 49-51.

35. Optimization of Coded Aperture Radioscintigraphy for Sentinel Lymph Node Mapping. Hirofumi Fuji, John D Idoine, Sylvain Gioux, Roberto Accorsi, David R Slochower, Richard C Lanza, John v Frangioni. 2011 йил, Molecular Imaging and Biology.

36. High-energy-resolution scintillator: Ce3+ activated LaBr3. van Loef, E.V.D., Dorenbos, P., van Eijk, C.W.E., Krämer, K.W., Güdel, H.U. 10, 2001 йил, Applied Physics Letters, Vol. 79, pp. 1573-1575.

37. Newly developed semiconductor detectors by. Yamamoto, K., Yamamura, K., Sato, K., Kamakura, S., Ota, T., Suzuki, H., Ohsuka, S. Kobe, Japan : International School for Advanced Studies, Trieste, Italy, 2007. International workshop on new photon-detector.

38. Silicon photomultiplier and its possible applications. Buzhan, P., Dolgoshein, B., Filatov, L., Ilyin, A., Kantzerov, V., Kaplin, V., Karakash, A., Kayumov, F., Klemin, S., Popova, E., Smirnov, S. 2003 йил, Nuclear Instruments and Methods in Physics Research A, Vol. 504, pp. 48-52.

39. Kross, B.J., McKisson, J., Stolin, A., Weisenberger, A.G., Zorn, C. Collimator with attachment mechanism and system. 8217359 USA, 2012 йил 10-07.

40. Light-absorbing properties, stability, and spectral stabilization of indocyanine green. Landsman, M.L.J., Kwant, G., Mook, G.A., Zijlstra, W.G. 1976 йил, Journal of Applied Physiology, Vol. 40, pp. 575-583.

41. Near-infrared optical imaging of protease activity for tumor detection. Mahmood, U., Tang, C.H., Bogdanov, A., Weissleder, R. 1999 йил, Radiology, Vol. 213, pp. 866-870.

42. Fluorescence-enhanced, near infrared diagnostic imaging with contrast agents. Sevick-Muraca, E.M., Houston, J.P., Gurfinkel, М. 5, 2002 йил October, Current opinion in chemical biology, Vol. 6, pp. 642650 . 
43. Fluorescence imaging with near-infrared light: new technological advances that enable in vivo molecular imaging. Ntziachristos, V., Bremer, C., Weissleder R. 1, 2003 йил January, European radiology, Vol. 13, pp. 195-208.

44. Marubeni America Corporation. [Online] [Cited: 2012 йил 20-August.] http://www.techled.com/data/Illuminator_Radiation.pdf.

45. Real-time intraoperative fluorescence imaging system using light-absorption correction. Themelis, G., Yoo, J.S., Soh, K., Schulz, R., Ntziachristos, V. 6, 2009 йил November/December, Journal of Biomedical Optics, Vol. 14, p. 064012.

46. ntraoperative Multispectral Fluorescence Imaging for the Detection of the Sentinel Lymph Node in Cervical Cancer: A Novel Concept. Crane, L.M.A., Themelis, G.,Pleijhuis, R.G., Harlaar, N.J., Sarantopoulos, A., Arts, H.J.G., van der Zee, A.G.J., Ntziachristos, V., van Dam, G.M. 2011 йил, Molecular Imaging and Biology, Vol. 13, pp. 1043-1049.

47. Near-infrared fluorescence (NIRF) imaging in breast-conserving surgery: Assessing intraoperative techniques in tissue-simulating breast phantoms. Pleijhuis, R.G., Langhout, G.C., Helfrich, W., Themelis, G., Sarantopoulos, A., Crane, L.M., Harlaar, N.J., de Jong, J.S., Ntziachristos, V., van Dam, G.M. 2011 йил, European Journal of Surgical Oncology, Vol. 37, pp. 32-39.

48. Multiplex techniques for frequency-domain photon migration imaging. Reynolds, J.S., Troy, T.L., Sevick-Muraca, E.M. 1997 йил, Biotechnology Progress, Vol. 13, pp. 669-680.

49. Imaging of lymph flow in breast cancer patients after microdose administration of a near-infrared fluorophore: feasibility study. Sevick-Muraca, E.M., Sharma, R., Rasmussen, J.C., Marshall, M.V., Wendt, J.A., Pham, H.Q., Bonefas, E., Houston, J.P., Sampath, L., Adams, K.E., Blanchard, D.K., Fisher, R.E., Chiang, S.B., Elledge, R., Mawad, M.E. 3, 2008 йил March, Radiology, Vol. 246, pp. 734741.

50. Fluorescence Navigation with Indocyanine Green for Detecting Sentinel Lymph Nodes in Breast Cancer. Kitai, T., Inomoto, T., Miwa, M., Shikuyama, T. 3, 2005 йил, Breast Cancer, Vol. 12, pp. 211 215 .

51. Intraoperative identification of sentinel lymph nodes by near-infrared fluorescence imaging in patients with breast cancer. Tagaya, N., Yamazaki, R., Nakagawa, A., Abe, A., Hamada, K., Kubota, K., Oyama, Т. 2008 йил, The American Journal of Surgery, Vol. 195, pp. 850-853.

52. Evaluation of Breast Lymphatic Pathways with Indocyanine Green Fluorescence Imaging in Patients with Breast Cancer. Ogasawara, Y., Ikeda, H., Takahashi, M., Kawasaki, K., Doihara, H. 2008 йил, World Journal of Surgery, Vol. 32, pp. 1924-1929.

53. The usefulness of photodynamic eye for sentinel ymph node identification in patients with cervical cancer. Furukawa, M., Oi, H., Yoshida, S., Shigetomi, H., Kanayama, S., Kobayashi, H. 96, 2010 йил, Tumori, pp. 936-940.

54. An Experimental Study to Evaluate the Fluobeam 800 Imaging System for Fluorescence-Guided Lymphatic Imaging and Sentinel Node Biopsy. Hirche, C., Engel, H., Kolios, L., Cognie, J., Hünerbein, M., Lehnhardt, M., Kremer, T. 2012 йил December, Surgical Innovation, pp. 1-8. doi:

$10.1177 / 1553350612468962$. 
55. ICG fluorescence-guided sentinel node biopsy for axillary nodal staging in breast cancer. Hirche, $\mathbf{C}$., Murawa, D., Mohr, Z., Kneif, S., Hunerbein, М. 2010 йил, Breast Cancer Research and Treatmant, Vol. 121, pp. 373-378.

56. Sentinel lymph node biopsy in breast cancer guided by indocyanine green fluorescence. Murawa, D., Hirche, C., Dresel, S., Hunerbein, М. 2009 йил, British Journal of Surgery, Vol. 96, pp. 1289-1294.

57. Laser-induced fluorescence of indocyanine green: plastic surgical applications. Holm, C., Mayr, M., Tegeler, J., Becker, A., Pfeiffer, A., Muhlbauer W. 2003 йил, European Journal of Plastic Surgery, Vol. 26, pp. 19-25.

58. Validation of IC-VIEW fluorescence videography in a rabbit model of mesenteric ischaemia and reperfusion. Toens, C., Krones, C.J., Blum, U., Fernandez, V., Grommes, J., Hoelzl, F., Stumpf, M., Klinge, U., Schumpelick, V. 4, 2006 йил May, International Journal of Colorectal Disease, Vol. 21, pp. $332-338$.

59. Nanocolloidal albumin-IRDye 800CW: a near-infrared fluorescent tracer with optimal retention in the sentinel lymph node. Heuveling, D.A., Visser, G.W., de Groot, M., de Boer, J.F., Baclayon, M., Roos, W.H., Wuite, G.J., Leemans, C.R., de Bree, R., van Dongen, G.A. 7, 2012 йил July, European Journal of Nuclear Medicine and Molecular Imaging, Vol. 39, pp. 1161-1168. 